\section{Epidemiology of stroke}

\author{
1 Epidemiology of stroke
}

STROKE AND PLASMA MARKERS OF MILK FAT INTAKE A PROSPECTIVE NESTED CASE-CONTROL STUDY

E. Warensjö ${ }^{1}$, A. Smedman ${ }^{1}$, B. Stegmayr ${ }^{2}$, G. Hallmans ${ }^{3}$, L. Weinehall ${ }^{3}$, B. Vessby ${ }^{1}$, I. Johansson ${ }^{4}$

${ }^{1}$ Department of Public Health and Caring Sciences, Clinical nutrition and metabolism, Uppsala University, Uppsala, Sweden; ${ }^{2}$ Department of Public Health and Clinical Medicine, Umeå University and National Board of Heath and Welfare (STOCKHOLM), Umeå, Sweden; ${ }^{3}$ Department of Public Health and Clinical Medicine, Umeå University, Umeå, Sweden; ${ }^{4}$ Department of Odontology, Umeå University, Umeå, Sweden

Background: Dairy products are high in saturated fat and are traditionally considered a risk factor for vascular diseases. The fatty acids 15:0 and 17:0 of plasma lipids are considered as reliable and objective biomarkers of milk fat intake. The aim of the present study was to evaluate the risk of a first-ever stroke event in Northern Sweden in relation to the milk fat biomarkers in plasma lipids (cholesteryl esters and phospholipids)

Methods: A prospective case-control study was nested within two population based health surveys in Northern Sweden. Among 129 stroke cases and 257 matched controls, plasma samples for fatty acid analyses were available in 108 cases and 216 control subjects. Proportions of 15:0 and 17:0 in plasma lipids, weight, height, blood lipids, blood pressures, and lifestyle data were employed in conditional logistic regression modelling.

Results: The proportions of fatty acids 17:0 and 15:0+17:0 of total plasma phospholipids were significantly higher in female controls than cases, but not in men. The standardised odds ratio $(95 \% \mathrm{CI})$ in women to have a stroke was $0.41(0.24-0.69)$ and 0.48 (95\% CI $0.29-0.79)$ for $17: 0$ and $15: 0+17: 0$ of plasma phospholipids, respectively. Adjustment for traditional cardiovascular risk factors, physical activity and diet (fish, fruits and berries, vegetables and alcohol) had marginal effects on the odds ratios. A similar, but non-significant, trend was seen in men.

Conclusions: It is hypothesised that dairy product or milk fat intake may be inversely related to the risk of a first event of stroke. The intriguing results of this study should be interpreted with caution and the results should be followed up in future studies.

\section{Epidemiology of stroke}

LUNG FUNCTION PREDICTS FATAL STROKE IRRESPECTIVELY OF BODY MASS AND SMOKING HABITS. A 42 YEAR FOLLOW-UP STUDY IN A GENERAL POPULATION

A.K. Gulsvik ${ }^{1}$, M. Mowé ${ }^{2}$, D.S. Thelle ${ }^{3}$, T.B. Wyller ${ }^{2}$

${ }^{1}$ Department of Geriatric Medicine, Ullevaal University Hospital, University of Oslo, Oslo, Norway; ${ }^{2}$ Department of Geriatric Medicine, Aker University Hospital, University of Oslo, Oslo, Norway; ${ }^{3}$ Department of Biostatistics, University of Oslo, Oslo, Norway

Background: During the major part of adult life the body mass index (BMI) tends to increase, the lung function declines and the risk of cerebral stroke increases. We wanted to study the assosiation between BMI and lung function on one side and the outcome of letal stroke.

Methods: A random sample of 6811 Norwegians was invited to a longitudinal cohort study in 1964. 5653 individuals (84\%) attended. The mean age was 47.5 years; range 22-75 years. BMI $\left(\mathrm{kg} / \mathrm{m}^{2}\right)$ was calculated from standardized measurements of body height and weight and divided in four groups ( $<22.0,22.0-24.9$, $25.0-27.9, \geq 28.0$ ). Forced expiratory volume after one second (FEV1) (1) was measured spirometrically and the percentage of the reference value was calculated (FEV1\%). Cerebral stroke as cause of death was registered in the ICD 6-10 code system at National Statistics of Norway. The mean follow up time was 28 years.

Results: 543 individuals died from cerebral stroke during follow up. In women the lowest risk of fatal stroke was in the group with BMI less than $22 \mathrm{~kg} / \mathrm{m}^{2}$ (Cox proportional hazard ratio (HR) $0.60,95 \%$ confidence interval (CI) [0.40-0.91], $\mathrm{p}=$ 0.016 ), compared to the group with BMI $22.0-24.9 \mathrm{~kg} / \mathrm{m}^{2}$ (reference), and there was a statistically nonsignificant trend towards increasing risk with increasing BMI. In men no significant BMI-stroke-associations were found.

Lung function (FEV1\%) was significantly associated to the risk of fatal stroke for both genders. A ten percent decline in FEV1\% was associated to an increased risk of fatal stroke for both genders (HR 1.11, 95\% CI [1.03-1.20], $\mathrm{p}=0.005$ and 1.11 , $95 \%$ CI [1.05-1.19], $\mathrm{p}=1.001$, for men and women respectively) after adjustments to smoking habits. BMI did not significantly influence the FEV1-stroke association.
Conclusion: BMI is a weak predictor of fatal cerebral stroke in women. Lung function is a significant risk factor for cerebral stroke later in life independently of BMI and smoking.

\section{Epidemiology of stroke}

MORTALITY AND PREDICTORS OF DEATH ONE MONTH AND THREE YEARS AFTER ISCHEMIC STROKE: DATA FROM THE FIRST NATIONAL ACUTE STROKE ISRAELI SURVEY (NASIS 2004)

S. Koton ${ }^{1}$, D. Tanne ${ }^{2}$, M.S. Green ${ }^{3}$, N.M. Bornstein ${ }^{4}$

${ }^{1}$ Stanley Steyer School of Health Professions, Sackler Faculty of Medicine, Tel-Aviv University, Tel Aviv, Israel; ${ }^{2}$ Stroke Center, Department of Neurology and Sagol Neuroscience Center, Chaim Sheba Medical Center, Tel Hashomer, Israel; ${ }^{3}$ School of Public Health, University of Haifa, Haifa, Israel; ${ }^{4}$ Neurology Department and Stroke Unit, Tel Aviv Sourasky Medical Center, Tel Aviv, Israel

Background: Predictors of short-term versus long-term mortality after ischemic stroke (IS) may differ. Based on a national survey, we present the rates of mortality after acute IS during the first 3-years after stroke and the major predictors of short-term (1-month) versus long-term (3-years) mortality.

Methods: In NASIS 2004 data were collected on all hospitalized acute stroke patients in Israel during a two-month period. Mortality rates for IS were assessed at 1-month and 3-years by means of matching patients' files with national mortality data. Determinants of death were evaluated using the Cox proportional hazard model at 1-month and 3-years.

Results: A total of 1,558 IS patients were included. Survival data were complete for over $99 \%$ of patients. Cumulative rates of mortality were $9.1 \%$ at 1 -month and $34.1 \%$ at 3-years. At 1-month, decreased level of consciousness (HR 3.5, 95\% CI 2.2-5.7), total anterior circulation infarction (TACI) $(4.3,1.6-11.5)$, temperature on admission $(1.4,1.1-1.8$ per $10 \mathrm{C})$, age (1.04, 1.02-1.106 per year) and glucose levels on admission $(1.004,1.001-1.006$ per $1 \mathrm{mg} / \mathrm{dL})$ were positively associated with death. Death risk increased with stroke severity (by NIHSS, $\mathrm{p}<0.05$ ). Predictors of death at 1-month predicted also death at 3-years, however, history of dementia (HR 1.5, 95\% CI 1.1-1.9), diabetes $(1.4,1.1-1.7)$, peripheral artery disease (1.8, $1.3-2.4)$, chronic heart failure $(1.5,1.2-1.9)$ and angina pectoris $(1.3,1.02-1.7)$ were additional determinants of long-term mortality.

Conclusions: Over one-third of patients do not survive three years after an IS. Age and markers of stroke severity predicted both short and long-term mortality, whereas comorbidities and variables associated with atherosclerotic vascular disease were important additional determinants of long-term mortality after IS.

\section{Epidemiology of stroke}

\section{MARKED REDUCTION IN CARDIOVASCULAR DEATHS IN STROKE PATIENTS. A NORTHERN SWEDEN MONICA STROKE REGISTRY STUDY E. Uneus, M. Eriksson, P.-G. Wiklund}

Dept of Medicine, Umeå University, Umeå, Sweden

Background: Decreased mortality, and stroke severity, in stroke patients has been reported from several countries. The cause of death in stroke patients is less well studied and the aim of this study was to investigate changes in the patterns of mortality and causes of death. The Northern Sweden MONICA Stroke registry records stroke events in the north of Sweden since 1985. It has been shown that the registry captures $95 \%$ of the stroke cases in the region.

Methods: First ever stroke events in the ages 24 to 74, between January 1, 1985 and December 31, 2003, were identified in the stroke registry. WHO stroke criteria was applied. Subarachnoidal hemorrhages were excluded. Four time-periods were compared in the analyses (1985-1989, 1990-1994, 1995-1999, 2000-2003). Death, and cause of death, at 7, 28, 90, 365 and 730 days were analyzed.

Results: There were 11594 cases identified. Mean age 64,5 years, 61\% men, 39\% women, with no significant changes over time. Mortality declined significantly over time at all time points, comparing the first to the fourth time-period, at 2 years $(29,3 \%$ to $18,0 \%)$, at 7 days $(10.3 \%$ to $7.5 \%)$. Death at 2 years in stroke-related and other cardiovascular causes was reduced, 12.3 to $8.4 \%$ and 9.2 to $4,6 \%$ respectively. No reduction in deaths related to infection, malignancy or other causes was found, nor was any sex-differences observed.

Subsequent analyses of the distribution of deaths over the different periods: 0-7,828, 29-90, 91-365 and 366-730 days, showed a consistent trend of reduction in later phases but a higher proportion of deaths was occurring early (0-7) in 2000-2003 compared to in 1985-1989, p 0.02 .

Conclusion: Mortality in stroke patients has declined between 1985 and 2003 in the north of Sweden. The reduction is seen both early and late after stroke and can be ascribed to lower cardiovascular death rates. The less pronounced reduction in the acute phase may have implications for the further development of stroke care. 
5 Epidemiology of stroke

COGNITIVE FUNCTION IN ELDERLY MEN IS A PREDICTOR OF ISCHEMIC STROKE

B. Wiberg ${ }^{1}$, L. Lind ${ }^{2}$, L. Kilander ${ }^{1}$, B. Zethelius ${ }^{1}$, J. Sundström ${ }^{2}$

${ }^{1}$ Department of Public Health and Caring Sciences/Geriatrics, Uppsala,

Sweden; ${ }^{2}$ Department of Medical Sciences, Uppsala, Sweden

Background: Our aim was to study the predictive power of cognitive function for later incidence of fatal or non-fatal stroke or transient ischemic attacks (TIA) and stroke subtypes. To measure the cognitive function we used three different tests.

Methods: The study is based on the Uppsala longitudinal Study of Adult Men cohort. The examinations were performed at age 70 years. The cognitive tests used were the Mini Mental State Examination (MMSE) and Trail Making Test (TMT) A and B.

Results: In 930 men, free from stroke at baseline, 166 developed stroke or TIA during a follow-up of up to 13 years using registry data obtained from the Swedish Hospital Discharge Record (SHDR) and the Cause-of death Register (CDR). In univariate Cox proportional hazards analyses, a 1-standard deviation increase in result TMT B was associated with a higher risk for brain infarction (HR 1.86, 95\% CI 1.14-3.04, $\mathrm{n}=105$ ) but not for the total group stroke/TIA (HR 1.24 $95 \%$ 0.84-1.83) or the subgroup of intra cerebral haemorrhages (HR 1.40, 95\% $0.50-3.91$ ). The predictive value of TMT B was still significant after adjustment for education level and traditional risk factors, such as blood pressure.

Conclusion: Cognitive function in elderly men, measured by Trail Making Test $\mathrm{B}$, predicted subsequent brain infarction independently of education and traditional risk factors in a community-based sample of elderly men.

\section{Epidemiology of stroke}

INCIDENCE AND CASE FATALITY OF SUBARACHNOID HEMORRHAGE IN NORTHERN GREECE: THE EVROS REGISTRY OF SUBARACHNOID HEMORRHAGE (EVRO-SAH)

K. Vadikolias, G. Tsivgoulis, I. Heliopoulos, M. Papaioakim, C. Aggelopoulou, A. Serdari, T. Birbilis, C. Piperidou

Department of Neurology, University Hospital of Alexandroupolis, Democritus University of Thrace, Alexandroupolis, Greece

Background: Epidemiological data on subarachnoid hemorrhage (SAH) incidence and case fatality rates are scarce in the South-Eastern Mediterranean region. We conducted a population-based study in Evros province, located in north eastern Greece, to determine SAH incidence over a 5-year period.

Methods: Evros province has a well-defined, largely-homogeneous population with healthcare organized around a single tertiary-care University Hospital. We organized a prospective computerized registry of permanent Evros residents admitted or transferred to our hospital with a diagnosis of SAH. Standard WHO definitions and overlapping case-finding methods were used to identify all cases of first-ever in a lifetime $\mathrm{SAH}$ in all age-groups, occurring during the study period. The diagnosis was confirmed by CT-scan in all hospitalized cases. Sudden deaths attributable to SAH were systematically recorded province-wide by our Forensic Department and included into the study.

Results: During the 5-year period 51 cases of SAH were recorded (28 men, 23 women; mean age 59 \pm 17 years). The crude annual incidence-rates were $8.3 \% / 100.000$ persons (95\%CI:5.5\%-\%12.0\%) for men, $7.5 \% / 100.000(4.8 \%-$ $11.3 \%$ ) for women and $7.9 \% / 100.000(5.9 \%-10.4 \%)$ for all subjects. The standardized incidence rates for groups aged 45-84 years to the European population were $9.3 \% / 100.000(5.8 \%-12.8 \%)$ for men, $6.5 \% / 100.000(3.7 \%-9.4 \%)$ for women and $7.9 \% / 100.000(5.7 \%-10.2 \%)$ overall. The 28 -day case fatality rates for men, women and all subjects were $36 \%(21 \%-54 \%), 35 \%(19 \%-55 \%)$ and $35 \%(24 \%-49 \%)$ respectively

Conclusions: The incidence and case fatality rate of SAH hemorrhage in Greece appear to be similar to other industrialized countries. No gender-differences in SAH incidence and case-fatality rate were documented.

\section{Epidemiology of stroke}

\section{THE LUDWIGSHAFEN STROKE STUDY (LUSST) CASE FATALITY RATES} FOR FIRST-EVER STROKE (FES)

S.B. Rose, F. Palm, B. Bode, C. Urbanek, F. Buggle, A.J. Grau

Department of Neurology, Klinikum Ludwigshafen, Ludwigshafen am Rhein, Germany

Background and purpose: Improvements in stroke care can be expected to lead to lower mortality after stroke. Population-based stroke registries are required to obtain reliable data on stroke mortality. Here we report results from a populationbased stroke registry from Ludwigshafen a.Rh., an industrial city in Southwestern Germany.

Methods: The Ludwigshafen Stroke Study (LuSSt) is a population-based stroke registry in the city of Ludwigshafen (about 167.000 residents), RhinelandPalatine, Germany. Starting January 2006 multiple overlapping methods of caseascertainment were used. Follow-up was performed at 28 days, 3 and 12 months.

Results: In 2006347 patients suffered a first ever ischemic stroke and 34 a first ever intracerebral hemorrhage (Total: 381 ). Follow-up could be performed in 362 $(95,0 \%)$ patients at day 28 and after 3 months, respectively and in $359(94,2 \%)$ patients after 12 months. Overall crude case fatality rates were $11,9 \%(95 \% \mathrm{CI}$ $8,9-15,6)$ at day $28,16,3 \%(95 \% \mathrm{CI}: 12,9-20,5)$ at 3 months and $23,4 \%(95 \% \mathrm{CI}$ : $19,3-20,0)$ at 12 months. Case fatality rates for ischemic stroke was $10,0 \%$ at day $30,13,9 \%$ at 3 months and $21,7 \%$ at 12 months. Respective rates for intracerebral hemorrhage were $28,1 \%$ at day 28 , and $40,6 \%$ both at 3 and 12 months.

Conclusion: Case fatality rates after ischemic and particularly after hemorrhagic stroke are still high, however, our present rates are lower than those from previous European population based stroke registries. This could reflect recent improvements in stroke care, however, comparisons between different countries and different times have to be viewed with caution and our data are only based on the analysis of one year.

\section{Epidemiology of stroke}

\section{THE DETERMINANTS OF HEALTH-RELATED QUALITY OF LIFE AFTER} STROKE IN CHINA. RESULTS OF THE CHINAQUEST (QUALITY EVALUATION OF STROKE CARE AND TREATMENT) STUDY

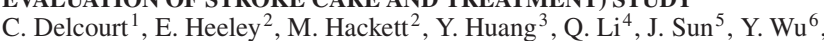
J. Wang ${ }^{7}$, C. Anderson ${ }^{8}$

${ }^{1}$ The George Institute for International Health-Royal Prince Alfred Hospital, Sydney, Australia; ${ }^{2}$ The George Institute for International Health-University of Sydney, Sydney, Australia; ${ }^{3}$ Peking University First Hospital, Beijing, China; ${ }^{4}$ The George Institute for International Health, Sydney, Australia; ${ }^{5}$ The George Institute for International Health, Beijing, China; ${ }^{6}$ The George Institute for International Health-Peking University Health Science Centre, Beijing, Australia: ${ }^{7}$ Centre for Epidemiological Studies and Clinical Trials-Ruijin Hospital-Shanghai Institute for Hypertension, Shanghai, China; ${ }^{8}$ The George Institute for International Health-Royal Prince Alfred Hospital-University of Sydney, Sydney, Australia

Background: Despite the enormous burden of stroke, there is limited information on health-related quality of life (HRQoL) of patients with stroke in China. The ChinaQUEST study aimed to determine various outcomes from stroke over 12 months.

Methods: The ChinaQUEST study, a prospective study in 4283 patients from 62 hospitals across all provinces in China, used a Chinese 35 -item quality of life scale questionnaire (QoL35), developed from the 100-Item World-Health Organization QoL Instrument and the 36-Item Outcomes Study Short Form Health Status Survey, to assess HRQoL among 12-month survivors. The major difference between QoL 35 and other HRQoL questionnaires is a greater emphasis on family relationships. Proxy responses were used in patients unable to directly complete the QoL 35 . Multilevel modeling with random intercept for centre level and fixed effect for covariates was used to develop a predictive model of low HRQoL.

Results: 4283 patients (59.6\% proxy-responders, $40.4 \%$ self-responders) were assessed for HRQoL at 12 months. Baseline factors predicting low (below median) HRQoL scores in proxy-responders were older age (odds ratio [OR] 1.03; 95\% CI $1.02-1.05)$, being dependent at baseline $(1.45 ; 1.06-1.99)$, having diabetes $(1.37$; $1.03-1.82)$, prior stroke/TIA $(1.56 ; 1.25-1.96)$, having a more extensive stroke (OCSP classification, PACS vs TACS $0.66 ; 0.45-0.97$ ), being disabled at discharge $(4.73 ; 3.77-5.95)$ and having a severe stroke defined by a GCS $<8$ (GCS $\geq 8$ vs $<80.50 ; 0.29-0.88)$. Self-responders had social and medical factors included in the model: being older $(1.02 ; 1.01-1.03)$, having a lower income $(>19,000$ $(>2782$ US $) v s<10000 C N Y(<1464 U S), 0.48 ; 0.33-0.71)$, having diabetes $(1.44$; $1.03-2.02)$ and being disabled at discharge $(3.44 ; 2.54-4.66)$.

Conclusion: As shown elsewhere, HRQoL after stroke is mainly determined by the level of disability in China. However, level of income and co-morbid vascular risk factors are also important in this population. 
9 Epidemiology of stroke

INFLAMMATORY MARKERS AND POOR OUTCOME AFTER STROKE: A PROSPECTIVE COHORT STUDY AND META-ANALYSIS

W.N. Whiteley ${ }^{1}$, C. Jackson ${ }^{1}$, S. Lewis ${ }^{1}$, G. Lowe ${ }^{2}$, A. Rumley ${ }^{2}$, P. Sandercock ${ }^{1}$, J. Wardlaw ${ }^{1}$, M. Dennis ${ }^{1}$, C. Sudlow ${ }^{1}$

${ }^{1}$ Division of Clinical Neurosciences, Western General Hospital, University of Edinburgh, Edinburgh, United Kingdom; ${ }^{2}$ Division of Cardiovascular and Medical Sciences, Royal Infirmary, University of Glasgow, Glasgow, United Kingdom

Background: We sought to determine whether: (a) markers of acute inflammation (white cell count, glucose, interleukin 6 (IL-6), C-reactive protein (CRP) and fibrinogen) are associated with poor outcome after all stroke and (b) the addition of markers to previously validated prognostic models improves prediction of poor outcome.

Methods: We prospectively recruited stroke patients from an emergency department, medical, stroke and neurology wards and a neurovascular clinic. Clinicians recorded data at the time of assessment and drew blood for measurement of inflammatory markers. Patients were followed up at 6 months for death and poor outcome ( $>2$ modified Rankin Scale).

Results: We recruited 844 patients with blood marker data; at 6 months, mortality data was available in $844(100 \%)$ and functional outcome in $750(89 \%)$. After adjustment for age, and whether the patient: lived alone, was independent of activities of daily living, orientated, able to lift arms off the bed and able to walk, the OR for the association of markers and death (comparing the upper and the lower tertile) after stroke were: IL-6, 5.7 (95\% CI:1.9 to 17.1); CRP 3.5 (95\% CI:1.4 to 9.1); fibrinogen, 1.2 (95\% CI:0.6 to 2.6); white cell count 1.5 (95\% CI:1.4 to 5.5 ) and glucose 1.8 (95\% CI:1.6-7.4). The OR for the association of markers and poor outcome (comparing the upper and the lower tertile) were: IL-6 3.1 (95\% CI:1.9 to 5.0); CRP 1.9 (95\% CI 1.2 to 3.1); fibrinogen 1.5 (95\% CI:1.0 to 2.36), white cell count 2.1 (95\% CI 1.3 to 3.4 ) and glucose 1.3 (95\% CI 0.8 to 2.1 ). However, the addition of marker levels to validated prognostic models did not materially improve model discrimination, calibration or reclassification for prediction of poor outcome. Conclusions: Raised levels of markers of the acute inflammatory response after stroke are associated with poor outcome. However, the addition of these markers to previously validated stroke prognostic models did not improve the prediction of poor outcome.

\section{Epidemiology of stroke}

\section{SINGLE QUESTIONS TO SCREEN FOR DEPRESSION MISS IMPORTANT NEGATIVE COGNITIONS \\ M.L. Hackett ${ }^{1}$, K.M. Hill ${ }^{2}$, J. Hewison ${ }^{2}$, C.S. Anderson ${ }^{1}$, A.O. House ${ }^{2}$ \\ ${ }^{1}$ The George Institute for International Health, Sydney, Australia; ${ }^{2}$ Leeds Institute of Health Sciences, Leeds, United Kingdom}

Background: There has been an increase in the use of single questions to screen for depression in the physically-ill. We explored the clinical applicability of this approach in two independently conducted stroke studies with similar methods.

Methods: The Auckland Regional Community Stroke (ARCOS) study was a population-based stroke incidence study conducted in Auckland, New Zealand over a 12-month period in 2002-2003. The Stroke Outcomes Study (SOS2) was a prospective hospital cohort study conducted in Leeds and Bradford, United Kingdom over a 33-month period in 2002-2005. Symptoms of depression were assessed at six months in ARCOS using a single simple question "do you often feel sad and depressed?" and the 28-item General Health Questionnaire (GHQ-28), and in SOS2 using the GHQ-28 and a single question about depressed mood taken from the Present State Examination (PSE).

Results: Data were available from 770 ARCOS and 492 SOS2 patients at six months. ARCOS and SOS2 patients were similar in age, sex and marital status. More ARCOS participants were completely independent in activities for daily living, while more SOS2 participants met the criteria for depression. A significant proportion (up to $16 \%$ ) of people who did not meet the single question criteria for depression reported important suicidal and depression-related negative cognitions of clinical concern.

Conclusions: Important negative cognitions may be missed when single questions are used to screen for depression after stroke. Brief screening methods are not an adequate substitute for a sensitive exploration of the psychological impact of stroke on the survivor.
11 Epidemiology of stroke

\section{STROKE AWARENESS IN DENMARK}

T. Truelsen, L.H. Krarup

Department of Neurology, Bispebjerg university hospital, Copenhagen, Denmark

Background: The knowledge in the general population about major stroke symptoms and risk factors is essential for fast treatment, and for improving stroke prevention. This is the first-ever study about stroke knowledge in a large Danish population.

Methods: A total of 3,520 subjects were invited to participate in the study using a web-based, (closed-) questionnaire. The recruitment was stratified by region, age, and gender to represent the composition of the general Danish population aged $25+$ years. Enrolment was competitive with a pre-defined target of 800 responses. Subjects were asked to rank the 4 most important acute stroke symptoms (sudden onset of hemiparesis, facial palsy, aphasia, and disturbances of sensation), and the 3 most important risk factors for stroke (hypertension, diabetes, tobacco smoking). Results: From Dec 12 to Dec 17 (2008), a total of 811 subjects were included, mean age was 58 (SD 11) years of which 405 (50\%) were women. The 4 most frequently identified stroke symptoms were sudden onset of: speech disturbances $\mathrm{n}=636(78,4 \%)$, facial palsy $\mathrm{n}=450(55,5 \%)$, hemiparesis $435(53,6 \%)$, and visual disturbances $n=429(52,9 \%)$. The 3 most frequently identified stroke risk factors were: hypertension $\mathrm{n}=586(72,3 \%)$, history of cerebrovascular disease $\mathrm{n}=401$ $(49,5 \%)$, increased cholesterol $n=269(33,2 \%) .10 \%$ correctly identified 4 major stroke symptoms whereas only 1 person ranked diabetes, hypertension, and tobacco smoking as the most important stroke risk factors.

Conclusions: In this population one-in-ten correctly identified all 4 pre-defined major stroke symptoms; diabetes, hypertension, and tobacco smoking was ranked simultaneously by only one person.

\section{Epidemiology of stroke}

GENDER DIFFERENCES IN MORTALITY AMONG STROKE PATIENTS WITH AND WITHOUT ATRIAL FIBRILLATION AND OTHER RISK FACTORS K.M. Henriksson ${ }^{1}$, B. Farahmand ${ }^{2}$, S. Johansson ${ }^{3}$, S. Åsberg ${ }^{4}$, A. Terént ${ }^{4}$, N. Edvardsson ${ }^{3}$

${ }^{1}$ Department of Laboratory Medicine, Lund University, Lund, Sweden; ${ }^{2}$ Institute of Environmental Medicine, Karolinska Institute, Stockholm, Sweden; ${ }^{3}$ Sahlgrenska Academy at Sahlgrenska University Hospital, Göteborg, Sweden; ${ }^{4}$ Department of Medical Sciences, Uppsala University Hospital, Uppsala, Sweden

Objective: To examine gender differences in mortality in stroke patients with/without atrial fibrillation (AF), and the impact of CHADS2 score.

Design: 105,074 patients ( $~ 50 \%$ women) were identified in the Swedish Stroke Registry during the years 2001-2005. Mortality and morbidity data were retrieved from the Inpatient and the Cause of Death registers. Risk factors included in the CHADS2 score (congestive heart failure (CHF), hypertension, age $\geq 75$ years, diabetes and a previous stroke) were assessed. Cox regression models were used to estimate relative risk and 95\% confidence intervals (RR, 95\% CI)

Results: AF was found in 14724 (28\%) men (mean age 78.0 years), and 17097 (33\%) women, (mean age 82.1 years). Among AF patients, $7922(54 \%)$ men and $10317(60 \%)$ women died, whereas in non-AF patients $12754(34 \%)$ men and $13287(38 \%)$ women died during the mean follow-up of 2 years time. RR of death varied by presence of component parts in the CHADS2 score in similar manner in both genders. In AF patients, women had higher RRs than men for CHADS2 scores 1 (2.59 vs. 2.40$), 2$ (3.27 vs. 2.88$) 3$ (4.00 vs. 3.87$), 4$ (4.37 vs. 4.00$), 5$ (5.24 vs. 4.87$)$, and 6 (5.82 vs. 6.16). This difference was more emphasized in patients without AF, although at a somewhat lower risk level: CHADS2 1 (2.43 vs. 1.85), 2 (2.94 vs. 2.45$), 3$ (3.78 vs. 3.12$), 4$ (4.19 vs 3.63$), 5$ (5.84 vs. 5.10$)$, and 6 (8.02 vs. 8.01). Further adjustment for age as a continuous variable markedly attenuated the found associations. Finally, as compared to women, the RR of death in men was about 1.05 and 1.10 with and without AF respectively and independent of other factors studied.

Conclusions: Men had slightly higher death risk after adjustment for age. The RR of death associated with each of the risk factors included in the CHADS2 score was equal between genders. Combined effects of the risk factors expressed by CHADS2 score observed increasing RR of death, and this increase was somewhat stronger among women 
WITHDRAWN
Predictors of mortality in Georgian population do not differ markedly from those reported in other population-based registries.

15 Epidemiology of stroke

FUNCTIONAL STATUS AFTER ISCHEMIC STROKE IN A LOW COMPLEXITY HOSPITAL IN A RURAL LOCATION IN CHILE

D.E. Galdames ${ }^{1}$, P.A. Mellado ${ }^{2}$, D.G. Galdames ${ }^{3}$

${ }^{1}$ Hospital San Luis, Buin, Chile; ${ }^{2}$ Hospital Clínico, Facultad de Medicina de la Pontificia Universidad Católica de Chile, Santiago, Chile; ${ }^{3}$ Hospital Sótero del Río, Santiago, Chile

Background: Buin is a rural town located $40 \mathrm{~km}$ from Santiago, capital city of Chile. It has a small hospital with basic beds. The objective is to ascertain the functional status in patients with ischemic stroke treated in a low complexity setting.

Methods: Patients $>15$ years of age with ischemic stroke that were hospitalized between the 1st January 2007 and the 31st December 2007 were included. Patients were first evaluated in the Emergency Room, if stroke was suspected the patient was referred to the base hospital located in Santiago. Here the subject was evaluated by a neurologist who after a CT scan would confirm the diagnosis. The patient was then sent back to Buin Hospital and admitted in the internal medicine ward. Risk factors and in-hospital morbidity and mortality were recorded. The TOAST classification was used. The modified Rankin scale (mRS) was applied at discharge and after 6 months of follow up. All assessments were done by a general practitioner (DG).

Results: 43 patients (18 women), 40 patients completed the follow up. The age average was 72 years (46 to 97 years). TOAST classification: $2.3 \%$ large-artery atherosclerosis, $23.2 \%$ cardioembolism, $21 \%$ small-vessel occlusion, $2.3 \%$ stroke of other determined etiology, and $51.2 \%$ stroke of undetermined etiology; $62.8 \%$ of the latter category has an incomplete evaluation. $55.8 \%$ had a transthoracic echocardiogram, $2.3 \%$ a transesophageal echocardiogram and $37.2 \%$ a carotic duplex. $\mathrm{mRS}$ at discharge: $0-2: 30.2 \%, 3-5: 62.9 \%, 6: 6.9 \%$. mRS after 6 months: 0-2: $67.5 \%, 3-5: 5 \%, 6: 27.5 \%$.

Conclusions: This study contributes in diminishing the lack of information about functional status after ischemic stroke in developing countries. Compared with other studies there is less disability after 6 months probably due to a selection bias (an unknown percentage stays at the base hospital after the specialist's evaluation). Access to tests is very limited compared with developed countries and urban hospitals in Chile.

14 Epidemiology of stroke

PREDICTORS OF MORTALITY IN PATIENTS WITH FIRST-EVER STROKE IN TBILISI, GEORGIA: A PROSPECTIVE POPULATION-BASED STUDY A. Tsiskaridze ${ }^{1}$, M. Djibuti ${ }^{2}$, T. Vashadze ${ }^{1}$, I. $_{\text {Burduladze }}^{3}$, I. Gauarashvili ${ }^{3}$, S. Apridonidze ${ }^{3}$, R. Shakarishvili ${ }^{1}$, P. Michel ${ }^{4}$

${ }^{1}$ Department of Neurology and Neurosurgery, Tbilisi State University, Tbilisi, Georgia; ${ }^{2}$ School of Public Health, Tbilisi State Medical University, Tbilisi, Georgia; ${ }^{3}$ Institute of Neurology, Tbilisi, Georgia; ${ }^{4}$ Department of Neurology, CHUV, Lausanne, Switzerland

Background: Stroke is one of the major public health problems worldwide However, information about epidemiological patterns of the disease in developing countries is scarce. The purpose of the present study was to determine predictors of mortality after stroke using the data from the joint Swiss-Georgian project on stroke epidemiology in Tbilisi, capital of Georgia, a country with transition economy in the South Caucasus region.

Methods: We followed-up a cohort of 233 first-ever stroke patients identified prospectively from 2000 to 2003 in Sanzona district of Tbilisi. All cases of mortality in the cohort were registered and analyzed by using overlapping sources of information and standard diagnostic criteria.

Results: Since November 2000 to date, 183 (79\%) stroke patients have died One-month case-fatality was $35 \%$. Fifty-one percent of patients died at one year. By using multivariate Cox proportional-hazards analysis, predictors of early $(\leq 1$ month from index stroke) mortality were hemorrhagic nature of stroke (Hazard Ratio HR 3.2; 95\% Confidence Interval CI 1.3-11.8) and severe initial neurological deficit as defined by NIHSS $>7$ (HR 2.6; 95\%CI 1.2-19.5), while predictors of intermediate ( $>1$ month and $\leq 1$ year) mortality were diabetes mellitus (HR 1.8; 95\% CI 1.2-26.0), cardiac comorbidity (HR 1.5; 95\%CI 1.1-21.9) and advanced age (>65 years) (HR 1.4; 95\%CI 1.1-24.3). Late (>1 year) mortality was associated merely with advanced age (HR $2.1 ; 95 \%$ CI $1.4-18.3$ ) and cardiac comorbidity (HR 2.5; 95\%CI 1.3-20.1)

Conclusion: Mortality after stroke in Tbilisi, Georgia is substantially high. This may be due to inadequacy of stroke service and shortages in the secondary preventive measures, which requires special attention of public health authorities.
16 Epidemiology of stroke

\section{IMPACT OF CAROTID PLAQUE ECHOLUCENCY ON CEREBRAL} EMBOLISM DURING CAROTID ARTERY STENTING M. Rosenkranz ${ }^{1}$, S. Havemeister ${ }^{1}$, O. Wittkugel ${ }^{2}$, G. Thomalla ${ }^{1}$, A. Krützelmann ${ }^{1}$, J. Fiehler ${ }^{1}$

${ }^{1}$ Dept. of Neurology, University Medical Center Hamburg-Eppendorf, Hamburg, Germany; ${ }^{2}$ Dept. of Neuroradiology, University Medical Center Hamburg-Eppendorf, Hamburg, Germany

Background: Carotid artery stenting (CAS) is associated with the risk of stroke. A better understanding of specific risk factors may help to reduce the overall risk of CAS. We addressed the role of carotid plaque echolucency as potential risk factor measure of echogenicity, the gray scale median (GSM), in 27 patients with symptomatic high-grade carotid stenosis that were scheduled to undergo CAS Dual-frequency transcranial Doppler ultrasound was used to detect solid cerebral microemboli during CAS.

Results: Solid cerebral microemboli were detected during 17 of 27 CAS procedures. The GSM of the target plaque was lower in patients with intraprocedural embolism (37.9 \pm 20.8$)$ than in those without $(58.2 \pm 25.7)(\mathrm{p}=0.040)$. A GSM of 50 gave the greatest separation between plaques with a higher and a lower probability of intraprocedural embolism: the proportion of patients with embolism was $85 \%$ in CAS of echolucent plaques $(\mathrm{GSM}<50)$ and $42 \%$ in CAS of echogenic plaques $(\mathrm{GSM} \geq 50)(\mathrm{p}=0.031)$

Conclusions: CAS of both echolucent and echogenic carotid plaques may be associated with cerebral embolism, particularly CAS of echolucent plaques. Plaque echolucency alone does not reliably identify patients at particularly high risk of intraprocedural embolism but should be considered as one of a broad panel of risk factors of CAS. for cerebral embolism during CAS.

Methods: We evaluated carotid plaque echolucency by use of a computer-assisted 
17 Epidemiology of stroke

PREVALENCE OF PERIPHERAL ARTERIOPATHY IN ACUTE STROKE PATIENTS - PARIS STUDY

H.B. Huttner ${ }^{1}$, M. Köhrmann ${ }^{1}$, C. Mauer ${ }^{1}$, H. Lücking ${ }^{2}$, S. Kloska ${ }^{2}$, P.D. Schellinger ${ }^{1}$

${ }^{1}$ Department of Neurology, University of Erlangen, Erlangen, Germany;

${ }^{2}$ Department of Neuroradiology, University of Erlangen, Erlangen, Germany

Background: There are only few data on the association of peripheral arteria disease (PAD) and stroke. Therefore we initiated the PARIS study (PARIS Peripheral Arteriopathy Rate In Stroke) to assess the prevalence of PAD in acute stroke patients.

Methods: This ongoing prospective, controlled, open, cohort study enrolls all consecutive adult patients with acute ischemic (including TIA) or hemorrhagic stroke as well as concurrent non-stroke and non-TIA patients. Upon admission to our stroke- or neurocritical care units assessment of the ankle brachial index (ABI) using the "boso-ABI-system 100" (www.boso.de) was performed. As an exclusion criteria congestive heart failure was defined.

Results: In the following we would like to present preliminary findings of the first 309 stroke patients (80 TIA, 187 ischemic, and 42 hemorrhagic strokes) and 50 controls who were matched for age. The median age was 67 (32-94) years and did not differ among the three groups. The prevalence of PAD in the control group was $16 \%$. Prevalence of PAD was higher in stroke patients (TIA: $18.75 \%$, ischemic stroke: $27.27 \%$, and hemorrhagic stroke: $21.43 \%$ ). The higher prevalence of PAD in ischemic stroke patients was significant $(\mathrm{p}<0.01)$ and further differentiation between lacunar and territorial infarct patterns revealed a higher prevalence of PAD in non-lacunar strokes $(\mathrm{p}<0.01)$.

Conclusion: To the best of our knowledge the PARIS study represents the first systematic investigation of the prevalence of PAD in stroke. By now, it appears that ischemic stroke patients suffer from PAD more frequently as compared to TIA patients and those with intracerebral hemorrhage, and PAD seems to be more common than in non-stroke patients. (ClinicalTrials.gov Identifier: PARIS Peripheral Arteriopathy Rate In Stroke: NCT00794066).

\section{Epidemiology of stroke}

\section{STROKE EPIDEMIOLOGY AND ONE-MONTH FATALITY IN AN IRANIAN CITY}

A. Delbari ${ }^{1}$, J. Lokk ${ }^{1}$, M. Rahgozar ${ }^{2}$, S.S. Tabatabaei ${ }^{2}$, R. Salman Roghani ${ }^{2}$ ${ }^{1}$ Karolinska Institute, Stockholm, Sweden; ${ }^{2}$ University of Social Welfare \& Rehabilitation, Tehran, Iran

Background: Stroke is one of the common causes of disability and death in Iran About $10 \%$ of all deaths in all age groups are due to stroke. This study evaluated stroke patients profile with respect to rate, risk factors and one-month fatality in an Iranian city.

Method: A retrospective, hospital based study on all stroke patients older than 45 years admitted to all general hospitals in the city of Qom throughout the year of 2001.

Results: 460 patients older than 45 years and 37 patients below 45 years were admitted and diagnosed as stroke during one year. Annual stroke rate was estimated to 338 per $100,000(95 \%$ CI, 300-360) inhabitants older than 45 years. The annual rate of stroke was 344 per 100,000 (95\% CI, 340-348) when adjusted to the Iranian population and 384 per 100,000 (95\% CI, 381-386) when adjusted to the European population. Stroke subtypes were ischemic infarction $75 \%$ (thrombotic $55.7 \%$, embolic $19.8 \%$ ), intracranial hemorrhage $20.7 \%$, subarachnoid hemorrhage $3 \%$, and undetermined $1.3 \%$. Main risk factors were hypertension in $74.6 \%$ and diabetes in $55.7 \%$. Mortality rate was $24.6 \%$ within the first month and the average length of stay at hospital was 7.8 days.

Conclusion: Stroke incidence, subgrouping of stroke types, and one-month mortality rate were comparable to most developing countries but stroke incidence was higher than in Western countries. Hypertension and diabetes mellitus were considerably more frequent in our studied stroke patients than in other developing countries. Our findings need to be addressed in future health education programs in Iran identifying patients at risk and focusing on more aggressive prevention programs to lower stroke incidence.
DOCTORS' CHOICE OF ANTITHROMBOTIC THERAPY AFTER ISCHEMIC STROKE; A COHORT STUDY OF 87111 PATIENTS REPORTED TO THE SWEDISH STROKE REGISTER (RIKS-STROKE)

S. Åsberg ${ }^{1}$, K.M. Henriksson ${ }^{2}$, B. Farahmand ${ }^{2}$

${ }^{1}$ Department of Medical Sciences, Uppsala University Hospital, Uppsala,

Sweden; ${ }^{2}$ Department of Laboratory Medicine, Lund University, Lund, Sweden

Background: In this study we investigate choice of antithrombotic (AT) therapy in relation to prior bleeding events (BE) among patients with ischemic stroke (IS), with the aim to provide guidance to a more adequate AT treatment in the future.

Methods: A cohort of 87111 IS patients was identified in the Swedish Stroke Register between 2001 and 2005. Data on BE during 1987-2005 were retrieved from the Swedish Hospital Discharge Register. Analyses were conducted for antiplatelet (AP) and anticoagulant (AC) therapy and adjusted for age, sex and AT on hospital admission. Odds ratios (OR) and 95\% Confidence Intervals (CI) were calculated using logistic regression models.

Results: Overall, the use of AP increase from $43 \%$ on admission to $76 \%$ at discharge, corresponding figures for $\mathrm{AC}$ being $6 \%$ and $11 \%$. Patients with $\mathrm{BE}$ requiring hospitalisation at any time prior to IS had a $22 \%$ (CI: 20-25\%) less likelihood of being discharged with AP therapy. The likelihood declined by $40 \%$ (CI: $33-46 \%$ ) if the bleeding was intracranial (IC) and $24 \%$ (CI: 20-28\%) if the bleeding was gastrointestinal (GI). The decline in likelihood was particularly strong the BE had occurred during the year preceding stroke; $47 \%$ (CI: $42-51 \%$ ) for al types of BEs, $77 \%$ (CI: $68-83 \%$ ) for IC events and $42 \%$ (CI: $34-49 \%$ ) for GI events. The likelihood of AC therapy was reduced by $11 \%$ (CI: $5-16 \%$ ) for any prior BE, by $47 \%$ (CI: $33-57 \%$ ) for IC events and by $18 \%$ (CI: $10-23 \%)$ for GI events. BEs during the most recent year did not significantly change the use of AC therapy.

Conclusion: Doctors" choice of antiplatelet therapy after ischemic stroke is significantly affected by the bleeding history of the patients, in particular by recent bleeding events. The lower impact of a history of non-IC bleedings and of recent events on anticoagulant therapy might be explained by ist higher potency and thus less frequent use.

\section{Epidemiology of stroke}

PRE-HOSPITAL DELAY FACTORS IN ISCHEMIC STROKE PATIENTS F. Moniche, M.D. Jiménez, A. Palomino, A. Cayuela, S. Vilaplana, J. De La Torre, E. Vigil

Hospital Universitario Virgen del Rocio, Seville, Spain

Background: Although since the publication of ECASS-III trial the therapeutic window for thrombolytics in ischemic stroke is wider, it is still important to focus our efforts on reducing time to treatment on these patients, as "time is brain" is still valid. Our aim is to analyze the reasons for arriving late to the Emergency Room (ER) in patients with an ischemic stroke.

Methods: During the year 2006, every stroke case in a tertiary hospital (target population, 1.000.000) with a pre-hospital Stroke Code System, was consecutively recorded. Wake-up strokes and strokes occurring on in-hospital patients were excluded. Patients were divided into two groups by symptoms-to-door time (SDT): within 4.5 hours and more than 4.5 hours. Baseline characteristics (age, sex, vascular risk factors), type of transport to hospital, stroke symptoms and the Glasgow Coma Scale (GCS) and Canadian Neurological Scale Score (EC) at the ER were analyzed.

Results: 498 ischemic stroke patients were registered. There were no differences on delay by sex, age or vascular risk factors. The fastest way to reduce delays was the use of Emergency Medical System (68.4\% within 4.5h SDT), vs going to hospital by their own vehicle $(42.1 \%)$, first consultation to a rural hospital $(42.9 \%)$ or to Primary Care $(37.6 \%), \mathrm{p}<0.0001$. Sensitive or motor symptoms were not associated with shorter SDT, whereas language disturbances $(57.9 \%$ within $4.5 \mathrm{~h}$ SDT vs $39.9 \%$ in patients without language symptoms, $\mathrm{p}<0.0001)$ and visual symptoms $(61.2 \%$ within $4.5 \mathrm{~h}$ SDT vs $47.2 \%$ without visual deficit, $\mathrm{p}=0.44)$ and more severe stroke by GCS $(\mathrm{p}=0.007)$ and $\mathrm{EC}(\mathrm{p}=0.001)$ were related to smaller delay.

Conclusion: Symptoms other than visual or language disturbances and the first consultation for assistant at Primary Care or at a rural hospital are the main reasons for a delay in symptoms-to-door time in stroke patients. Better knowledge of recognizing stroke symptoms and what to do in the general population is strongly needed. 
CARDIOVASCULAR VERSUS NON-CARDIOVASCULAR MORTALITY IN STROKE PATIENTS WITH AND WITHOUT ATRIAL FIBRILLATION AND THE IMPACT OF THE CHADS2 SCORE

K.M. Henriksson ${ }^{1}$, B. Farahmand ${ }^{2}$, S. Johansson ${ }^{3}$, S. Åsberg ${ }^{4}$, A. Terént ${ }^{5}$, N. Edvardsson ${ }^{6}$

${ }^{1}$ Department of Laboratory Medicine, Lund University, Lund, Sweden; ${ }^{2}$ Institute of Environmental Medicine, Karolinska Institute, Stcokholm, Sweden; ${ }^{3}$ Sahlgrenska Academy at Sahlgrenska University Hospital, Göteborg, Sweden; ${ }^{4}$ Department of Medical Sciences, Uppsala University Hospital, Göteborg, Sweden; ${ }^{5}$ Department of Medical Sciences, Uppsala University Hospital, Uppsala, Sweden; ${ }^{6}$ Sahlgrenska Academy at Sahlgrenska University Hospital, Göteborg, Sweden

Objective: To examine cardiovascular mortality in stroke patients with/without atrial fibrillation (AF), and the impact of CHADS2 score.

Design: In total 62145 patients were registered in the Swedish Stroke Registry during 2001-2003 and were followed through 2004 by the Inpatient and the Cause of Death registers. Risk factors included in the CHADS2 score (defined by assigning 1 point each for congestive heart failure (CHF), hypertension, age $>75$ years and diabetes plus assessing 2 points for a previous stroke) were assessed. Cox regression models were used to estimate relative risk and $95 \%$ confidence intervals (RR,95\% CI).

Results: During follow-up 22394 (36\%) died, of those 17251 (77\%) were due to CVD. The adjusted RR of CV mortality was 1.52 (1.48-1.57) and correspondingly 1.11 (1.05-1.18) for non-CVD mortality in patients with versus without AF. There was a trend towards an increased risk of death from CHADS2 score 0 to score 6. Age constituted the greatest RR for CV mortality, 3.08 (2.96-3.20). RR of CV mortality was 1.77 (1.70-1.83) for CHF, $1.32(1.28-1.36)$ for previous stroke and 1.07 (1.03-1.11) for diabetes and $0.91(0.88-0.93)$ for hypertension. Corresponding RR for non-CV mortality was 2.32 (2.18-2.48) for age, 1.66 (1.55-1.79) for CHF $1.64(1.55-1.75)$ for diabetes and $1.14(1.07-1.21)$ for a previous stroke and 0.79 $(0.75-0.84)$ for hypertension. Stratified analysis by AF revealed increased risk of death associated with almost all risk factors studied and was more pronounced among patients without $\mathrm{AF}$.

Conclusions: Three of four patients with a stroke died of CVD and more than hal of these had an AF-diagnosis. RR for patients with and without AF varied by cause of death and in general somewhat stronger associations were found in patients without AF. Diabetes had a greater impact on non-CVD mortality, which might be due to the lack of adequate treatment of the underlying diseases.

\section{Epidemiology of stroke}

\section{CLINICAL FEATURES OF ACUTE STROKE ASSOCIATED WITH LOW SOCIO- ECONOMIC STATUS}

G.K. Kerr ${ }^{1}$, P. Higgins ${ }^{1}$, K.R. Lees ${ }^{1}$, M.R. Walters ${ }^{1}$, S.K. Ghosh ${ }^{2}$, F. Wright ${ }^{3}$, P. Langhorne ${ }^{1}$, D.J. Stott ${ }^{1}$

${ }^{1}$ Cardiovascular and Medical Sciences, Faculty of Medicine, University of Glasgow, Glasgow, United Kingdom; ${ }^{2}$ Department of Medicine, Ayr Hospital, Ayr, Ayr, United Kingdom; ${ }^{3}$ Department of Medicine for the Elderly, Glasgow Royal Infirmary, Glasgow, Glasgow, United Kingdom

Background: Socio-economic (SE) deprivation is associated with increased risk of stroke and greater stroke severity. However the underlying causes for these associations are unclear. We aimed to determine the clinical factors associated with stroke in lower SE groups.

Methods: Prospective series of 467 consecutive patients with confirmed diagnosis of stroke or transient ischaemic attack (inpatients and outpatients), recorded at three Scottish Hospitals. Data collected; stroke severity (modified National Institutes of Health Stroke Scale, mNIHSS), medical history and vascular risk factors. SE status was derived from post-codes using Scottish Neighbourhood Statistics. Data were analysed in quartiles of socio-economic status.

Results: Stroke patients in the lowest SE quartile were younger (63.8 [SD 14.1] years) compared to those in the highest quartile $(72.3$ [12.7], $\mathrm{p}<0.0001)$ and had more severe stroke (mNIHSS 4 [IQR 2-6], versus 3 [1-5], $\mathrm{p}=0.036$ ). They were more likely to be smokers $(43 \%$ vs $23 \%, \mathrm{p}=0.002)$ and have a history of ischaemic vascular disease ( $56 \%$ vs $43 \%, \mathrm{p}=0.021$ ). There was no association of SE status with other measures of co-morbidity (Charlson index, number of medications), diabetes mellitus, total or HDL-cholesterol or blood pressure. The prior use of antiplatelets or statins in those with previous ischaemic vascular disease was similar in those from low compared to high SE groups; reduced rates of prior antihypertensive drug use were associated with low SE status, however this association became non-significant when adjusted for patient age in multivariate analysis.
Conclusions: Stroke amongst the most socio-economically disadvantaged occurs at a younger age, and tends to be more severe; it associates with greater co-morbidity in the form of prior vascular disease and with cigarette smoking. We found no evidence for low use of appropriate prior vascular prevention (antiplatelets, antihypertensives, statins) in association with low SE status.

\section{Epidemiology of stroke}

\section{AGE- AND GENDER-DIFFERENCE IN THE INCIDENCE OF ACUTE CHD} AND ISCHAEMIC STROKE IN FINNISH AND SWEDISH POPULATIONS M. Hyvärinen ${ }^{1}$, J. Tuomilehto ${ }^{2}$, S. Söderberg ${ }^{3}$, M. Eliasson ${ }^{3}$, C.D.A. Stehouwer ${ }^{4}$, Q. Qiao $^{2}$

${ }^{1}$ University of Helsinki, Helsinki, Finland; ${ }^{2}$ University of Helsinki, National Public Health Institute, Helsinki, Finland; ${ }^{3}$ Umeå University Hospital, Umeå, Sweden; ${ }^{4}$ Maastricht University Medical Centre, Maastricht, The Netherlands

Aims: We studied the age and gender difference between acute coronary heart disease (CHD) and ischemic stroke risk and examined to what extent such difference can be explained by known risk factors.

Methods: Data from Finnish and Swedish cohorts including 9278 individuals were collaboratively analyzed. Hazards ratios (HRs) (95\% confidence intervals) for CHD and stroke incidence were estimated using Cox-proportional hazards model.

Results: A total of $182(3.6 \%)$ women and 348 (8.4\%) men had first CHD and $129(2.5 \%)$ women and $137(3.3 \%)$ men ischemic stroke events. The multivariate adjusted HRs for incidence of CHD in men and women were 3.87 (2.49-6.02) and $1.71(1.07-2.74)$ at age 50-59 years, and $7.22(4.59-11.36)$ and $3.49(2.18-5.57)$ at age 60-69 years compared with women aged $40-49$ years. For ischemic stroke they were 2.64 (1.45-4.82) and $2.17(1.18-3.97)$ at age 50-59 years, and 5.19 (2.81-9.58) and 4.89 (2.67-8.97) at age 60-69 years, respectively.

Conclusions: The risk of acute CHD and ischemic stroke was higher in men than in women in all ages, but the gender difference was more marked for CHD than for ischemic stroke.

\section{Epidemiology of stroke}

LONG-TERM SURVIVAL AFTER STROKE - 30 YEARS FOLLOW UP G. Boysen ${ }^{1}$, J.L. Marott ${ }^{2}$, M. Grønbæk ${ }^{2}$, H. Hassanpour ${ }^{1}$, T. Truelsen ${ }^{1}$ ${ }^{1}$ Department of Neurology, Bispebjerg University Hospital, Copenhagen, Denmark; ${ }^{2}$ Copenhagen City Heart Study, Bispebjerg University Hospital, Copenhagen, Denmark

Background: Few have studied long-term survival after stroke. Knowledge about survival is important for estimating the current and future burden of stroke. The present study presents up to 30 years of follow-up of patients after a first-time-ever stroke.

Methods: Participants in the Copenhagen City Heart Study (CCHS) who experienced a first-time-ever stroke from 1978 to 2002 were continuously followed. Stroke events were validated using the World Health Organizations definition of stroke. Linkage to the Danish National Register of Death enabled identification of all patients who died before end of 2007. The register provided data on time of death and cause of death (COD). Survival in stroke patients was compared with the general population. Cox regression analyses adjusting for age and gender were used to compare survival in stroke patients in six 4 years' periods starting with 1978-1982.

Results: A total of 2,051 patients with first-ever stroke were registered, of whom $1,801(88 \%)$ died during follow-up. In the 65 to 72 years age group $46 \%$ survived 5 years after stroke. In the subjects $<65$ years $8 \%$ survived at 25 years. The relative risk of death after stroke decreased $44 \%$ (32\%-54\%) from 1978-1982 to 1998-2002 in analyses adjusted for age and gender in patients surviving the first week after symptoms onset. Life expectancy after stroke onset at age 70 was 4.8 years in men and 5.4 years in women in 1978-82; in 1998-02 it increased to 7.8 years in men and 8.8 years in women. In the general life expactancy at 70 years increased from 10.8 to 11.8 years in men and 14.0 to 14.6 years on women during the same period. COD were cerebrovascular disease in $37 \%$, other cardiovascular diseases $28 \%$, cancer in $12 \%$, and other causes in $23 \%$.

Conclusions: Long-term survival after stroke has gradually improved between 1978 and 2002. the gain in remaining life expectancy ater stroke exceeds that of the general population. 
THE INFLUENCE OF GENDER ON STROKE AND OUTCOME POST-STROKE J. Mc Manus, T. Adesina, M. Bhargava, B. Affley, S. Banerjee, R. Perry, D. Ames

St Mary's Hospital, London, United Kingdom

Introduction: While stroke incidence rates are lower in women across all age groups, it has been shown that women, who comprise an increasing percentage of our ageing population, have a worse outcome post-stroke than men. The aim of this study was to assess if there was any difference in outcome in women admitted to our hospital with acute stroke, compared with men.

Methods: Since August 2003, the clinical details of all stroke patients admitted to the Acute Stroke Unit at St. Mary's Hospital have been entered into a detailed database. This was interrogated looking at consecutive admissions from August 2003 to June 2008, to assess if there were any significant differences between men and women with regard to vascular risk factors, stroke type and and in particular clinical outcome.

Results: Of 1014 patients, 546 were male and 468 were female. The mean age (SD) of men was $68.64 \pm 13.71$ years and for women $73.92 \pm 12.33$ years $(\mathrm{p}<0.001)$ $75.1 \%$ of men had an admission Modified Rankin Score of 0, versus $68.5 \%$ of women $(\mathrm{p}=0.01)$. With regard to risk factors, men were more likely to have a history of smoking $(48.9 \%$ versus $23.1 \%, \mathrm{p}<0.001)$ and peripheral vascular disease $(8.9 \%$ versus $5.3 \%, \mathrm{p}=0.04)$, while women were more likely to have atrial fibrillation $(26.8 \%$ versus $15.8 \%, \mathrm{p}<0.001)$. There was no significant difference between men and women with regard to stroke type using the Bamford Classification. Outcome data was available for $91 \%$ of patients and showed no significant difference between men and women with regard to inpatient mortality, length of stay or likelihood of being discharged home.

Conclusion: The longer life-expectancy of women, and the direct relationship between advanced age and stroke risk suggests that older women will bear the major burden of the disease in the future. Female patients admitted to our hospital with an acute stroke are older and more dependent on admission than male patients. This does not, however, translate into female patients having a worse clinica outcome than men.

\section{Epidemiology of stroke}

\section{DO PATIENTS EXPERIENCE PROBLEMS AFTER BEING DISCHARGED FROM THE ER WITH A MINOR STROKE OR TIA? \\ M. Fens \\ Maastricht University, Maastricht, The Netherlands}

Background: Although it is known that patient who survive a stroke can experience problems in the long term, care in the chronic phase is not structurally organized We have initiated research into long term problems and possible health care models. Stroke patients receive no follow up care after being discharged to home from the ER, because it is believed that they experience no difficulties. The aim of this research project is to explore whether TIA or minor stroke patients experience problems after discharge and have a need for regular follow up care.

Methods: A new assessment tool was developed to measure stroke-related problems and need for care. The assessment tool was tested for feasibility and validity in a pilot study, which is part of a larger project. Patients were selected if they were diagnosed with a minor stroke or TIA, discharged home from the ER and were 50 years or older. The selected patients were interviewed by telephone.

Results: A total of 69 patients were selected six months after discharge, 63 patients of which were successfully interviewed. The data showed that 29 patients had five or more problems, mainly regarding cognition, communication, fatigue or medical complaints. Thirty-one patients responded to have cognitive deficits, especially with memory, and 28 patients had communication problems. Fatigue was present in 40 patients and pain complaints were present in 22 patients. Patients between 50 and 70 years old $(54 \%)$ had more cognitive deficits and more patients were fatigued than patients between 70 and 90 years of age $(46 \%)$. Women $(54 \%)$ experienced more psycho-emotional difficulties than men (26\%).

Conclusion: This research showed that about $50 \%$ of patients experienced problems regarding cognition, communication and fatigue, although they were discharged from the ER without follow-up care. Future research is necessary to identify prognostic indicators to identify stroke/TIA patients, who are at risk of experiencing long term problems.

27 Epidemiology of stroke

PHARMACOLOGICAL TREATMENT FOR SECONDARY PREVENTION IN A STROKE/TIA POPULATION

M. von Euler ${ }^{1}$, B. Nordin ${ }^{2}$, B. Wettermark ${ }^{3}$

${ }^{1}$ Department of Clinical Neuroscience, Karolinska Institutet, Stockholm, Sweden; ${ }^{2}$ Department of Pharmacological Biosciences, Division of

Pharmacokinetics and Drug Therapy, Uppsala University, Uppsala, Sweden; ${ }^{3}$ Department of Medicine, Center of Pharmacoepidemiology, Karolinska Institutet, Stockholm, Sweden

Introduction: Secondary prevention after stroke/TIA is known to be efficient but patient compliance to prescribed medications as well as physician's adherence to guidelines varies. We studied to what extent drugs recommended in guidelines for secondary prevention were dispensed to stroke patients in the whole region of Stockholm, Sweden (2 million inhabitants) before and 1-4 months after discharge. Method: We analysed data on medicines dispensed to all patients discharged after stroke/TIA during June 2006-July 2007. Patients $>18$ years with a primary diagnosis of ischaemic stroke (ICD codes I63.0-I63.9) or TIA (G45.9) were captured from the Swedish Hospital Discharge Register and linked to data on dispensed medicines from the National Prescribed Drug Register.

Result: A total of 5,687 patients were identified, $24 \%$ of these had a previous stroke/TIA. $14 \%$ of the patients died within 4 months of discharge. Antithrombotic agents were purchased by 82 and $43 \%$ of patients before and by 84 and $88 \%$ after discharge by patients with an earlier and a first-time stroke/TIA respectively. Hypertension was the most common diagnosed risk factor. Antihypertensives were purchased before admission by $82 \%$ of patients with recurrent and $68 \%$ of those with a first time stroke/TIA. After discharge, $75 \%$ and $71 \%$ of patients with recurrent and a first time stroke/TIA purchased antigypertensives respectively. Few patients $(23 \%)$ without a previous stroke/TIA used statins before admission while $46 \%$ purchased statins after discharge. For patients with a previous stroke/TIA 42 and $44 \%$ respectively purchased these drugs before and after discharge.

Conclusion: This study shows that it is feasible to perform large scale population based studies for whole regions on actually purchased medicines in patients with specific diseases. The compliance to the guidelines for secondary prevention of stroke/TIA was rather good, however, there is still a substantial room for improvement.

\section{Epidemiology of stroke}

\section{ROLE OF MICROEMBOLISM IN THE PATHOGENESIS OF WATERSHED INFARCTION}

R.R. Moustafa, I. Momjian-Mayor, P.S. Jones, S. Morbelli, D.J. Day,

F.I. Aigbirhio, T.D. Fryer, E.A. Warburton, J.C. Baron

University of Cambridge, Cambridge, United Kingdom

Background: Mainstream understanding of the pathogenesis of watershed infarction attributes it largely to hemodynamic impairment, particularly for deep watershed (DWS) infarction. Yet, a synergistic or even independent role for microembolism has been suggested. To test the role of microembolism in watershed infarction, we studied the relationship between, on one hand, the presence of microembolic signals (MES) on transcranial Doppler (TCD) and, on the other hand, the presence of DWS infarction and hemodynamic compromise, in patients with recently symptomatic severe carotid disease.

Methods: 16 patients (12 males, 4 females) with recent TIA/minor stroke and ipsilateral $>70 \%$ atherosclerotic carotid disease underwent brain MRI, oxygen-15 PET and TCD detection of MES using standard criteria. ROIs were defined for cortical and deep watershed areas. Symptomatic-to-contralateral hemisphere ratios of mean transit time (MTT), cerebral blood volume (CBV) and oxygen extraction fraction $(\mathrm{OEF})$ were compared in each ROI in patients with and without MES (MES+, $\mathrm{n}=8$; MES-, $\mathrm{n}=8$ ) using mixed-model ANOVA.

Results: 8/8 MES+ patients and 6/8 MES-patients had DWS infarcts. Across ROIs, both the MTT $(\mathrm{p}=0.003)$ and OEF $(\mathrm{p}=0.03)$ ratios showed significant elevation in MES- patients as compared to MES+ patients as well as to 10 healthy age-matched controls, with a strong trend to predominate in the centrum semi-ovale $(\mathrm{p}=0.06)$. In contrast, no significant difference was found between MES+ patients and controls. Conclusion: This study shows that in patients with severe carotid disease deep, watershed infarcts can develop even in the absence of hemodynamic compromise, strongly supporting the idea that DWS infarction can result from small emboli alone. Conversely, the presence of DWS infarcts and significant hemodynamic impairment in patients without MES confirms that in severe carotid disease DWS infarction can also occur independently of embolic phenomena. 
GLUTAMATE AND INFLAMMATORY CYTOKINES (IL-6, TNF-ALPHA) IN FOCAL CEREBRAL ISCHEMIA: A TRANSLATIONAL STUDY

P. Martinez-Sanchez, B. Fuentes

Stroke Unit. Department of Neurology. La Paz University Hospital. Universidad Autónoma de Madrid., Madrid, Spain

Background: Glutamate and cytokines are involved in the pathophysiology of cerebral ischemic damage. It is not clear if glutamate is a specific biomarker of brain damage or can be increased in other pathologies.

Aim: to evaluate the glutamate and cytokines (IL-6,TNFa) plasma levels in 3 groups: 1) Acute cerebral infarct (CI) humans, 2) rat model of focal cerebral ischemia, 3) rat model of tissular stress.

Methods: Groups: Humans (G1): Prospective case-control study. Cases (n=58): acute CI patients; Controls $(\mathrm{n}=19)$ : acute non-neurological diseases. Variables: glutamate, TNF receptor 1 (TNF-R1) and IL-6 within first $12 \mathrm{~h}$ after symptoms onset and at $72 \mathrm{~h}$; stroke severity (NIHSS), disease severity (APACHE); CI volume; 3-months functional status (mRS). Embolic stroke model (G2): Long-Evans (LE) rats. Subjects $(n=5)$ : internal carotid artery embolization with autologous clot. Tissular stress model (G3): LE rats ( $\mathrm{n}=5$ ) subjected to muscle compression in back paw during $180 \mathrm{~m}$. Variables (rats): glutamate, TNFa and IL-6 at 3 and $72 \mathrm{~h}(\mathrm{G} 2$ and G3); volume of ischemic lesion (H\&E) and neuronal death (TUNEL) (G2).

Results: Human: Glutamate and cytokines were increased over time. However, they did not correlate with infarction size and tended to be higher in non-neurological damage. A positive correlation was found between IL- 6 at $72 \mathrm{~h}$ and stroke severity as well as 3-months mRS $(\mathrm{P}<0.05)$ in cases; and between IL-6 and APACHE score on admission in controls $(\mathrm{P}=0.036)$. Glutamate levels did not correlate with disease severity. Rat: Glutamate and cytokines were increased over time, but did not correlate with infarct size and neuronal death. There were no differences between subjects and sham-operated rats. G3 showed higher glutamate levels at $3 \mathrm{~h}$ $(\mathrm{P}=0.08)$ and $72 \mathrm{~h}(\mathrm{P}=0.028)$ than $\mathrm{G} 2$ subjects.

Conclusion: Glutamate and cytokines are elevated in brain infarction. However they are not specific of cerebral ischemia since can be even more elevated in other tissues damage, both in human and animal models.

\section{Epidemiology of stroke}

\section{LONG TERM FOLLOW-UP OF 56 CONSECUTIVE PATIENTS WITH} CERVICAL ARTERY DISSECTION (CAD)

M. Kloss ${ }^{1}$, Ch. Lichy ${ }^{1}$, T. Brandt ${ }^{2}$, A. Grau ${ }^{3}$, C. Grond-Ginsbach ${ }^{1}$

${ }^{1}$ Department of Neurology, Heidelberg, Germany; ${ }^{2}$ Schmieder Kliniken,

Heidelberg, Germany; ${ }^{3}$ Department of Neurology, Ludwigshafen, Germany

Background: The long-term outcome of patients with a cervical artery dissection was usually reported to be favorable. However, prospective long-term follow-up studies of CAD patients of rigorous design were rare.

Methods: A consecutive series of 56 patients with cervical artery dissection (CAD) was prospectively included in the Neurology Department of the University of Heidelberg between June 1994 and December 1996. Clinical data upon the acute phase as well as risk factors and epidemiologic data were collected. Outcome after three months was assessed. Between 2006 and 2008 patients were invited to participate in a follow-up study.

Results: Long-term outcome was documented for 47 patients (84\% "responders"). Three patients had died, but the cause of their death was not related to CAD or stroke. 37 Patients came to the Neurology Department for an interview and a Doppler ultrasound examination, seven patients and relatives of the three deceased patients completed a questionnaire and were contacted by phone as well. Nine patients ("non-responders") could not be contacted, despite intensive and repeated inquiries.

The 3 month outcome of six out of the nine non-responders had been unfavorable (mRankin 2-5) whereas 38 of the responders had favorable outcomes (mRankin $0-1)$ after 3 months $(\mathrm{p}=0.02)$.

The 10 years outcome in this study was significantly associated $(\mathrm{p}=0.009)$ with the outcome after 3 month. Five patients suffered multiple dissections within the acute phase, four patients had a second CAD after a longer time ( $>1$ year).

Conclusion: Our study shows that recall bias is an important issue in long term studies of CAD. The findings suggest that patients with unfavorable 3 month outcomes are underrepresented in long term follow-up studies. The long term outcome of CAD might therefore be less good as indicated in most publications.
31 Epidemiology of stroke

\section{THE CORRELATION BETWEEN INFLAMMATORY MARKERS AND CAROTID ARTERY STENOSIS FEATURE OBTAINED BY DUPLEX SONOGRAPHY}

P. Puz, Z. Kazibutowska, E. Motta, A. Golba, A. Bal, M. Dębski

Medical University of Silesia, Katowice, Poland

Background: The main role of internal carotid artery stenosis in the pathogenetic mechanism of ischemic stroke is distal embolisation originating from unstable carotid plaque. Plaque morphology obtained by Carotid Duplex Sonography has been considered to define the plaque at risk for cerebrovascular events. The aim of this study is to find a correlation of several inflammatory markers with symptomatic carotid stenosis and signs of unstable carotid plaques in Carotid Color Coded Duplex Sonography.

Material and method: We investigated 65 patients (mean age: 66,29 $\pm 7,77 ; 45$ men) with internal carotid artery stenosis. 39 patients were symptomatic ( 26 stroke, 3 RIND, 8 TIA, 2 amaurosis fugax). With Carotid Duplex Sonography we evaluated the degree of stenosis (we divided it in three categories: $<60 \%, 60-80 \%,>80 \%$ ), surface and plaque morphology. Serum levels of WBC, monocyte count, ESR, Fibrinogen and CRP were measured.

Results: The mean values of all inflammatory markers were higher in symptomatic vs asymptomatic group (WBC: 7,70 vs $6,36 \mathrm{p}=0,001769$; monocyte count: 0,66 vs $0,52 \mathrm{p}=0,025037$; OB.: 14,33 vs $14,27, \mathrm{p}=0,962565$; fibrynogen: 3,57 vs $3,37 \mathrm{~g} / 1$, p=0,613218, CRP: 30,77\% elevated 19,33\% elevated, $p=0,22866)$. Patients with severe stenosis $(>80 \%)$ or ulcareted plaque surface had the highest values if inflammatory markers, but the results were statistically significant only for WBC. Patients with echolucent carotid plaques had higher mean values of inflammatory markers than patients with echogenic carotid plaques (WBC: 7,77 vs 6,54, $\mathrm{p}=0,010705$; monocyte count: 0,67 vs $0,53, \mathrm{p}=0,048132$; OB.: 16,45 vs $12,09, \mathrm{p}=0,018664$; Fibrynogen: 3,60 vs $3,38 \mathrm{~g} / \mathrm{l}, \mathrm{p}=0,206554)$

Conclusions: Inflammatory markers can be usefull in detection of patients with carotid plaques at high risk of destabilization. The degree of inflammatory process in carotid plaque can be measured with standard inflammatory markers. Echolucent carotid plaques are correlated with inflammation in the plaque.

\section{Epidemiology of stroke}

TRANSIENT NEUROLOGICAL ATTACKS: INCIDENCE AND VASCULAR RISK FACTORS IN NORTHERN PORTUGAL

M. Correia ${ }^{1}$, A. Tuna ${ }^{1}$, R. Magalhães ${ }^{2}$, M.C. Silva ${ }^{2}$

${ }^{1}$ Hospital de Santo António, Porto, Portugal; ${ }^{2}$ Instituto de Ciências Biomédicas de Abel Salazar (ICBAS), Universidade do Porto, Porto, Portugal

Background: Transient neurological attacks (TNA) are attacks of neurological symptoms/signs lasting less than 24 hours. They are known as TIA if focal, presumed to be of vascular origin and confined to a vascular territory. TNA not TIA can be focal, non-focal or mixed. TIA incidence is known, however TNA incidence and its clinical meaning is not completely understood. We determined the incidence of TNA in rural and urban population according to groups of symptoms/signs.

Methods: From October 1998 to September 2000 all patients with a first-everin-a-life-time TNA among a population of 104700 living in Northern Portugal were registered and observed by a neurologist soon after the event. Information on vascular risk factors (VRF) was collected. TNA were classified as TIA and TNA not TIA (Focal TNA when only focal symptoms were present and mixed TNA when both focal and non-focal symptoms occurred). Vertigo was registered when occurring alone or as predominant symptom and classified as Labyrinthitis, Benign Paroxysmal Positional Vertigo (BPPV) or Unspecified Vertigo (UV).

Results: Overall 547 patients were registered with mean age of 61 years $(s d=17)$ and $61 \%$ were women. Patients with Labyrinthitis were the youngest group (mean $=48.5, \mathrm{sd}=16$ ). High blood pressure was present in $50 \%$ of the patients, diabetes in 19\%, atrial fibrillation in 5\%, and high cholesterol in 38\% and smoking habits in 30\%. The prevalence of VRF in patients with UV was higher compared to the remaining groups. TNA crude incidence rate per 1000 persons year was 2.61 (95\% CI: 2.39-2.83) (TIA incidence 0.67) and 2.03 (95\% CI: 1.83-2.22) standardized to the European population. The incidence was slightly higher in urban compared to rural areas (2.64 vs. 2.49). Incidence rates ranged from 0.16 for UV to 0.69 for BPPV.

Conclusion: The prevalence of VRF in patients with TNA is high, particularly for those with UV. Incidence for TNA not TIA is tree times higher than for TIA. Supported by: FCT/FEDER project POCI/SAU-ESP/59885/2004 
EFFECT OF PRIOR TRANSIENT ISCHEMIC ATTACK (TIA) ON FUNCTIONAL OUTCOME AND SURVIVAL IN THE CASE OF SUBSEQUENT CEREBRAL INFARCTION. A POPULATION-BASED STUDY

Y. Béjot $^{1}$, C. Aboa-éboulé ${ }^{2}$, G.V. Osseby ${ }^{2}$, C. Binquet ${ }^{2}$, C. Bonithon-Kopp ${ }^{2}$, M. Giroud ${ }^{2}$

${ }^{1}$ Dijon Stroke Registry, E4 4184, University Hospital, Dijon, France; ${ }^{2}$ Dijon

Stroke Registry, EA 4184, University Hospital, Dijon, France

Background: We aimed to evaluate the potential neuroprotective effect of prior transient ischemic attack (TIA) on functional outcome and survival in the case of subsequent cerebral infarction.

Methods: We prospectively ascertained overall first-ever ischemic stroke occurring in Dijon, France (150,000 inhabitants) from 1985 to 2006. Cases were divided into lacunar and non-lacunar strokes. For each stroke patient, the history of prior TIA was recorded as was the duration ( $<$ or $>30$ minutes) and the interval between the TIA and the subsequent stroke ( $<$ or $>40$ weeks). Vascular risk factors were also collected. The functional outcome was evaluated at 1 month according to the Rankin score and survival was assessed at 1 month and 1 year. Comparisons were made between stroke patients with a history of TIA and those without.

Results: A total of 2692 ischemic stroke patients were recorded. Among these, $447(16 \%)$ had a history of TIA, 359 less than 4 weeks, and 88 more than 4 weeks before the stroke. Duration of TIA was $<30$ minutes for 275 patients and $>30$ minutes for the remaining 84 . The distribution of risk factors did not differ between stroke patients with or without previous TIA. Non-lacunar stroke patients with previous TIA had a more favourable functional outcome at 1 month ( $\mathrm{p}<$ $0.001)$ than did those without TIA, and better survival at 1 year $(\mathrm{p}<0.001)$ but not a 1 month, independently of the other risk factors. A short duration TIA $(<30$ minutes) and a short interval between the TIA and the stroke ( $<4$ weeks) were predictors of better functional outcome and survival.

Conclusion: Our large population-based study demonstrated that TIA prior to nonlacunar cerebral infarction resulted in a better functional outcome and improved 1 -year survival. These results confirm the potential ischemic tolerance phenomenon described in animal studies.

\section{Epidemiology of stroke}

STROKE OUTCOME IN GREEK PATIENTS AGED LESS THAN 45 YEARS K.N. Vemmos ${ }^{1}$, S. Vassilopoulou ${ }^{2}$, E. Manios ${ }^{1}$

${ }^{1}$ Acute Stroke Unit, Department of Clinical Therapeutics, University of Athens, Athens, Greece; ${ }^{2}$ Department of Neurology, University of Athens, Athens, Greece

Background: Limited data are available concerning the long-term prognosis in young adults after stroke. We aimed to determine the long-term functional outcome, the risk of stroke mortality and recurrence in a series of Greek young stroke patients.

Methods: Consecutive first-ever stroke patients, aged between 15 and 45 years and hospitalised in the stroke wards or referred to the stoke outpatient clinic of our tertiary care University Hospitals over a 10-year period, were prospectively included in a computerized observational databank. Demographic characteristics, stroke risk factors, baseline stroke severity (NIHSS), secondary prevention therapies were documented in all cases. Follow-up was routinely performed at the outpatient clinic of our institution. When patients failed to attend their regular (every 6-12 months) follow-up assessments at the hospital, they were contacted by phone call. The outcome events of interest during the follow-up period were recurrent stroke and death. Functional status was assessed by the modified Rankin Scale (mRS). Statistical analyses were performed using the Kaplan-Meier product-limit method and multivariate Cox regression analyses.

Results: We investigated a total of 280 cases fulfilling our study inclusion criteria (mean age $38 \pm 7$ years, male gender $57 \%$, median NIHSS-score 3.0 points $( \pm 6.17$ ) During a median follow-up period of 42 months (range 118 months) 22 patients died $(7.6 \%)$ and $27(9.6 \%)$ suffered from a recurrent cerebrovascular event. The cumulative 10-year mortality and stroke recurrence were $13.5 \%$ (95\% CI: $6.8 \%$ $20.2 \%$ ) and $21.1 \%$ (95\%CI: $11.3 \%-30.9 \%$ ) respectively. Functional independence (mRS $0-1)$ at the time of last follow-up was documented in $82.2 \%$ of 258 stroke survivors.

Discussion: Although the overall long-term prognosis appears to be favourable in young stroke patients and similar to population-based studies, the 10-year risks of recurrent stroke and death are not negligible.
35 Epidemiology of stroke

DIFFERENCE IN PROGNOSIS BETWEEN THE FIRST-EVER STROKE AND THE RECURRENT STROKE

K.B. Lee, I.M. Jang, J.S. Kim, H. Roh, M.Y. Ahn

Soonchunhyang University Hospital, Seoul, South Korea

Background: Few data is available regarding stroke recurrence in the patients with recurrent stroke. The purposes of this study were to compare the rates of stroke recurrence between the first-ever and the recurrent stroke.

Methods: From January 2005 to July 2008, all patients with a first-ever and recurrent stroke from SOONCHUNHYANG University Hospital were enrolled in a prospective and consecutive way. All patients were followed up prospectively at 3 months, 1 year, 2 years, and up to 3 years after stroke for mortality, and stroke recurrence.

Results: 505 patients $(74.3 \%$, mean age $66.8 \pm 12.7)$ had a first-ever stroke. The index stroke was recurrent in $175(25.7 \%$, mean age $68.7 \pm 11.6)$ of the 680 patients identified. The age, a history of hypertension and diabetes were significantly associated with recurrent stroke. The overall rates of non-fatal stroke recurrence were $2 \%$ at 3 months, $5 \%$ at 1 year, $8 \%$ at 2 years, and $17 \%$ at 3 years. The patients with recurrent stroke had significantly more events than those with first-ever stroke $(\mathrm{P}=.007)$. The prognostic factors for stroke recurrence were old ( $>65$ years) age, severe $(>50 \%)$ stenosis, and recurrent stroke per se for all stroke. Old ( $>65$ years) age, poor $(>3) \mathrm{mRS}$ at discharge, and severe $(>50 \%)$ stenosis were significant in the first-ever stroke group, whereas severe $(>17)$ NIHSS at admission, severe $(>50 \%)$ stenosis, and old (>65 years) age in the recurrent stroke group. Large artery atherosclerosis was more closely related with mortality $(32.3 \%$ versus $17.6 \%$, $\mathrm{P}=0.004)$ and stroke recurrence $(20.3 \%$ versus $6.8 \%, \mathrm{P}=0.001)$ in the patients with recurrent stroke than those with first-ever stroke.

Conclusion: Recurrent stroke is highly associated with old age, hypertension, diabetes, and large artery atherosclerosis subtype. The patients with recurrent stroke have more stroke recurrence in follow up period than those with first-ever stroke.

\section{Epidemiology of stroke}

\section{IMPROVING SUBTYPE CLASSIFICATION IN TOAST-DEFINED} CRYPTOGENIC STROKE IN THE NORTH DUBLIN POPULATION STROKE STUDY M. Marnane ${ }^{1}$, O. Sheehan ${ }^{1}$, C. Duggan ${ }^{1}$, A. Merwick ${ }^{1}$, N. Hannon ${ }^{1}$, E. Callaly ${ }^{1}$, D. Ni Chroinin ${ }^{1}$, G. Horgan ${ }^{1}$, J. Duggan ${ }^{2}$, L. Kyne ${ }^{2}$, J. Moroney ${ }^{3}$, A. Moore ${ }^{4}$, P.M.E. McCormack ${ }^{5}$, L. Daly ${ }^{6}$, P.J. Kelly ${ }^{1}$

${ }^{1}$ Neurovascular Clinical Science Unit, Mater University Hospital and University College Dublin, Dublin, Ireland; ${ }^{2}$ Department of Medicine for the Older Person, Mater University Hospital, Dublin, Ireland; ${ }^{3}$ Department of Neurology,

Beaumont Hospital, Dublin, Ireland; ${ }^{4}$ Department of Medicine for the Older Person, Beaumont Hospital, Dublin, Ireland; ${ }^{5}$ Department of Medicine for the Older Person, Connolly Hospital, Dublin, Ireland; ${ }^{6}$ School of Public Health and Population Science, University College Dublin, Dublin, Ireland

Introduction: Stroke recurrence rates and treatment strategies differ according to stroke mechanism and subtype. Refining subtype classification may thus improve the design of stroke clinical trials. Although the TOAST system is widely used, up to $40 \%$ of patients are designated as cryptogenic and inter-rater reliability is only moderate (kappa $\sim 0.5$ ). We investigated an alternative algorithm, the "Causative Classification System" (CCS) in a large population-based stroke cohort.

Methods: In a prospective cohort study in 294,592 North Dublin inhabitants, all first-ever ischaemic stroke (FES) patients were identified over one year and TOAST subtype classified after investigation completed. Using a standardised protocol, clinical and diagnostic data were abstracted from medical records and the web-based CCS algorithm applied.

Results: 381 FES cases were identified in the first year. Of these $112(29.4 \%)$ were "Stroke of undetermined aetiology" as per TOAST. This was due to 2 or more possible mechanisms in $31 / 381(8.1 \%)$ (Group A), incomplete evaluation in $36 / 381(9.5 \%$ ) (Group B) and negative evaluation in $45 / 381(11.8 \%$ ) (Group C). $80.6 \%(25 / 31)$ of group A, $13.9 \%(5 / 36)$ of group B, and $6.7 \%(3 / 45)$ of patients in group $\mathrm{C}$ had re-classification of subtype after application of the CSS algorithm (to Cardio-aortic [9/33, 27.3\%], Large Artery [8/33, 24.2\%], Small Artery [10/33, 30.3\%], Other Causes [6/33, 18.1\%]). Fewer patients were classified as undetermined aetiology using the CCS compared to TOAST (20.7 vs $29.4 \%$, $\mathrm{p}<0.001$ ). Inter-rater reliability was good (kappa $=0.7$ ).

Discussion: In this observational study, fewer strokes were classified as "Undetermined cause" using the CCS, particularly for patients with 2 or more potential aetiologies after investigation. In clinical trials, where mandatory investigations more frequently uncover competing aetiologies, the CCS may have additional utility, improving trial design and facilitating subtype analyses. 


\section{FIRST THREE YEARS MULTI-REGION STUDY ON STROKE EPIDEMIOLOGY IN OPEN POPULATION OF 25-74 YEAR-OLD MEN AND WOMEN IN RUSSIA \\ O.A. Klochihina, L.V. Stachovskaya, V.I. Skvorsova \\ Russian State Medical University, Moscow, Russian Federation}

Stroke incidence rate in Russia is one of the highest in the world, and mortality rate from stroke goes third after heart diseases and turmours of all locations. Within 2001 in Russia there were conducted the multi-region stroke epidemiology index studies in districts in different climate and geografic areas with a Register method which showed difference of incidence, attack, mortality and case fatality from stroke (in borders of Health National project). The higher stroke epidemiology index was registered in eastern regions of Russia as compared to the western ones. Standardized according to the European standard, stroke rate of incidence was 2,78 per 1000 population in Moscow and 4,71 per 1000 population in the East regions of Russia, the onset of the stroke was 0,79 too 1,75 per 1000 population as compared to Moscow and Sachalin. First open studies on stroke epidemiology in open population of 25-74 years-old men and women in Russia were started in January 2009 and will be conducted during three years in twelve administrative regions of a large country, Russia. The purpose of the work is to study the rate of incidence, attack, mortality and case fatality from stroke in different regions of the country - in order to organize the proper treatment and prevention of an acute stroke. The study will be executed with a Register method according to uniform criteria of Methodological Instruction of Russian National Association of Fight against Stroke.

Conclusions: Results of first three years multi-region stroke epidemiology index studies in Russia will be given, and they will show main stroke risk factors in incidence, attack and case fatality of the stroke in a multi-national country.

\section{Epidemiology of stroke}

\section{COMPARISON OF TRANSCRANIAL DOPPLER WITH MAGNETIC RESONANCE ANGIOGRAPHY IN ACUTE CEREBRAL ISCHEMIA B.B. Boddu}

Nizam's Institute of Medical Sciences, Hyderabad, India

Background and purpose: The objective of this study was to determine the accuracy of Transcranial Doppler (TCD) study in comparison to Magnetic resonance angiography (MRA) in detecting intracranial arterial stenosis in patients with acute cerebral ischemia and to establish diagnostic criteria based on velocities to detect various degrees of intracranial arterial stenosis.

Methods: Consecutive patients with symptoms of acute $(<24$ hours) cerebral ischemia were studied with both TCD and MRA. Bedside TCD was performed using a standardized, fast-track insonation protocol before or shortly ( 5 hours) after MRA. The peak flow velocities of intracranial vessels were recorded. Severity of Middle cerebral artery (MCA) stenosis on MRA was classified as normal-mild $(<50 \%$ lumen diameter reduction), moderate $(50 \%-70 \%)$, and severe $(>70 \%$ to $99 \%)$. Severity of other intracranial vessels was classified as normal $-<50 \%$ and $>50 \%$ stenosis on MRA.

Results: A total of 150 patients with acute cerebral ischemia underwent neurovascular evaluation with both TCD and MRA. Due to absent window in $14.67 \%$ patients the final evaluation was done in 128 patients. The optimal TCD cutoff velocity for detection of $>50 \%$ MCA stenosis was found at peak systolic velocity $>140 \mathrm{~cm} / \mathrm{s}$ and for $>70 \%$ MCA stenosis it was $>180 \mathrm{~cm} / \mathrm{s}$. The cutoff TCD velocity to identify $>50 \%$ stenosis of Anterior cerebral artery (ACA) and Internal carotid artery (ICA) was $>120 \mathrm{~cm} / \mathrm{s}$, while for Posterior cerebral artery (PCA), Vertebral artery (VA) and Basilar artery (BA) it was $>100 \mathrm{~cm} / \mathrm{s}$.

Conclusions: In patients with acute ischemic stroke the accuracy of TCD in detecting intracranial vascular stenosis is comparable to MRA. TCD enables grading of the severity of intra cranial vascular (MCA, ICA, ACA, PCA, VA, BA) stenosis according to the flow velocity.
DIFFERENCES IN DEMOGRAPHIC CHARACTERISTICS AND RISK FACTORS IN PATIENTS WITH SPONTANEOUS VERTEBRAL ARTERY DISSECTIONS WITH AND WITHOUT ISCHEMIC EVENTS

M. Arnold ${ }^{1}$, R. Kurmann ${ }^{1}$, A. Galimanis ${ }^{1}$, H. Sarikaya ${ }^{2}$, C. Stapf $^{3}$, A. Huber ${ }^{3}$ D. Georgiadis ${ }^{2}$, U. Fischer ${ }^{1}$, H.P. Mattle ${ }^{1}$, M.G. Bousser ${ }^{3}$, R.W. Baumgartner ${ }^{2}$ ${ }^{1}$ Department of Neurology, University Hospital Berne, Berne, Switzerland; ${ }^{2}$ Department of Neurology, University Hospital Zurich, Zurich, Switzerland; ${ }^{3}$ Assistance Publique, Hôpitaux de Paris, Department of Neurology, University Hospitals Lariboisière, Paris, France

Background: Spontaneous vertebral artery dissection (sVAD) mainly causes cerebral ischemia with or without associated local symptoms or signs (headache, neck pain, cervical radiculopathy) or local symptoms and signs only.

Patients and methods: We compared the presenting characteristics of patients with single sVAD and ischemic events and those with local symptoms and signs only. Results: We included 186 consecutive patients with single sVAD with either cerebral ischemia $(n=165)$ or local symptoms and signs only $(n=21) ;(116$ men, $62 \%$ ).

Patients with sVAD and ischemia were older (mean age 43.6 years +9.9 SD versus 38.6 years $+9.0 \mathrm{SD} ; \mathrm{p}=0.027)$ and were more frequently current smokers $(43 \%$ versus $14 \% ; \mathrm{p}=0.012)$. In patients with $\mathrm{SVAD}$ causing no ischemia female sex $(71 \%$ versus $37 \% ; \mathrm{p}=0.002)$ and migraine without aura $(61 \%$ versus $21 \% ; \mathrm{p}=0.025)$ were more frequent.

After multivariate analysis there were independent associations between higher age (0.027) as well as current smoking $(\mathrm{p}=0.012)$ and patients with sVAD causing ischemia, and between female sex $(\mathrm{p}=0.013)$ and sVAD causing no ischemia. Conclusions: These results indicate that patients who are older and current smokers are at increased risk to suffer cerebral ischemia, whereas women with sVAD may have less often cerebral ischemia compared to men.

\section{Epidemiology of stroke}

ANALYSIS OF 86 CASES OF PERINATAL ISCHAEMIC STROKE

H. Platokouki, H. Pergantou, A. Komitopoulou, P. Xafaki, S. Aronis Haemophilia/Haemostasis Unit, "Aghia Sophia” Children's Hospital, Athens, Greece

As perinatal is considered an ishaemic stroke (PIS) occurring from 28th gestational week to 7 th or 28 th postnatal day (if neonatal events are included). Presumed PIS (PPIS) is referred to children who have a delayed presentation of symptoms but are considered as having had acquired the infarct in utero or around the time of birth. The data from the files of 86 children with ischaemic stroke (IS) referred to our Unit for laboratory investigation of thrombophilia (FVLeiden, FII20210A, MTHFR C677T/A1298C, APCR, Proteins C/S, Antithrombin, FII, VII, VIII, XII, vWFAg, plasminogen, PAI, lupus anticoagulant) or therapeutic intervention, were analyzed. Twenty four cases were neonates (age $<28$ days)-Group I, and 62 had PPIS (age at diagnosis 5-96 months)-Group II. The proportion of males was significantly higher than that of females in both Groups (68.1 vs $31.9 \%$ and 65.9 vs $34 \%$, respectively). Most insults occurred in the left middle cerebral artery. Multiple infarcts were observed on MRI in Group II. Seizures and hemiplegia were frequent presenting symptoms in Group I and II, respectively. An underlying disease or a triggering event were found in $39 \%$ and $44.4 \%$ of cases in Group I and $10.5 \%$ and $10.3 \%$ in Group II (odds ratio in Group I vs II: 7.14 and 6.54, respectively). The odds ratio for idiopathic ischaemic stroke in Group II vs I cases was 10.3, whereas for the presence of thrombophilic factors was 2.99. In Group I, $8.3 \%$ of the neonates developed hemiplegia, $12.4 \%$ had seizures, one third were normal. On the contrary, in Group II, only $8.1 \%$ of children were normal, $6.1 \%$ and $8.1 \%$ presented with seizures and developmental disorders, and $59.7 \%$ remained hemiplegic.

Conclusion: The cause of IS in neonates seems to be multifactorial, whereas in PPIS cases thrombophilic factors could be implicated. Nevertheless, since hemiplegia is a common manifestation, imposing significant burden in the family and the society, a better understanding of PIS is imperative.

\section{WITHDRAWN}


STROKE - SEASONALITY AND WEEKLY VARIATION IN HUNGARY (2005-2007)

I. Kriszbacher $^{1}$, J. Betlehem ${ }^{1}$, I. Csoboth ${ }^{1}$, I. Vranics ${ }^{1}$, K. Gabara ${ }^{1}$, I. Boncz ${ }^{1}$, A. Olah ${ }^{1}$, E. Zsigmond ${ }^{2}$, J. Bodis ${ }^{1}$

${ }^{1}$ Faculty of Health Sciences, University of Pecs, Pecs, Hungary; ${ }^{2}$ Faculty of

Health Sciences, University of Pecs, Zalaegerszeg, Hungary

The aim of our study was to find out if a weekly or seasonal change can be seen in the incidence of stroke during a three-year study period in Hungary, and if the occurrence of stroke is influenced by age and sex.

Methods: We analyzed stroke patients $(\mathrm{N}=154.855)$ received at clinics and hospitals in Hungary (2005-2007). Data were collected from the data-base of the National Health Insurance Fund based on ICD 10. A retrospective data analysis was run using SPSS 14.

Results: In Hungary the onset of stroke shows a weekly and a seasonal variation. In the seasonal variation, the peek period of stroke was found during the months of Spring, with lowest number of events during the Summer. There was a significant difference between number of events for each season $(\mathrm{p}<0.01)$. The weekly peek period of cerebral circulatory disturbance was found on Monday, with a gradually decreasing tendency towards the end of the week. In age groups we have only found a significant difference with consideration to weekly variation: decrease in the number of events during the weekend is significantly greater in the age group below the age of $60(\mathrm{p}<0.01)$. Differences between the sexes was similarly only found in the weekly variation of number of stroke events, with a marked difference between the first five days of the week and the weekend $(\mathrm{p}<0.01)$.

Conclusion: the results of our study show that the incidence of a stroke shows characteristic variation with consideration to seasons and the days of the week.

\section{Epidemiology of stroke}

\section{CORONARY INTERVENTIONS AND STROKE}

S.K.R. Raghunathan, P. Gupta, B. Richard, P. Khanna

Nevill Hall Hospital, Abergavenny, United Kingdom

Introduction: Coronary angiography and angioplasty are established in investigation and treatment of cardiovascular abnormalities. The evidence regarding stroke following these cardiac interventions is lacking, but this serious complication needs to be highlighted.

Methods: Review of literature was undertaken on:

- Incidence,

- Risk factors and pathogenesis

- Treatments

for stroke following cardiac interventions.

Medline was searched using the terms "Coronary angiogram" or "Angioplasty, Transluminal, Percutaneous Coronary" and "Stroke" from 1979 until 2007.

Results: Incidence. Stroke following coronary intervention occurs in less than $1 \%$, the rate increasing with age. Silent radiologic ischemic changes are more common. Both ischemic and haemorrhagic strokes can occur, but typical water-shed strokes due to hypo perfusion are not typically reported. The vertebro-basilar territory may be involved in nearly half the ischemic strokes.

Risk factors and pathogenesis. Several Risk factors and pathogenesis mechanisms have been postulated including spread of atherosclerosis, neuro-toxicity with dye or contrast reactions, calcific embolus, thrombo-embolism and micro-embolism from manipulation of catheters. Strokes have also been related with the duration of the procedure, volume of contrast and the approach but none being consistently reported.

Treatment. Recent small trials and case reports have indicated that treatment is possible with thrombolysis via intravenous or intra-arterial route via the catheter.

Conclusion: Cardiac interventions are being used increasingly, with stroke being a rare but devastating complication. Patient characteristics and procedural risk factors like duration or route of procedure may contribute to strokes. Rapid treatment is possible with early identification resulting in good outcomes. A close liaison between cardiology and stroke teams is crucial for success.
44 Epidemiology of stroke

CONTRIBUTION OF DIFFUSION-WEIGHTED MAGNETIC RESONANCE IMAGING TO THE DIAGNOSIS IN PATIENTS WITH TRANSIENT ISCHEMIC ATTACK

F. Purroy $^{1}$, M.I. Gil ${ }^{2}$, R. Begué ${ }^{2}$, A. Quílez ${ }^{1}$, G. Piñol-Ripoll ${ }^{1}$, J. Díez ${ }^{3}$, C. Vicandi ${ }^{3}$, J.J. Sanahuja ${ }^{1}$, L. Brieva ${ }^{1}$

${ }^{1}$ Stroke Unit, Hospital Universitari Arnau de Vilanova, Universitat de Lleida, Lleida, Spain; ${ }^{2}$ Institut de Diagnòstic per la Imatge, Hospital Universitari Arnau de Vilanova, Lleida, Spain; ${ }^{3}$ Institut de Diagnòstic per la Imatge, Hospital Universitari, Arnau de Vilanova, Lleida, Spain

Objective: Diffusion-weighted magnetic resonance imaging (DWI) is a sensitive diagnostic tool for detecting acute ischemic lesions in patients with transient ischemic attacks (TIAs). The usefulness of the presence or absence of DWI abnormalities in the diagnostic workup of TIA patients still remains controversial. It would be interesting to know how the presence of DWI abnormalities influences the diagnosis of TIA patients

Methods: Two hundred consecutive TIA underwent diffusion-weighted brain imaging (DWI) (3.8 \pm 1.7 days after symptoms onset). The presence of acute ischemic lesions (DWI abnormalities) was related to clinical features and etiology.

Results: DWI abnormalities were identified in 94 (47\%) patients. Logistic regression model identified only motor impairment (OR 1.49 , 95\% CI 1.11 to 1.99, $\mathrm{p}=0.007$ ) as independent predictor of acute ischemic lesions. There were no differences among etiologic subtypes. Distribution of DWI lesions was: cortical 27 , subcortical 27, scattered lesion in one artery territory 31 and in multiple territories 9 . We observed lesion pattern non corresponding to clinical symptoms in $14 \%$ of carotid territory symptomatology and in $65 \%$ of patients with undetermined territory symptoms (absence of cortical symptoms and proportional motor impairment).

Conclusion: Our data added evidence of the clinical utility of DWI in TIA patients. DWI changed the suspected vascular TIA localization and the suspected etiology mechanism in two of five cases with acute infarction.

\section{Epidemiology of stroke}

\section{RE-AUDIT TIMING OF SCAN IN STROKE PATIENTS}

S. Elghenzai

Ysbyty Gwynedd Hospital, Bangor, United Kingdom

This audit was carried out at Ysbyty Gwynedd Hospital Bangor that is situated in North Wales. this hospital is 450 beded and covers a large geogriphical area from Holyhead in the north to Tywyn in the south and from Pwllheli in the west to Llandudno in the east.There is a stroke ward in the hospital called prysor ward which is 15 beded ward. the stroke patients are first admitted in AMU (tryfan) ward from where they are transferred to prysor ward.

according to Royal College of Physician Guidlines 'brain imaging should be under taken as soon as possible in all patients, within 24 hours at most of onset of symptoms'. NICE Guidlines 68-Stroke also reiterates this standard. The first audit was done in 2006 to find out the time of CT Scan in patients with stroke. The results showed that out of 58 patients only $14 \%$ had thier CT Scan in 24 hrs while $86 \%$ had after 24 hrs which is well below the standard. After this results some changes were introduced in the system to speed up the process of CT Scan i.e.

1 - Signing the CT Scan form immediately when the patient is admitted in AMU.

2 - Sending form immediately to radiology departement.

3 - Introduction of blue stickers on CT Scan request form so that radiology departement are aware that these patients are to be CT scanned within $24 \mathrm{hrs}$

To close the loop we did a re-audit in june 2008 to find out whether we have achieved the standards or not. In this audit 55 patients were included out of which $37 \%$ of patients had thier CT Scan done within $24 \mathrm{hrs}$ of admission while $63 \%$ didnot have CT Scan within 24 hrs of admission.

This re-audit showed that there has been significant improvement as in the first audit only $14 \%$ of the patients had CT Scan done within 24 hrs of admission while in $200837 \%$ of patients had CT Scan within $24 \mathrm{hrs}$ of admission. Still there is a long way to go and more measures needs to be taken to achieve the standards which is that $100 \%$ of the patients should have thier CT Scan in $24 \mathrm{hrs}$ of admission. 
PRETREATMENT WITH ANGIOTENSIN-CONVERTING ENZIME INHIBITORS (ACE) OR ANGIOTENSIN-RECEPTOR BLOCKERS (AT-1) IMPROVES OUTCOME AFTER ISCHEMIC STROKE

L.C. Álvaro ${ }^{1}$, J.J. Timiraos ${ }^{2}$, I. Escalza ${ }^{3}$, J.C. Martín ${ }^{4}$, F. Sádaba ${ }^{1}$

${ }^{1}$ Basurto Hospital, Bilbao, Spain; ${ }^{2}$ Txagorritxu Hospital, Vitoria, Spain;

${ }^{3}$ Galdácano Hospital, Galdácano (Vizacaya), Spain; ${ }^{4}$ Clínica Santa María de la Asunción, Tolosa (Guipúzcoa), Spain

\section{Background: AND}

Objectives: Angiotensin-converting enzyme (ACE) inhibitors and angiotensinreceptor (AT-1) blockers reduce recurrent ischemic strokes by mechanisms not linked to reducing blood pressure (BP). In addition, neuroprotection has been proven in pretreated animal models of brain ischemia. On these grounds, we tested the hypothesis that patients taking these drugs before their stroke onset could have less severe ischemic strokes and a better outcome.

Methods: We prospectively included consecutive patients with a well defined ischemic stroke (first-ever) of $<24$-hour duration, admitted to four general hospitals in a mid-sized area. NIHSS score at presentation was used as a measure of stroke severity. We also recorded demographic data, vascular risk factors, BP, Oxfordshire classification, mechanisms (TOAST criteria) and medications. At $72 \mathrm{~h}$ or before discharge NIHSS score was again recorded to define progression ( $>3$ points higher) and functional status (NIHSS $>14$ ). Outcome at 90 days is favourable if Barthel Index $(\mathrm{BI})>95$ and modified Rankin scale $(\mathrm{mRS})=0-1$ (independent). Data were analyzed using univariate methods and a logistic regression with the dependent variable of favourable outcome (FO).

Results: We included 235 patients (mean age 74.3 years, 121 men). 81 (34.5\%) were pretreated with ACE/AT-1 (G1; GO: untreated). NIHSS score was not lower on admission in G1 (NIH>14: G0 14.3\%, G1 13.6\%); the rate of progression was neither reduced. Significant difference on BP (admission, discharge) was not proved. FO was more common in pretreated cases (G1: 67.5\%, GO: 52.35 , $\mathrm{p}=0.028)$. Predictors of FO were age $(\mathrm{p}=0,002)$ and pre-treatment with ACE/AT- 1 $(\mathrm{p}=0,003)$.

Conclusion: Patients on ACE/AT-1 before an ischemic stroke have a favourable outcome compared with untreated cases. This prognosis is not influenced by stroke severity, or by differences in BP. Results are robust, although their validity should be confirmed with randomized controlled trials.

\section{Epidemiology of stroke}

STROKE LESION PATTERNS REFLECT DIFFERENCES IN RISK FACTOR PROFILES BETWEEN WOMEN AND MEN

A. Förster, A. Gass, R. Kern, M. Wolf, C. Ottomeyer, K. Zohsel,

M.G. Hennerici, K. Szabo

Universitätsklinikum Mannheim, University of Heidelberg, Mannheim, Germany

Background: Differences between women and men in relation to stroke are increasingly being recognized.

Methods: From July 2004 until June 2007237 acute ischemic stroke (AIS) patients were treated with rtPA within three hours after onset of symptoms in our stroke unit. Baseline characteristics, etiology, MRI stroke patterns and clinical outcome of women were compared to those of men.

Results: Of 237 AIS patients (mean age 70.7 years), 111 (46.8\%) were women and $126(53.2 \%)$ were men. Women were older $(\mathrm{p}=0.001)$, but history of hyperlipidaemia $(\mathrm{p}=0.02)$, smoking $(\mathrm{p}=0.02)$ and coronary heart disease $(\mathrm{p}<0.001)$ was less frequent than in men. Internal carotid artery (ICA) disease occurred more often in men $(\mathrm{p}=0.05)$, whereas atrial fibrillation $(\mathrm{AF})$ was observed more often in women $(\mathrm{p}=0.006)$. In men borderzone/small embolic and lacunar stroke was found

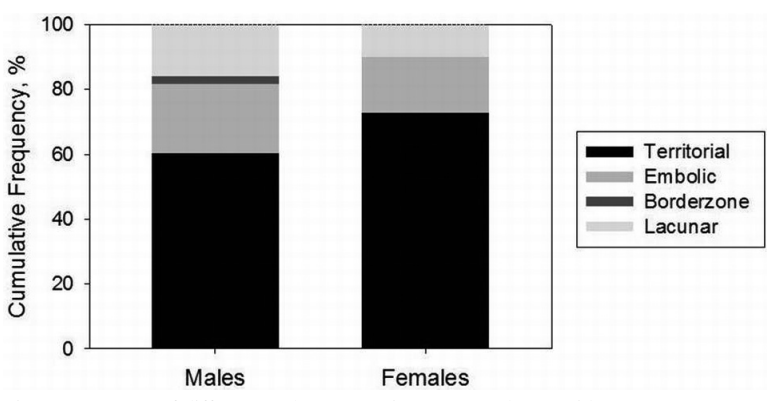

Fig. 1. Frequency of different stroke patterns in women and men with AIS. more frequently (39.7 vs. $27.2 \%$ ), while women showed a higher frequency of large territorial stroke (72.8 vs. $60.3 \%$ ). Baseline NIHSS (12.5 vs 11.3$)$, NIHSS at discharge (11.0 vs. 9.5), 3-month-outcome mRS, thrombolysis related (47.7\% vs. $40.7 \%)$ or independent complications $(32.4 \%$ vs. $30.2 \%)$ as well as mortality after three months $(13.5 \%$ vs. $9.5 \%)$ were similar in both groups.

Conclusion: The frequency of different stroke lesion patterns between sexes reflected the differences in etiology/risk factor profiles (women - cardioembolism; men - large and small vessel disease). Baseline characteristics, rates of rtPA related and independent complications as well as clinical outcomes were not different between women and men with AIS

\section{Epidemiology of stroke}

\section{ACUTE SPINAL CORD ISCHEMIA: MR FINDINGS}

C.L. Alblas, W. Bouvy

Medical Center Haaglanden, the Hague, The Netherlands

Background: Diagnosing spinal cord ischemia is based on clinical symptoms and MR imaging showing focal cord enlargement and "pencil like" hyperintensities on T2 weighted images. However, MR imaging is usually normal in the first hours after onset of symptoms. We will present our clinical and MR imaging findings in diagnosing spinal cord ischemia.

Methods: Eight consecutive patients with acute spinal cord ischemia were admitted in a two year period at the Department of neurology of the Medical Center Haaglanden in the Hague. Spinal MR imaging (1.5T) was performed, at least once, in all patients. Clinical data were retrieved from the patient's charts.

Results: Five women and three men with a median age of 51 years were admitted. Six patients had anterior spinal artery infarction and two patients transverse infarction. Causes were aorta descendens occlusion, vertebral artery dissection, hypotension during surgery, atherosclerosis and trauma. Five patients had MR imaging within 24 hours, which was normal in four patients. Repeated MR imaging showed in all patients focal cord enlargement and hyperintensities on T2 weighted images. Several days after onset of symptoms enhancement after gadolinium administration was seen in all patients. In three patients also diffusion weighted images were performed showing diffusion-restriction. In one patient diffusion restriction was seen on MR imaging 8 hours after onset, with normal T1 and T2 weighted images. Vertebral body infarction was seen in one patient

Conclusion: Spinal cord ischemia has a typical course on MR imaging over time. In the acute phase MR imaging is normal and after a few days spinal cord enlargement, hyperintensities on T2 weighted images and enhancement after gadolinium administration is seen. Repeated MR imaging is necessary in patients suspected of spinal cord ischemia with an early normal MRI. Diagnosing spinal cord ischemia remains a challenging task for neurologists and neuroradiologists.

\section{9}

Epidemiology of stroke

\section{MARITAL STATUS INFLUENCES PREVALENCE AND SEVERITY OF} STROKE IN MID-AGED PATIENTS

I.S. Joo, J.M. Hong, J.H. Lee, W.Y. Li

Department of Neurology, Ajou University Hospital School of Medicine, Suwon, South Korea

Background and purpose: Mid-aged stroke (40's and 50's) is an important social issue because the sufferers are in general actively working members of the society and family. Alterations of marital status have been associated with higher incidence and poorer prognosis of coronary heart disease in the mid-aged population. Up to now, however, the impact of marital status influencing the stroke occurrence has not been elucidated in mid-aged patients with ischemic stroke. We intended to examine the relationship between the marital status and characteristics of ischemic stroke patients.

Methods: Of all acute ischemic stroke patients who were less than 60 years old and consecutively admitted to a referral hospital between January 2006 and July 2008, a total of 249 eligible patients were enrolled excluding those younger than 40 . The subjects were divided into stable (married) and unstable marital status (divorce, dissolution and widowed) groups. Effects of the demographics, conventional stroke risk factors (hypertension, diabetes, smoking, previous stroke history or cardiac disease), and stroke mechanisms were separately examined for the age groups of $40 \mathrm{~s}$ and $50 \mathrm{~s}$.

Results: Of 249 eligible patients (mean age: 50.9 \pm 5.9 , male: $73 \%$ ), 106 were 40-49 years of age and 143 were 50-59 years of age. There were no significant differences in the baseline demographics, stroke risk factors, or stroke mechanisms. However, in the 40 to 49 age group, current smoking ( $72 \%$ vs. $48 \%, p=0.037$ ) and cardiac disease $(24 \%$ vs. $8 \%, \mathrm{p}=0.024)$ were significantly higher in the unstable compared to the stable marital. Also, initial stroke severity $(\mathrm{p}=0.016)$, 
cardioembolism proportion $(\mathrm{p}=0.011)$, and the proportion of $40 \mathrm{~s}$ male $(\mathrm{p}=0.003)$ were significantly higher in the unstable marital status group.

Conclusion: These findings suggest that unstable marital status might be an important trigger for the acute ischemic stroke occurrence, particularly for males in their 40s. As compared to the stable marital status group, initial stroke severity and cardioembolic mechanism were significantly higher in the unstable group, which was more distinctive in the males in their $40 \mathrm{~s}$.

50 Epidemiology of stroke

WITHDRAWN socioeconomic status may be one of the reason of stroke in low level culture and workless woman.

Key words: cerebrovascular accident; risk factors; retrospective research; gender

\section{2}

Epidemiology of stroke

PATENT FORAMEN OVALE, ATRIAL SEPTAL ANEURYSM AND STROKE: PATIENTS REVIEW

J.D. Pinho, M. Rodrigues, J.J. Cerqueira, R. Maré, C. Ferreira, J.R. Fontes

São Marcos Hospital, Neurology Department, Braga, Portugal

Introduction: Patent foramen ovale (PFO) and atrial septal aneurysm (ASA) have been described as stroke risk factors, especially for cryptogenic stroke. Causal relation is in discussion and the best treatment remains unsettled. Our goals were to characterize a population of stroke or transient ischemic accident (TIA) patients whose investigation revealed PFO/ASA and to correlate clinical aspects and treatment decisions with stroke recurrence, complications and functional outcome Methods: All patients with stroke or TIA of any cause and PFO and/or ASA (transesophageal or transtoracic echocardiography diagnosis), who came to our attention between August 2004 and November 2008, either in-hospital or consultation setting were included. Revision of clinical records and telephonic contacts were undertaken to collect clinical information.

Results: We included 45 patients, mean age 48 years; ASA patients were significantly older than isolated PFO patients $(\mathrm{p}=0.01)$. ASA was more frequent in women $(\mathrm{p}=0.01)$. In $24 \%$ the index event was a TIA. In $48,9 \%$ presentation was of a partial anterior circulation syndrome, and the most frequently involved arterial territory was cortical anterior $(26.7 \%)$. Stroke was classified as cryptogenic in $80 \%$ of patients. Anticoagulation was the treatment in $24,4 \%$ at presentation; 3 were submitted to PFO closure. Mean follow up time was 19,2 months (1-60), 11\% of patients with cryptogenic stroke had recurrent ischemic events and 1 had a major hemorrhagic complication. No differences of recurrence were found considering treatment, cardiac condition or etiological classification. At the end of follow up $93,3 \%$ had a modified Rankin scale score $\leq 2$.

Conclusions: Our young adult population reflects extensive diagnostic workup in this age group, but we cannot exclude the contribution of PFO/ASA as a stroke risk factor, especially in younger ages. Clinical and imaging findings suggest the predominance of embolic stroke mechanism. Funcional outcome is favourable.

\section{Epidemiology of stroke}

\section{FREQUENCY OF INTRACRANIAL VESSEL ANOMALIES IN} CEREBROVASCULAR PATIENTS

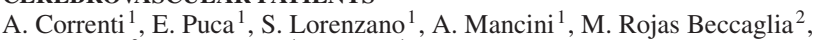
G.F. Gualdi ${ }^{2}$, M. Prencipe ${ }^{1}$, D. Toni ${ }^{1}$

${ }^{1}$ Unità di Trattamento Neurovascolare, Department of Neurological Sciences, University of Rome "La Sapienza", Rome, Italy; ${ }^{2}$ Radiology, Policlinico Umberto I, University of Rome "La Sapienza", Rome, Italy

Background: The impact of intracranial cerebral artery anomalies is not enough known. The aim of this study is to describe the frequency of intracranial arterial known. The

Methods: We analyzed retrospectlively a continuous series of 502 patients admitted to the stroke unit of our teaching hospital for whom a cerebral MRI and MR-Angiography were performed.

Results: Overall, 291 (58\%) patients were male, with a mean ( \pm S.D.) age of $65 \mathrm{aa}( \pm 15)$. A diagnosis of ischemic stroke was done in $57 \%$ of cases, of TIA in $16 \%$ of patients, hemorrhagic stroke in $10.8 \%$, subarachnoid hemorrhage in $1.1 \%$, cerebral venous sinus thrombosis in $1 \%$ and $14 \%$ of patients had other CNS diseases. Intracranial cerebral artery anomalies were found in 86 patients $(27.8 \%)$. The posterior cerebral artery presented $38(44 \%)$ anomalies: fetal origin from homolateral carotid cerebral artery in 32 cases (15 on the right side, 12 on the left side, 5 on both side) and hypoplasia in 6 ( 3 on the right side, 2 on the left side, 1 on both side). The anterior cerebral artery presented hypoplasia in 15 cases $(17,4 \%): 12$ on the right side, 3 on the left side. The posterior communicants artery was hypoplastic in 13 patients $(15 \%): 3$ on the right, 2 on the left, 8 on both side. The vertebral artery was hypoplastic in 7 cases $(8,1 \%): 1$ on right side and 6 on left side. Among $13(7,4 \%)$ remaining anomalies, we found 1 hypoplastic anterior communicants cerebral artery, and 1 case of Moya-Moya like vessel anomalies.

Discussion: In our casistic the fetal origin of posterior cerebral artery is the most frequent Circle of Willis variant. Interestingly, when it was present on the right side it is always associated with a hypoplasia of another right artery, on the contrary when it is on the left side it was not associated with any other vessel anomalies Further studies are necessary to better understand the influence of these vesse anomalies on the site of acute vascular lesion and on outcome of stroke patients. 
VASCULAR RISK FACTORS DIAGNOSED AFTER A TRANSIENT ISCHEMIC ATTACK AND THEIR INFLUENCE IN THE PRESENCE OF ARTERIAL DISEASE

P. Martinez-Sanchez, I. Sanz-Gallego, J. Fernández-Dominguez, L. Idrovo, M.J. Abenza

Stroke Unit. Department of Neurology. La Paz University Hospital. Autonoma de Madrid University. Madrid. Spain., Madrid, Spain

Background: Our aim is to study the vascular risk factors profile diagnosed after a transient ischemic attack (TIA), during the hospitalization in a Stroke Unit, as well as to determine its influence in the presence of intra/extracranial arterial stenosis. Methods: Patients with first-ever TIA were consecutively identified over a 2-year period (2006-2007). Variables analyzed: demographic data, previous vascular risk factors; diagnostic test results (brain TC/MRI, transcranial Doppler ultrasound, carotid duplex, blood analyses), vascular risk factors identified immediately after the TIA and confirmed at 3-months: High Blood pressure (HBP), atrial fibrillation (AF), diabetes mellitus (DM), dyslipidemia (DL) and silent myocardial infarction (SMI).

Results: A total of 161 patients were included, 90 male. Mean age $66.8 \pm 13.7$ years Frequency of previous vascular risk factors: 54\% HBP;16.8\% DM; 6.2\% AF; $4.3 \%$ valvulopathy, $38.5 \%$ DL, $5 \%$ peripheral arterial disease, $27.3 \%$ smoking, $12.4 \%$ coronary arterial disease or previous myocardial infarction. The $79.5 \%$ of patients had at least one previous vascular risk factor. Frequency of risk factors identified after TIA: $8.1 \%$ HBP; $1.2 \% \mathrm{DM}, 18 \% \mathrm{AF}, 12.4 \% \mathrm{DL}$; and $2.5 \%$ SMI. At least one vascular risk factor was diagnosed in $35.4 \%$ of patients. There were not differences between women and men. The $16.1 \%$ of patients presented significant intra/extracranial arterial stenosis. The de novo diagnosis of DM was associated with the presence of arterial stenosis $(\mathrm{P}=0,025)$. The frequency of risk factors identified after the TIA was similar among TIA and acute cerebral infarction patients treated during the same period.

Conclusions: The frequency of vascular risk factor diagnosed after a TIA is high. The diagnosis of de novo DM is associated to the presence of intra/extracranial arterial stenosis.

\section{Epidemiology of stroke}

\section{LONG-TERM CLINICAL OUTCOME AFTER APPROPRIATE TREATMENT BASED ON EMRGENCY MRI AND INTENSIVE REHABILITATION OVER THE 3 MONTHS FOR SYMPTOMATIC HYPERACUTE ISCHEMIC STROKE M. Nakazaki, T. Mori}

Shonan Kamakura General Hospital, Kamakura, Japan

Background: We aimed to investigate effectiveness of appropriate treatment ac cording to probable stroke subtypes on the basis of emergency MRI and intensive rehabilitation over 3 months for symptomatic hyperacute ischemic stroke and to find the most important predictor of long-term clinical outcome.

Methods: From Jan 1, 2006, to Feb 1, 2008, inclusion criteria for our retrospective analysis were neurological symptoms (National Institutes of Health Stroke Scale [NIHSS] score of 1 or more) on admission, emergency MRI for accurate diagnosis for ischaemic and vascular lesions, appropriate treatment started within $3 \mathrm{~h}$ of stroke onset, and intensive rehabilitation over 3 months. Patients' age, sex, treatment modality, NIHSS score on admission, NIHSS score at 7 days, and modified Rankin score (mRS) at 3 months were recorded. These factors were analysed to find the most important predictor of 3-month $\mathrm{mRS}$.

Results: Of 671 patients with ischemic stroke admitted to our hospital within $72 \mathrm{~h}$ of stroke onset, 140 were analyzed. NIHSS score on admission was 1-34. Immediately after emergency MRI, all patients were treated appropriately. 30 patients $(21.4 \%)$ had emergency recanalization (alteplase [rt-PA] or endovascular) for large-vessel occlusion. Median NIHSS score was improved from 8 on admission to 5 at 7 days $(\mathrm{p}<0.001)$. After intensive rehabilitation, 61 patients $(44 \%)$ had no disability at 3 months ( 0 or 1 of mRS) and mortality at 3 months was $13.6 \%$ Multivariate analysis indicated that 7-day NIHSS score was the most important predictor of 3-month mRS

Conclusion: Early appropriate therapy immediately after emergency MRI improves 7-day neurological symptoms and subsequent intensive rehabilitation improves 3 month clinical outcome. As 7-day NIHSS score is the most important predictor of 3-month clinical outcome, MRI-based appropriate therapy is important.
EPIDEMIOLOGY OF STROKE IN INDONESIA: A SYSTEMATIC REVIEW Y. Kusuma ${ }^{1}$, N. Venketasubramanian ${ }^{2}$, L. Soertidewi Kiemas ${ }^{1}$, J. Misbach ${ }^{1}$ ${ }^{1}$ Department of Neurology, University of Indonesia, Indonesia, Jakarta, Indonesia; ${ }^{2}$ Division of Neurology, National University Hospital, Singapore, Singapore, Singapore

Background: Stroke is a major cause of death and disability in many countries in the world. There have been a few publications about stroke epidemiology in Indonesia, home to 240 million people. This paper is a systematic review of publications of stroke epidemiology and its risk factors in Indonesia.

Methods: An electronic search was performed of PubMed from 1966 to $31 \mathrm{Dec}$ 2008 using search terms "cerebrovascular disease OR stroke" and "Indonesia". Abstracts describing epidemiology were reviewed. Where possible, full papers and their reference lists were also reviewed. Data on mortality, incidence, prevalence, costs and subtypes were extracted if available.

Results: In a survey of 258,366 households comprising one million people in 33 provinces, stroke was the leading cause of mortality among those aged over 5yr, comprising $15.4 \%$ of all deaths. In a population-based study of 2073 randomly selected people out of a total population of 523,000 people aged 25-64 years from 3 districts in urban Jakarta, using the MONICA Protocol, sequelae of stroke was found in $0.5 \%$, hypertension in $14.9 \%$, smoking in $59.9 \%$ of males and $5.9 \%$ of females, hypercholesterolemia (serum total cholesterol $>6.5 \mathrm{mmol} / \mathrm{L}$ ) in $13.4 \%$, regular alcohol intake in $2.7 \%$. In a survey of 2065 acute stroke patient admitted to 28 hospitals throughout Indonesia, the mean age was 58.8yr (SD 13.3, range 18-95), with more males than females. The most frequent risk factors were hypertension, diabetes, smoking and heart disease; recurrent stroke was found in $20 \%$. Motor symptoms were the most frequent. Ischemic stroke comprised $61 \%$. The majority were alive on discharge.

Conclusions: Stroke is a major cause of mortality in Indonesia. There is a high frequency of smoking and hypertension in the community. Hospitalised patients were younger than in most Western populations. Ischemic stroke is the most frequent subtype. More data is needed on the incidence and prevalence of stroke in Indonesia.

\section{Epidemiology of stroke}

\section{MULTIPLE INFARCTS INVOLVING THE POSTERIOR CIRCULATION IN} WEST CHINA

L. Xiao, H.B. Zhen, M. Yang, X. Yang, M.K. Zhou, Y.C. Wang, L. He

West China Hospital of Sichuan University, Chengdu, China

Background: Infarcts in the posterior circulation are common. Although there are several posterior circulation ischemic stroke registries and the associated clinical features are known, most studies have been limited to patients with involvement of single infarct. However, infarction may involve more than one site. Reports regarding multiple infarcts in the posterior circulation among Chinese population are scarce.

Patients and methods: We analyzed the demographic features, risk factors, clinical findings, angiographic feature, infarct distribution and discharge outcome of 34 patients in whom MRI scans showed infarcts that involved two or more sites of posterior circulation. All patients were underwent digital subtraction angiography (DSA) and MRI, enrolled from January 2001 to September 2007 in west China hospital.

Results: The mean age was 54.9 years and there were 28 men $(82.4 \%)$ and 6 women $(17.6 \%)$.Hypertension was the most common risk factors $(58.8 \%)$. Vertigo $(55.9 \%)$ was the most frequent clinical findings. The most common vascular lesions were vertebral artery origins (14), followed by intracranial vertebral artery (13) and basilar artery (7). All patients had two to four different infarcts including pons (22), thalamus (16), cerebellum (14), occipital lobe (10), temporal lobe (9), rostral brainstem (3), medulla (2). Infarcts most often included the middle the distal territories $(35.3 \%)$. The hospital mortality rate was only $2.9 \%$. Patients with simultaneous involvement of proximal, middle and distal territories or basilar artery lesions were likely to have poor outcome.

Conclusion: In this study among Chinese patients in west China, multiple infarcts in the posterior circulation usually involved the pons, and simultaneous middle and distal territory infarcts were the most common. Different patterns of infarcts distribution and vascular lesions were associated with different clinical prognosis. 


\section{Experimental studies} A STUDY ON RELEVANT FACTORS INFLUENCING MALNUTRITION IN
POST-STROKE PATIENTS

J.S. Yang ${ }^{1}$, S.S. Wang ${ }^{2}$, X.Y. Zhou ${ }^{2}$, Z.L. Chen ${ }^{2}$, C.F. liu ${ }^{1}$, Y.P. Shen ${ }^{3}$,

J.J. Hao ${ }^{1}$

${ }^{1}$ Department of Neurology of the Second Affiliated Hospital of Soochow

University, Suzhou, China; ${ }^{2}$ Department of neurology of Branch of ShangHai

First Hospital, ShangHai, China; ${ }^{3}$ Soochow University, Suzhou, China

Objective: To investigate the detection rate of malnutrition in post-stroke patients on community hospitals, and to discuss the relevant factors that influence malnutrition after stroke.

Methods: A cross sectional study was carried out on 438 post-stroke patients who were admitted in community hospitals, information was gathered about demographic characteristics, nutrition assessment indices and the relevant indices that probably induce malnutrition.

Outcome: The detection rate of malnutrition reaches $52.7 \%$ in post-stroke patients Comparing each group respectively through multivariate logistic regression analysis, we found there is higher malnutrition detection rate in the post-stroke patients with more stroke attack (group three stroke attackes and above., $0 \mathrm{R}=11.00<$ font 95\%CI:1.14-106.34), higher NIHSS score (group NHISS $\geq 15,0 \mathrm{R}=7.09,95 \% \mathrm{CI}$ 2.90-17.36), higher modified Rankin scale (group mRS 4-5, OR

$5.77,95 \% \mathrm{CI}$ and lower BI (group BI 0 6.31-63.52) (trend test $\mathrm{p}>0.0001$ ). The risk of malnutrition is also correlated with the post-stroke depression, family care, early-stage rehabilitation, malignant tumor and alcoholic.

Conclusion: There are high detection rate and many influence factors of malnutrition in post-stroke patients on community. With more attention to controllable influence factors will improve the prognosis of post-stroke patients.

Key words: stroke; malnutrition; prognosis

\section{Epidemiology of stroke}

\section{A LONG-TERM FOLLOW-UP OF STROKE PATIENTS}

M. Fudala ${ }^{1}$, W. Brola ${ }^{1}$, J. Czernicki $^{2}$

${ }^{1}$ St. Lucas Specialist Hospital, Neurological Ward with Stroke Unit, Konskie,

Poland; ${ }^{2}$ Medical University of Łódź, Clinic of Medical Rehabilitation, Łódź, Poland

Background and purpose: Although stroke is a major public health issue, little has been published on long-term stroke outcome in Poland. The objectives of this study were to determine a three-year outcome of stroke patients in terms of functional state, neurological and medical complications, treatment and lifestyle changes.

Methods: 153 patients suffering the first stroke in 2004/2005 were discharged from Neurological Ward with Stroke Unit of Specialist Hospital in Końskie (Poland). Three years later they completed an interview questionnaire including Barthel Index, modified Rankin Scale, Mini-Mental State Examination, Geriatric Depression Scale, and were grouped by gender (male/female) and age ( $<65$ years $/ \geq 65$ years). Results: The data was collected from $133(87 \%)$ patients. It was settled that 32 people died after being discharged from hospital. 101 people were examined. $\mathrm{Pa}$ tients functionally independent (Rankin $0-1$ ) made $47 \%, 42 \%$ were mildly disabled (Rankin 2-3) and $11 \%$ were severely/moderately disabled (Rankin 4-5). The most often recognized after-stroke complications were depressive disorders $(45,5 \%)$, falls $(43 \%)$, dementia (34\%), constipation (24\%), cardiological complications $(20 \%)$, pain and edema in paretic limbs $(16 \%)$, gastrological complications $(11 \%)$. In three years' time a third of the patients underwent various forms of rehabilitation. Most $(80 \%)$ of the patients consulted a district physician, hipotensional drugs were taken by $76 \%$, aspirin by $62 \%$, other antiplatelet drugs by $15 \%$, and anticoagulants by $7 \% .22 \%$ of them smoked cigarettes, $34 \%$ were overweight, $30 \%$ were physically active.

Conclusion: The functional state of stroke patients in three-year follow-up is not dependent on the patients' gender and age. There is a serious need to monitor a long-term clinical state of stroke patients, especially in case of neurologica complications with the aim of early diagnosis and treatment of these complications.

\section{Experimental studies}

ACTIVATION OF CEREBRAL PEROXISOME PROLIFERATOR-ACTIVATED RECEPTORS GAMMA UP-REGULATES THE INTERLEUKIN-1 RECEPTOR ANTAGONIST IN PERI-INFARCT CORTICAL TISSUE AFTER ISCHEMIC STROKE

Y. Zhao, T. Glatz, I. Stöck, P. Gohlke, J. Culman

Institute of Experimental and Clinical Pharmacology, University Hospital of

Schleswig-Holstein, Campus Kiel, Kiel, Germany

Background: Interleukin-1 (IL-1) plays a substantial role in the initiation and promotion of inflammation and the development of brain damage after ischemic stroke. Interleukin-1 receptor antagonist (IL-1ra) is natural agonist which inhibits the effects of IL-1 on target cells. The present study clarifies the role of cerebral peroxisome-proliferator-activated receptors gamma (PPARgamma) in the regulation of IL-1ra expression in the peri-infarct frontoparietal cortex of rats exposed to cerebral ischemia.

Methods: The selective PPARgamma agonist, pioglitazone $(3 \mathrm{nmol} / \mathrm{h})$ or vehicle (controls) were infused intracerebroventricularly (ICV) via osmotic minipumps in male Wistar rats, over a 5-day period before, and 24 or $48 \mathrm{~h}$ after transien middle cerebral artery occlusion (MCAO) for $90 \mathrm{~min}$. The expression of IL-1 beta, IL-1ra at both time points was studied by immunohistochemistry and Western blot Cortical cell cultures were used to ascertain the role of the PPARgamma in the regulation of IL-1ra expression in neurons.

Results: Activation of cerebral PPARgamma reduced the infarct size at both time points (by $37 \%$ and $32 \%$, respectively). Pioglitazone infused ICV down regulated IL-1 beta protein and decreased the number of IL-1 beta positive cells in the frontoparietal cortex adjacent to the ischemic core. Pioglitazone up-regulated IL-1ra protein in the peri-infarct cortical tissue and increased the numbers IL-1ra positive cells in this area at both time points after MCAO. In primary cortical cell culture, the selective PPARgamma antagonist, GW-9662, failed to reverse the pioglitazone-induced up-regulation of IL-1ra protein, indicating that this effect was not mediated by PPARgamma.

Conclusion: The inhibition of IL-1 beta expression und the up-regulation of IL-1ra by pioglitazone contribute to the suppression of post-ischemic inflammation in brain tissue, limits the progression of ischemic injury and improves the recovery from ischemic stroke.

\section{Experimental studies}

\section{DIFFUSION TENSOR MRI SHOWS CEREBRAL REPERFUSION INJURY IN} THE PENUMBRA IN NONHUMAN PRIMATES ISCHEMIA MODEL

J. Guo ${ }^{1}$, H.B. Zheng ${ }^{1}$, J. Yang ${ }^{1}$, W.Y. Cao ${ }^{1}$, L. Xiao ${ }^{1}$, Q.Y. Gong ${ }^{2}$, H.X. Li ${ }^{3}$, J.Q. Chen ${ }^{4}$

${ }^{1}$ Department of Neurology, West China Hospital of Sichuan University, Chengdu, China; ${ }^{2}$ Huaxi MR Research Center (HMRRC), Department of Radiology, West China Hospital of Sichuan University, Chengdu, China;

${ }^{3}$ National Chengdu Center for Safety Evaluation of Traditional Chinese Medicine, West China Hospital of Sichuan University, Chengdu, China; ${ }^{4}$ Laboratory of Transplant Engineering and Immunology, West China Hospital of Sichuan University, Chengdu, China

Background: Reperfusion after cerebral ischemic may exacerbate the brain injury, which named "cerebral reperfusion injury". Although there is little knowledge about changes in the brain's MRI parameters during this injury. This study was designed to use diffusion tensor imaging (DTI) parameters to evaluate this injury in infarct core (IC) and ischemic penumbra (IP) respectively in a rhesus transient middle cerebral artery occlusion (MCAo) model.

Methods: Using six adult male rhesus monkeys, the microcatheter was inserted into the MCA via the femoral artery to block the blood flow for 2 hours to create MCAo. DTI were performed $1 \mathrm{~h}$ after the ischemia and repeated $1 \mathrm{~h}, 3 \mathrm{~h}, 24 \mathrm{~h}$, and $7 \mathrm{~d}$ after the reperfusion. The relative apparent diffusion coefficient (rADC) and relative fractional anisotropy (rFA) in IC area (IC group), infarct growth area (IG group) and reversible penumbra area (RP group) were measured.

Results: The rADC increased in three groups in the early stage of reperfusion (1 $\mathrm{h}$ after the reperfusion). But the rate of rADC improvement was significant slower in IG than in IC and RP. Different temporal evolution of rFA could be observed in three groups in the following stage of reperfusion ( 3 to $24 \mathrm{~h}$ after the reperfusion), which continued to decline in IG but slightly elevated in IC and RP.

Conclusion: The different temporal evolution of diffusion parameters in different areas may suggest that the injury caused by the reperfusion of blood flow was mainly in IG. 
3 Experimental studies

EFFECTIVENESS OF A NEW MODIFIED KOIZUMI SUTURE FOR TEMPORARY MIDDLE CEREBRAL ARTERY OCCLUSION IN THE RAT

A. Lourbopoulos, D. Karacostas, N. Artemis, I. Milonas, N. Grigoriadis

B' Dept. of Neurology, Lab. of Experimental Neurology and

Neuroimmunology, AHEPA University Hospital, Thessaloniki, Greece

Background: Intraluminal middle cerebral artery occlusion (MCAO) is a common model of focal ischemia in the rat. However, significant suture and weight dependent variability along with increased risk of subarachnoid hemorrhage (SAH) remain significant drawbacks of the model. Our purpose was to test a modification of the Koizumi suture in an attempt to increase reproducibility and decrease $\mathrm{SAH}$ rates in the MCAO model, irrespectively of the animals' weights.

Methods: We compared a Koizumi 5/0 Ethilon poly-L-Lysine coated suture (s-2, Group B, n=40) to an identical, uncoated one (s-1, Group A, n=18) and the Belayev's 3/0 suture (s-3, Group C, $n=15$ ), in the 2-hour MCAO model in Wistar rats of varying weight (310-527grs). Model assessment included successful infarction rates, the modified Neurological Stroke Scale (mNSS), a modified Bederson's Scale (mBS), the Grid-Walking Test (GWT), infarction volume (with rostrocaudal subanalysis and analysis of cortical/striatal involvement) and hemispheric edema.

Results: The s-2 suture increased the successful MCAO from $61.1 \%$ and $66.6 \%$ (groups A and C) to $97.5 \%$ in group B, induced a more severe clinical stroke as evaluated by mNSS (mean \pm sd for groups $\mathrm{A}=5.4 \pm 4.4, \mathrm{~B}=8.0 \pm 3.9$ and $\mathrm{C}=$ 3.9 $\pm 3.6 ; \mathrm{P}=0.004$ ), GWT parameters and $\mathrm{mBS}$ (median for groups $\mathrm{A}=2.5, \mathrm{~B}=$ 5 and $\mathrm{C}=1.0 ; \mathrm{P}=0.021$ ). These clinical data corresponded to a larger infarction produced by s- 2 suture (\% infarction volume \pm sd for groups $\mathrm{A}=9.06 \pm 9.06 \%$, B $=15.06 \pm 9.79 \%$ and $\mathrm{C}=8.26 \pm 8.83 \% ; \mathrm{P}=0.024)$ with significant larger cortical ischemic lesion. Hemispheric edema was also significantly higher in group B animals $(\mathrm{P}<0.05)$. Most importantly, results of group $\mathrm{B}$ were independent to the animal's weight (linear regression, $\mathrm{P}>0.05$ ), with no incidence of SAH.

Conclusions: Our new modified suture induces a more reproducible and severe ischemic stroke model in Wistar rats for temporary-MCAO experiments, overcoming the variability of weight and the risk of SAH.

\section{Experimental studies}

THE NOVEL ANTICOAGULANT SOLULIN PROMOTES REPERFUSION AND REDUCES INFARCT YOLUME IN A THROMBOTIC MODEL OF STROKE

E.J. Su ${ }^{1}$, M. Geyer ${ }^{1}$, M. Wahl ${ }^{1}$, M. Warnock ${ }^{1}$, H. Brohmann ${ }^{2}$, K.U. Petersen ${ }^{2}$, D.A. Lawrence ${ }^{1}$

${ }^{1}$ Department of Internal Medicine, Division of Cardiovascular Medicine, University of Michigan Medical School, 7301 MSRB III, Ann Arbor, MI, USA; ${ }^{2}$ PAION Deutschland GmbH, Aachen, Germany

Background: Urgent anticoagulation is not recommended in moderate and severe strokes for lack of efficacy and a risk of intracranial hemorrhage. Solulin is a soluble recombinant analogue of the endothelial cell receptor thrombomodulin that acts by converting thrombin from a procoagulant to an activator of the anticoagulant zymogen protein C. Thus, Solulin's activity is restricted to sites with increased thrombin concentration. Previously, we found that Solulin is able to inhibit ongoing thrombosis and restore vascular patency in a murine model of acute ischemic stroke.

Methods: Photothrombotic occlusion of the middle cerebral artery of mice was induced by local photoactivation of Rose Bengal dye. Cerebral blood flow (CBF) was monitored continuously using laser Doppler analysis for $1.5 \mathrm{~h}$ after occlusion and again $72 \mathrm{~h}$ after stroke. Brain tissue was analyzed 24 or $72 \mathrm{~h}$ post-occlusion.

Results: None of the vehicle-treated mice showed significant restoration of CBF in the first $120 \mathrm{~min}$ after stable occlusion and only $17 \%$ did so at $72 \mathrm{~h}$ after stroke. Solulin $(1 \mathrm{mg} / \mathrm{kg}$ ) administered 30 or $60 \mathrm{~min}$ post-stroke restored CBF in a significant number of animals within 90 min of treatment. This effect was sustained at $72 \mathrm{~h}$ in the group treated $30 \mathrm{~min}$ post-stroke, while both treatment groups showed significant reductions of infarct size. In contrast, solulin did not influence CBF or infarct size in mice homozygous for the FV Leiden mutation, indicating that Solulin's anticoagulant activity is critical for its neuroprotective activity. Solulin was well tolerated and no overt hemorrhage was observed. Quantitative analysis of hemoglobin in brain tissue $24 \mathrm{~h}$ post-stroke showed no significant difference between control mice and mice treated with either $1 \mathrm{mg} / \mathrm{kg}$ of Solulin or $10 \mathrm{mg} / \mathrm{kg}$. Conclusion: Solulin is a safe and effective anticoagulant able to antagonize thrombosis and its sequelae in thrombotic stroke without any apparent increase in the risks of hemorrhagic complications.
5 Experimental studies

COMPARISON OF EFFECTIVENESS ON LATE STAGE OF STROKE RATS AFTER INTRAVENOUS OR INTRAPERITONEAL ADMINISTRATION OF BONE MARROW STROMAL CELLS

Y.D. Zhang, Y. Liu

Nanjing Brain Hospital, Nanjing Medical University, Nanjing City, China

Background: This study aims to investigate the therapeutic potential of adult bone marrow stromal cells (BMSCs) to cerebral ischemic rats after intraperitoneal administration by comparison with intravenous administration.

Methods: experimental rats were suffered 90 minutes of middle cerebral artery occlusion (MCAO), divided into group 1 (rats given MCAO alone without donor cell administration), group 2 (rats given BMSCs intravenously at 21 days after $\mathrm{MCAO}$ ) and group 3 (rats given BMSCs intraperitoneally at 21 days after MCAO), sacrificed at 7,14 or 21 days after treatment. BrdU was injected intraperitoneally to label BMSCs.

Results: Immunohistochemical studies suggested that transplanted cells survived and migrated to the ipsilateral cerebral cortex both after intraperitoneal and intravenous injection. Some of them were immunopositive for neuronal marker microtubule associated protein (MAP-2), astrocytic maker glial fibrillary acidic protein (GFAP) or microvessel marker Angiogenin in vivo. Evaluation with both neurological severity score (NSS) and Adhesive-removal somatosensory test showed that neurological functions were significantly improved after intravenous injection of BMSCs compared with intraperitoneal injection. The average number of neovessels measured with most conferted expression of Angiogenin was revealed that the number of neovessels in group $2(143.5 \pm 6.5)$ was significantly higher than that in group $1(107.5 \pm 3.2)(\mathrm{P}<0.01)$ and group $3(110.7 \pm 5.0)(\mathrm{P}<0.01)$ after 21 days of treatment, while there was no significant difference between control group and group 3 .

Conclusions: This study indicated that BMSCs can migrate into brain tissue to induce neuronagenesis and angiogenesis either by intraperitoneal or intravenous administration, even at the late stage of ischemic cerebral injuries, the effectiveness of intravenous injection was better.

\section{Experimental studies}

\subsection{T MAGNETIC RESONANCE SPECTROSCOPIC EVALUATION OF} METABOLIC CHANGES IN THE MOUSE STRIATUM FOLLOWING TRANSIENT MIDDLE CEREBRAL ARTERY OCCLUSION C. Berthet ${ }^{1}$, H. Lei ${ }^{2}$, R. Gruetter ${ }^{2}$, L. Hirt $^{1}$

${ }^{1}$ CHUV, Lausanne, Switzerland; ${ }^{2}$ EPFL, Lausanne, Switzerland

Magnetic resonance imaging (MRI) and spectroscopy (MRS) allow establishing the anatomical evolution and neurochemical profiles of ischemic lesions. However only limited MRS studies have been reported to-date in mice due to the challenges of MRS in small organs. The aim of the current work was to study the neurochemical and imaging sequelae of ischemic stroke in a mouse model in a horizontal bore 14.1 Tesla system.

ICR-CD1 mice were subjected to 30 minute transient middle cerebral artery occlusion. The extent of the lesion was determined by MRI. The neurochemical profile consisting of the concentrations of 22 metabolites was measured longitudinally following the recovery from ischemia at 3,8 and $24 \mathrm{~h}$ in the striatum.

Our model produced very reproducible striatal lesions which began to appear on $\mathrm{T} 2$-weighted images $8 \mathrm{~h}$ after ischemia. At $24 \mathrm{~h}$, they were well established and their size correlated with lesions measured by histology. Profound changes could be observed in the neurochemical profiles of the core of the striatal lesions as early as $3 \mathrm{~h}$ post-ischemia, in particular, we observed elevated lactate levels, decreases in the putative neuronal marker $\mathrm{N}$-acetyl-aspartate and in glutamate, and a transient two-fold glutamine increase, likely linked to excitotoxic release of glutamate and conversion to glutamine. With further ischemia evolution, other changes appeared at later time-points, mainly decreases of metabolites, consistent with disruption of cellular function. It is interesting to note that glutamine tended to return to basal levels at $24 \mathrm{~h}$.

We conclude that early changes in markers of energy metabolism, glutamate excitotoxicity and neuronal viability can be detected with high precision non-invasively in mice following stroke. Such investigations should lead to a better understanding and insight into the sequential early changes in the brain parenchyma after ischemia, which could be used e.g. for identifying new targets for neuroprotection. 
7 Experimental studies

PROTECTIVE EFFECT OF NARGININ AGAINST ISCHEMIC REPERFUSION CEREBRAL INJURY: POSSIBLE NEUROBEHAVIORAL, BIOCHEMICAL, CELLULAR AND HISTOLOGICAL ALTERATIONS IN CORTEX, STRIATUM, CEREBELLUM OF RAT BRAIN

A. Kumar, V. Gaur, A. Aggarwal

Panjab University, Chandigarh, India

Introduction: Cerebral ischemia is well known pathological condition occurs due to interruption of blood flow in the brain. The present study was conducted with an aim to explore the possible role of naringin against ischemia reperfusion induced - neurobehavioral, biochemical, cellular and histological alterations in cortex, striatum, cerebellum of rats.

Materials and methods: Male wistar rats (200-220 g) were subjected to bilateral carotid artery occlusion for $30 \mathrm{~min}$ followed by reperfusion for $24 \mathrm{~h}$ to induce reperfusion (I/R) cerebral injury. Naringin $(50,100 \mathrm{mg} / \mathrm{kg}$, i.p.) was administered for seven days continuously before subjected to Ischemia reperfusion injury. Various behavioral [locomotor activity, neurological score (inclined beam test), resistance to latetral push] and biochemical parameters (lipid peroxidation, nitrite level, reduced glutathione, superoxide dismutase and catalase activity) mitochondrial enzyme dysfunctions (Complex I, II, III and IV) and histopathological alterations were assessed subsequently in cortex, striatum, cerebellum areas of ischemic brain.

Results: Seven days naringin (50 and $100 \mathrm{mg} / \mathrm{kg}$ ) treatment significantly improved neurobehavioral alterations (improved locomotor activity, inclined beam walking and reduced resistance to lateral push) as compared to control (ischemia reperfusion). Naringin $(50 \mathrm{mg} / \mathrm{kg}$ and $100 \mathrm{mg} / \mathrm{kg}$ ) treatment significantly attenuated oxidative damage as indicated by reduced lipid peroxidation, nitric concentration, restored reduced glutathione and catalase activity in cortex, striatum, cerebellum as compared to control animals. In addition, naringin treatment restored mitochondria enzyme activities and histopathological alterations in cortex, striatum, cerebellum as compared to control.

Conclusion: Present study suggests the potential inhibitory effect of naringin against ischemia reperfusion injury in rats

\section{Experimental studies}

\section{THE C-JUN N-TERMINAL KINASE INHIBITION IN INTRACEREBRAL} HEMORRHAGE

D. Michel-Monigadon ${ }^{1}$, V. Mottier ${ }^{2}$, C. Bonny ${ }^{3}$, L. Hirt $^{1}$

${ }^{1}$ Laboratoire de Neurologie, Centre Hospitalier Universitaire Vaudois, Lausanne, Switzerland; ${ }^{2}$ Institut de Biologie et de Morphologie, Université de Lausanne, Lausanne, Switzerland; ${ }^{3}$ Xigen Pharmaceuticals, Lausanne, Switzerland

Background: In intracerebral hemorrhage ( $\mathrm{ICH}$ ), a subtype of stroke, the blood entry into the brain triggers toxicity resulting in a strong loss of neurons and inflammation. Water content is also increases leading to growing intracranial pressure, which worsens neurological outcome. C-Jun N-terminal kinases (JNKs) are activated in response to stress stimuli. Specific inhibition of JNK by a TAT-coupled peptide (XG-102) mediates neuroprotection in several models of ischemic stroke. Recently, we have noted that the JNK pathway is also activated in a mouse model of $\mathrm{ICH}$, raising the question of the efficacy of XG-102 in this model.

Method: $\mathrm{ICH}$ was induced in the mouse by intrastriatal injection of bacterial collagenase $(0,1 \mathrm{U})$. Three hours later, animals received an i.v. injection of XG-102 $(100 \mu \mathrm{g} / \mathrm{kg}$ ). The neuroscore was assessed using a scale (from 0 to 9) based on 3 behavioral tests performed daily. Then, mice were sacrificed at $6 \mathrm{~h}, 24 \mathrm{~h}, 48 \mathrm{~h}$ and $5 \mathrm{~d}$ after ICH and histological studies performed.

Results: XG-102 significantly improves neurological outcome at 24h (mean score: $1,8 \pm 1.4$ vs $3,4 \pm 1.8, \mathrm{p}<0.01$ ). Analysis of the lesion volume revealed a significant decrease of the lesion area in the treated group at $48 \mathrm{~h}\left(29 \pm 11 \mathrm{~mm}^{3}\right.$ vs $39 \pm 5 \mathrm{~mm}^{3}$, $\mathrm{p}=0.04)$. XG-102 mainly inhibits the edema component of the lesion. Indeed, a significant decrease of the brain swelling was observed in treated animals at $48 \mathrm{~h}$ $(14 \pm 13 \%$ vs $26 \pm 9 \%, \mathrm{p}=0.04)$ and $5 \mathrm{~d}(-0.3 \pm 4.5 \%$ vs $5,1 \pm 3.6 \%, \mathrm{p}=0.01)$.

Conclusions: Inhibition of the JNK pathway by XG-102 appears to lead to a significant decrease of the cerebral edema in the $\mathrm{ICH}$ model providing a furthe beneficial effect of the XG-102 treatment. This result is of interest because currently, clinical treatment for brain edema is limited. Importantly, the beneficial effects observed with XG-102 in both stroke models open the possibility to rapidly treat patients before identifying the stroke subtype by imaging.
9 Experimental studies

TERUTROBAN, A TP RECEPTOR ANTAGONIST, PROTECTS THE CEREBRAL MICROVASCULATURE BY PRESERVING THE BASAL LAMINA INTEGRITY IN A RAT MODEL OF FOCAL CEREBRAL ISCHEMIA

D. Burggraf ${ }^{1}$, J. Burk $^{1}$, M. Schneider ${ }^{1}$, V. Blanc-Guillemaud ${ }^{2}$, L. Lerond ${ }^{2}$, G.F. Hamann ${ }^{3}$

${ }^{1}$ Ludwig-Maximilians University, Munich, Germany; ${ }^{2}$ Institut de Recherches Internationales Servier, Courbevoie 92400, France; ${ }^{3}$ Department of Neurology, Dr Horst Schmidt Klinik, Wiesbaden, Germany

The effect of terutroban, a specific antagonist of the thromboxane A2/prostaglandin endoperoxide receptor (TPr), with antithrombotic, antivasoconstrictive, antiinflammatory/antiatherosclerotic properties, was investigated in rat cerebral microvasculature and brain tissue following transient ischemia.

Rats were pretreated for 2 weeks with terutroban $(30 \mathrm{mg} / \mathrm{kg} / \mathrm{d})$, aspirin $(1 \mathrm{mg} / \mathrm{kg} / \mathrm{d})$, clopidogrel $(1 \mathrm{mg} / \mathrm{kg} / \mathrm{d})$ or vehicle. The outcome was determined by a behavioura test, the infarct size and the neuronal survival. Microvascular integrity was assessed by measurement of collagen IV, microvessel number and density, haemoglobin and protein extravasation. The matrix metalloproteinase and the plasminogen-plasmin systems involved in the proteolysis of the basal lamina (BL), were also studied. Terutroban reduced infarct size by $43 \%$ (ns) along with an improvement in neurological outcome and a slight prevention in neuronal damage. Aspirin and clopidogrel had no effect on these parameters. The neuroprotective effect of terutroban stems from a microvascular protection evidenced by a significant prevention of collagen loss $(37 \%, \mathrm{p}<0.01)$ and a tendency to prevent decrease in vessel number and density. A trend towards a reduction in haemoglobin $(63 \%, \mathrm{~ns})$ and protein $(19 \%, \mathrm{~ns})$ extravasation confirmed the preserved integrity of the BL. This could be attributed to an effect of terutroban on the proteolysis. Indeed, an up-regulation of TIMP-1 (tissue inhibitor of metalloproteinase) $(30 \%, \mathrm{p}<0.001)$ and a down-regulation of uPA (urokinase plasminogen activator) $(62 \%, \mathrm{p}<0.01)$ shifted the relationship towards an increased activity of inhibitors of extracellular matrix proteolysis, with consequent protection of the microvascular BL.

In this model of focal cerebral ischemia, terutroban preserves at least in part, the consequences of brain ischemia by preserving the BL integrity and thus reduces the risk of haemorrhage. These data support its clinical use in the secondary prevention of stroke.

10 Experimental studies

ERYTHROPOIETIN AND ITS ANALOGUES IN EXPERIMENTAL STROKE: A META-ANALYSIS AND A META-REGRESSION ANALYSIS

J. Minnerup ${ }^{1}$, K. Diederich ${ }^{1}$, J. Heidrich ${ }^{2}$, A. Rogalewski ${ }^{1}$, J. Wellmann ${ }^{2}$, W.R. Schäbitz ${ }^{1}$

${ }^{1}$ Department of Neurology, University of Münster, Münster, Germany;

${ }^{2}$ Institute of Epidemiology and Social Medicine, University of Münster,

Münster, Germany

Background: Erythropoietin (EPO) was found to exert neuroprotective effects in a number of animal stroke studies. Unfortunately, the efficacy of drugs in preclinical studies does not reliably predict efficacy in stroke patients. To improve the significance of animal data systematic meta-analyses of candidate stroke drugs in animal experiments were conducted. Here we present a meta-analysis and a meta-regression analysis of EPO and its analogues in preclinical studies.

Methods: Electronic databases were searched for animal stroke studies in which the efficacy of EPO was investigated. The methodological quality of the studies was evaluated. We quantified the effect of treatment by the ratio of the mean outcome in a treatment group to the mean outcome in the control group. Outcomes within one trial were logarithmized and their variances are approximated by delta method. Percentage changes in the outcomes due to dosage and timing of treatment was estimated by applying random effects meta-regression to the logarithms of the results of each treatment and control group.

Results: 13 studies were included. No relationship between study quality and outcome was found. EPO and its analogues reduced infarct volumes by $32 \%$ $(\mathrm{P}<0.05)$ and improved functional outcome by $37 \% \quad(\mathrm{P}<0.05)$. No significant differences regarding efficacy were found between hematopoietic EPO derivates and non- hematopoietic EPO analogues. A meta-regression analysis revealed that only EPO administration within 6 after the onset of ischemia is effective.

Conclusion: This analysis further strengthens confidence in the efficacy of EPO in stroke. Also non-hematopoietic EPO analogues were highly effective which is of particularly importance since adverse effects of EPO treatment are mainly based on hematpoiesis stimulating effects. A meta-regression analysis suggests that EPO is only effective when treatment is initiated within 6 hours. 
11 Experimental studies

PREDICTABLE VENTRICULAR SHIFT AFTER FOCAL CEREBRAI ISCHEMIA IN RATS: PRACTICAL CONSIDERATIONS FOR INTRAVENTRICULAR THERAPEUTIC INTERVENTIONS A. Lourbopoulos ${ }^{1}$, D. Karacostas ${ }^{1}$, C. Simeonidou ${ }^{2}$, E. Spandou ${ }^{2}$, N. Artemis ${ }^{1}$, I. Milonas ${ }^{1}$, N. Tascos ${ }^{1}$, N. Grigoriadis ${ }^{1}$

${ }^{1}$ B' Dept. of Neurology, Lab. of Exp. Neurology and Neuroimmunology, AHEPA University Hospital, Thessaloniki, Greece; ${ }^{2}$ Dept. of Physiology, School of Medicine, Aristotle University of Thessaloniki, Thessaloniki, Greece

Background: Intracerebroventricular (ICV) administration is a reasonable route to study the direct effects of chemicals or cellular grafts in brain ischemia following temporary middle cerebral artery occlusion (t-MCAO). However, ischemic brain edema renders ICV approach difficult and uncertain due to pressure effect phenomena, particularly during the acute phase following stroke. Thus, we studied the ventricular displacement 6-18 hours after severe stroke in rats and try to set up new stereotaxic coordinates for successful ICV approach under these conditions.

Methods: Wistar rats were subjected to 2-hour t-MCAO and were clinically evaluated using the modified Neurological Stroke Scale (mNSS), modified Bederson's Scale (mBS) and Grid-Walking Test (GWT). Brains were studied at 6 and 18 hours post-occlusion for infarction volume and hemispheric edema, using TTC-staining. In addition, middle line dislocation (mlD) and stereotaxic coordinates of the lateral ventricles were studied at positions 0 and $-1 \mathrm{~mm}$ from bregma.

Results: The stereotaxic coordinates of both lateral ventricles, in the infarcted and contralateral hemisphere, significantly $(\mathrm{P}<0.01)$ changed at 6 and 18 hours after stroke, compared to those of Paxinos and Watson atlas for naïve rats. This change was linearly and highly correlated $(\mathrm{P}<0.001)$ with the mNSS, mBS and some GWT scores. Using the mNSS score and linear regression modeling we set up simple mathematical equations based on the formula "new coordinate $=$ mNSS $*$ a $+b$ ". These equations were able to estimate/calculate new, corrected, stereotaxic coordinates for ICV infusion in each individual animal and time point and increase successful ICV approach from $20 \%$ (no correction) to $80 \%$ (corrected coordinates). Conclusions: Stroke significantly changes ventricular stereotaxia in Wistar rats Thus, we propose the use of new coordinates, calculated individually for each animal by its mNSS score, for more reliable and successful ICV infusion 6-18 hours post-MCAO.

\section{Experimental studies}

\section{ACUTE HYPOXIC EFFECT ON NMDA-EVOKED CURRENTS IN RAT HIPPOCAMPAL NEURONS}

Y.Y. Lin, W.M. Yin, W.W. Zhang

Department of Neurology, Military General Hospital Beijing Command,

Beijing, China

Aim: To study the general electrophysiological characteristics of NMDA-evoked current (INMDA) and the effects of acute hypoxia on the NMDA receptor in the rat hippocampal neurons.

Methods: Whole-cell patch clamp technique was applied to record the INMDA of the NMDA receptor in primary cultured rat hippocampal neurons. An acute hypoxia model was made for neurons in vitro. All data acquired through pCLAMP8.0.

Results: NMDA $(100 \mu \mathrm{mol} / \mathrm{L})$ can evoke an inward current in primary cultured rat hippocampal neurons at a holding potential of $60 \mathrm{mV}$. The peak amplitude of INMDA is $(-745.461 \pm 123.731) \mathrm{pA}$. And the I-V relationship showed that INMDA is an inward current when the holding potential is negative while outward current when the holding potential is positive. INMDA can attenuate immediately. After acute hypoxia for $2 \mathrm{~min}$, the peak amplitude of INMDA $(-1670.49 \pm 202.09) \mathrm{pA}$ was enhanced significantly in the primary cultured neurons at a holding potential of $-60 \mathrm{mV}$ compared with the control group $(\mathrm{t}=12.572, \mathrm{p}<0.01)$.

Conclusion: INMDA displayed significant properties of inward rectification. Acute hypoxia can increase the activity of NMDA receptor in the membrane of hippocampal neurons, and NMDA receptor is involved in the excitotoxicity triggered by acute hypoxia.

\section{Experimental studies}

THE ROLE OF OCCLUDIN AND CLAUDINS IN VASCULAR DEMENTIA M.O. Romanitan ${ }^{1}$, B. Winblad ${ }^{2}$, O.A. Bajenaru ${ }^{1}$, B.O. Popescu ${ }^{1}$ N. Bogdanovic ${ }^{2}$

${ }^{1}$ Emergency University Hospital, Bucharest, Romania; ${ }^{2}$ Karolinska Institute, Alzheimer's Disease Research Center, Stockholm, Sweden

Background: Occludin and claudins are proteins of tight junctions (TJs). Occludin
(Occ) is anchoring the claudins to the cytoskeleton and plasma membranes of adjacent cells while claudin $5(\mathrm{Cl} 5)$ is expressed in the endothelial cells of all vessel segments, claudin $11(\mathrm{Cl11})$ is forming the myelin sheaths and claudin $2(\mathrm{Cl} 2)$ is a major structural component of TJ strands expressed by the epithelial cells.

The aim of this study is to analyze the expression of Occ and claudins in cerebral microvasculature and frontal cortex in control ageing brains and Vascular Dementia (VD)

Methods: The brain material -cerebral frontal cortex- was obtained from Huddinge Brain Bank, Stockholm. The study was based on 5 ageing control and 6 VD post-mortem human brains. Immunostaining with rabbit polyclonal antibody against Occ, $\mathrm{Cl}$ 2, $\mathrm{Cl} 5$ and against $\mathrm{Cl} 11$ was performed on paraformaldehyde-fixed embedded $7 \mu \mathrm{m}$ sections followed by quantitative approach using stereological principles. The neurons, astrocytes and oligodendrocytes were quantified. The stained vessels were appreciated only qualitatively.

Results: We observed the significant increase in ratio of Occ and C111 expressing neurons, glial cells or vessels in VD as compared to controls. The stained neurons were mainly pyramidal and the stained glial cells were mainly astrocytes. At the brain microvasculature level in control brains Occ was expressed in some endothelial cells while in VD the distribution was in most of the vessel wall. Claudins stained the endothelium both in white and gray matter.

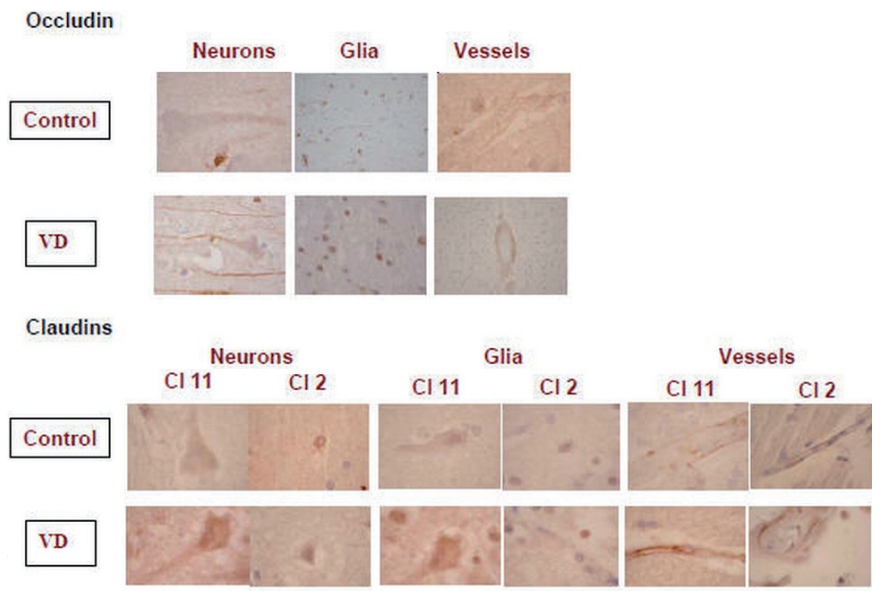

Conclusion: The study sustains the idea of occ and claudins expression in human brain neurons, glial cells and vessels suggesting that there is an increase of all protein's expression, especially occ and Cl11, in pathological conditions such as VD compared to controls. Our findings could reveal new pathogenic pathways in vascular dementia and open a new horizon for the functioning of the TJs proteins in the central nervous system.

\section{Experimental studies}

\section{STRESS AGGRAVATE THE CEREBRAL INFARCTION THROUGH THE} MITOGEN ACTIVATED PROTEIN KINASE PATHWAYS

J. Zehn ${ }^{1}$, J.W. Wu ${ }^{1}$, S.Y. Oh ${ }^{2}$, Y.H. Kim ${ }^{2}$, B.S. Shin ${ }^{2}$

${ }^{1}$ Department of Neurology, Chonbuk National University Graduate School, Jeonju, South Korea; ${ }^{2}$ Department of Neurology, Chonbuk National University Hospital and Medical School, Jeonju, South Korea

Background and aims: Conventional risk factors of cerebral infarction such as hypertension, diabetes, hyperlipidemia, and smoking are considered as independent risk factors for cerebrovascualr events. Several studies provided convincing evidence that some psychosocial factors, chronic stress and emotional factors, are independent predictors of atherosclerosis and cardiovascular events. However psychosocial factors receive little attention in the medical setting. The purpose of this study is evaluation of pathophysiologic mechanism of stress on the photothrombotic ischemic cortical injury.

Methods: Thirty male Sprague-Dawley rats were assigned to the two groups: ischemic control group $(n=15)$, ischemic treated with restrain stress $(n=15)$. Cortical photothrombosis was induced to the sensorymotor cortex and restrain stress was given during 5days. For the evaluation of behavior function, tapered/ledged beam-walking test, beam balance test, and forced swimming test were used. The infarct volume was measured by H-E stain, and the expression of P-Erk1/Erk2 was measured for the evaluation of pathophysiological mechanism.

Results: There was significant difference in cortical infarct volume between two groups $(\mathrm{P}<0.01)$. The behavior improvement has difference between two groups in 
beam-walking score and beam balance test $(\mathrm{P}<0.05)$ during 5days. The expression of P-Erk1 and P-Erk 2 were lower in stress group and the expression of P38 was increased in stress group $(\mathrm{P}<0.05)$

Conclusion: The present data showed that restrain stress on cerebral infarction effect to increasing infarction volume and decreasing functional recovery by modulation of mitogen activated protein kinase pathway.

15 Experimental studies

WITHDRAWN

16 Experimental studies

MOLECULAR MECHANISMS OF BRAIN DAMAGE RELATED WITH THE NEUROPROTECTIVE EFFECTS OF NEUROSERPIN IN PATIENTS WITH ACUTE ISCHEMIC STROKE

J. Agulla ${ }^{1}$, R. Rodriguez-Gonzalez ${ }^{1}$, O. Moldes ${ }^{1}$, J. Ferro $^{1}$, D. Brea ${ }^{1}$, S. Arias ${ }^{1}$, S. Reverte ${ }^{2}$, P. Ramos-Cabrer ${ }^{1}$, T. Sobrino ${ }^{1}$, J. Castillo

${ }^{1}$ Clinical Neuroscience Research Laboratory, Department of Neurology, Hospital Clínico Universitario, University of Santiago de Compostela, Santiago de Compostela, Spain; ${ }^{2}$ Acute Stroke Unit, Department of Neurosciences, Hospital Universitari Germans Trias i Pujol, Badalona, Spain

Background: Neuroserpin, an inhibitor of serine proteases expressed in the brain, has shown neuroprotective effects in animal models of cerebral ischemia. Our aim was to assess whether neuroserpin serum levels could be associated to biomarkers of excitotoxicity, inflammation and blood brain barrier disruption after ischemic stroke.

Methods: We prospectively included 129 patients with ischemic stroke (male $58.1 \%$; mean age, $72.4 \pm 9.6$ years), not treated with tPA within $12 \mathrm{~h}$ of symptoms onset (mean time, $4.7 \pm 2.1 \mathrm{~h}$ ). Stroke severity was evaluated by the NIHSS. Infarct volume was measured on CT performed on days 4th to 7 th. Serum levels of neuroserpin, Interleukin 6 (IL-6), Intercellular adhesion molecule-1 (ICAM1), Metalloproteinase 9 (MMP-9), and cellular fibronectin (cFn) (determined by ELISA) and glutamate (determined by HPLC) were measured on admission, 24 and $72 \mathrm{~h}$. ROC analysis was used to select the best predictive value for neuroserpin due to a lack of linearity.

Results: The decrease of neuroserpin levels within the first $24 \mathrm{~h}$ was negative correlated with serum levels at 24 hours of glutamate ( $r=-0.642)$, IL-6 ( $r=-0.678)$, ICAM-1 ( $\mathrm{r}=-0.345)$, MMP-9 $(\mathrm{r}=-0.554)$ and cFn $(\mathrm{r}=-0.703)($ all $\mathrm{p}<0.0001)$. Patient with a decrease of neuroserpin levels $<70 \mathrm{ng} / \mathrm{mL}$ at $24 \mathrm{~h}$ showed higher serum levels of glutamate, IL-6, ICAM-1, MMP-9 and cFn at 24 hours. In the multivariate analysis, the decrease of neuroserpin levels $<70 \mathrm{ng} / \mathrm{mL}$ at $24 \mathrm{~h}$ was independently associated to serum levels of glutamate (OR, 1.04; CI95\%, 1.01-1.06, $\mathrm{p}=0.001)$ IL-6 (OR, 1.4; CI95\%, 1.1-1.7, p=0.001); and cFn (OR, 1.3; CI95\%, 1.1-1.6, $\mathrm{p}=0.002$ ) after adjustment for age, gender, previous stroke, lesion volume, glucose levels and baseline stroke severity.

Conclusion: These findings suggest that neuroprotective properties of neuroserpin may be related to mechanisms of excitotoxicity, inflammation, as well as blood brain barrier disruption that occur after acute ischemic stroke.

\section{Experimental studies}

ROLE OF COLLATERAL CIRCULATION IN PROTECTIVE EFFECT OF EARLY ISCHEMIC PRECONDITIONING DURING FOCAL TRANSIENT AND PERMANENT BRAIN ISCHEMIA IN RATS

E.V. Melnikova ${ }^{1}$, A.A. Shmonin ${ }^{2}$, A.E. Baysa ${ }^{2}$, V.N. Vavilov ${ }^{2}$, T.D. Vlasov ${ }^{1}$

${ }^{1}$ Saint-Petersburg I.P. Pavlov State Medical University, Saint-Petersburg,

Russian Federation; ${ }^{2}$ V.A. Almazov Federal Centre of Heart, Blood and

Endocrinology, Saint-Petersburg, Russian Federation

Background: We hypothesize that early ischemic preconditioning (IPC) can afford protection against focal brief and prolonged cerebral ischemia with subsequent reperfusion as well as permanent brain ischemia in rats by amelioration of regional cerebral blood flow.

Methods: Adult male Wistar rats $(n=97)$ were subjected to transient (30 and 60 minutes) and permanent middle cerebral artery (MCA) occlusion. IPC protocol consisted of two episodes of 5-min common carotid artery occlusion +5 -min reperfusion prior to test ischemia either followed by 48 hours of reperfusion or not. Triphenyltetrazolium chloride and Evans blue were used for delineation of infarct size and anatomical area at risk (comprises ischemic penumbra and ischemic core), respectively. Blood flow in the MCA vascular bed was measured with use of Doppler ultrasound.

Results: IPC resulted in significant infarct size limitation in both transient and permanent MCA occlusion. Importantly, IPC caused significant reduction of area at risk after $30 \mathrm{~min}$ of focal ischemia as compared to controls [med (min-max) $11,4 \%(3,59-20,35 \%)$ vs. $2,47 \%(0,8-9,31 \%), p=0,018]$ but it failed to influence area at risk after 5 min of ischemia [med (min-max) 7,61\% (6,32-10,87\%) vs. 8,2\% $(4,87-9,65 \%), p>0,05]$. No differences in blood flow were found between IPC and control groups using Doppler ultrasound. This is suggestive of the fact that IPC does not really influence blood flow in the large cerebral arteries such as MCA but it might have some effect on smaller arteries.

Conclusion: Along with well established cytoprotective effects of IPC, IPCmediated reduction of area at risk by means of improvement in local cerebral blood flow may contribute to infarct size limitation after focal transient and permanent brain ischemia in rats.

Keywords: early ischemic preconditioning; transient and permanent focal brain ischemia; collateral circulation; cerebral blood flow.

18 Experimental studies

VIRTUAL REALITY CUES FOR GAIT IMPROVEMENT IN PATIENTS WITH IDIOPATHIC SENILE GAIT DISORDERS AND IN PATIENT WITH HISTORY OF PREVIOUS STROKES

Y. Baram, J. Aharon-Peretz

Technion - Israel Institute of Technology, Haifa, Israel

Background: Senile gait disorder is characterized by shuffling and slowness of movement. This is particularly true for older patients with previous history of strokes. Closed-loop visual feedback cues have been shown to be safer and more effective than open-loop fixed-velocity visual cues for gait improvement in Parkinson's disease patients. The effects of such cues on the walking abilities of patients with idiopathic senile gait disorder (SG) and on patients with previous history of strokes (PS) do not appear to have been studied before.

Methods: The immediate effects of visual feedback cues, provided through a portable virtual reality (VR) apparatus, on walking speed and stride length was measured in 21 randomly selected old-age home residents, including 6 patients with history of PS, with complaints of gait disturbances.

Results: Thirteen patients (13/21), including four (4/6) PS patients, improved their walking speed or stride length or both. Nine patients, including four PS patients improved their walking speed or stride length or both by more than $10 \%$. In the PS group, a marked improvement $(13.2 \pm 6.0 \%$ in walking speed and $16.6 \pm 4.7 \%$ in stride length) was noted in patients whose baseline performance was above the median. VR visual cues did not improve the gait of patients with vascular risk factors but without history of PS. Education was a relatively good predictor of improvement (e.g., for the three patients with 8 years of study, average improvement 
in walking speed was $-8.83 \pm 23.81 \%$ and in stride length $-4.67 \pm 15.30 \%$, for the four patients with 12 years of study, average improvement in walking speed was $-1.85 \pm 26.83 \%$ and in stride length $3.82 \pm 9.75 \%$, and for the two patients with 20 years of study average improvement in walking speed was $6.75 \pm 0.49 \%$ and in stride length $14.55 \pm 12.66 \%$ ).

Conclusion: VR visual-feedback cues can improve gait parameters in elderly patients with history of previous strokes. Education level is a good predictor of improvement.

\section{Experimental studies}

\section{COMBINED MECHANICAL AND STRUCTURAL CHARACTERISTICS OF CAROTID PLAQUES: ANALYSIS BY MULTI-ARRAY ECHOTRACKING SYSTEM AND MRI}

H. Beaussier ${ }^{1}$, O. Naggara ${ }^{2}$, D. Calvet ${ }^{3}$, R. Joannides ${ }^{4}$ E. Guegan-Massardier ${ }^{5}$, E. Gérardin ${ }^{5}$, M. Iacob $^{4}$, B. Laloux ${ }^{1}$, E. Bozec ${ }^{1}$, J. Bellien ${ }^{6}$, I. Masson ${ }^{1}$, C. Oppenheim ${ }^{2}$, P. Boutouyrie $^{1}$, S. Laurent ${ }^{1}$ ${ }^{1}$ Department of Pharmacology and INSERM U970, Hôpital Européen Georges Pompidou, Paris, France; ${ }^{2}$ Department of Neuroradiology, EA 4055 Centre Hospitalier Sainte-Anne, Paris, France; ${ }^{3}$ Department of Neurology, EA 4055 Centre Hospitalier Sainte-Anne, Paris, France; ${ }^{4}$ Department of Pharmacology and INSERM URM 644, Rouen, France; ${ }^{5}$ Department of Neurology and INSERM URM 644, Rouen, France; ${ }^{6}$ Department of Neuroradiology and INSERM URM 644, Rouen, France

Background: Combining functional and structural approaches may improve the predictive value for plaque rupture and ischemic events. Two distinct distensibility patterns were previously determined along the common carotid artery (CCA) (Paini et al. Stroke 2007; Beaussier et al. Hypertension 2008): Pattern A (larger radial strain at the plaque level than at adjacent CCA) and its opposite, Pattern B, which is more frequent in hypertensives. The aim of the study was to correlate arterial mechanics and composition of an atherosclerotic plaque at the site of the CCA

Method: 27 patients with carotid stenosis and an atherosclerotic plaque on the ipsilateral CCA were included: 18 asymptomatics (AS) and 9 symptomatics (S i.e. with previous ischemic stroke). Mechanical parameters were measured at 127 sites on a $4 \mathrm{~cm}$ long CCA segment by a novel non-invasive echotracking system $\left(\right.$ ArtLab $^{\circledR}$ ) and plaque composition was determined by non invasive magnetic resonance imaging (MRI).

Results: There was a trend (chi square $\mathrm{P}=0.054$ ) for pattern $\mathrm{B}$ (25 plaques) being more often associated with "complex" plaque (i.e. AHA stage IV-VII) than pattern A (21 plaques). In S patients, plaques on CCA ipsilateral to stroke were characterized by an outer remodeling $(\mathrm{P}=0.06)$ (increased external diameter and no change in internal diameter) whereas plaques in AS patients grew according to an inner remodeling $(\mathrm{P}<0.05)$. Plaques composed of lipids described an outer remodeling $(\mathrm{P}<0.005)$ whereas plaques without lipid showed an inner remodeling of the arterial wall $(\mathrm{P}<0.05)$.

Conclusion: Plaques in patients with previous ischemic stroke differed from those in asymptomatic patients: their abnormalities in mechanics (increased stiffening) and composition (outer remodeling, more complex structure and higher lipid content) may expose to a higher risk of rupture.

\section{Experimental studies}

\section{KB-R7943, A BLOCKER OF REVERSE SODIUM-CALCIUM EXCHANGE, PROTECTS AXONS FROM ACUTE ISCHAEMIA INDUCED BY ENDOTHELIN-1 FOR AT LEAST THREE DAYS IN THE RAT SPINAL CORD F. Bei, K.J. Smith \\ Institute of Neurology, University College London, London, United Kingdom}

Background: Ischaemic injury of the central white matter (IICWM) has been relatively little studied despite its clinical importance. We previously reported a new terminal model of IICWM in the rat spinal cord achieved by a combination of electrical stimulation and endothelin-1 (ET-1; $2.28 \mathrm{nmol}$ )-induced vasoconstriction Using this terminal model, we also reported that KB-R7943, a "classic" blocker of the reverse-mode operation of the sodium-calcium exchanger, improved axona conduction 4.5 hours after the injection of ET-1. Here, we test whether the protective effect of KB-R7943 persists for three days after ET-1 in rats recovered from anaesthesia.

Methods: To induce ischaemia, ET-1 (2.28 nmols) was injected bilaterally into the rat spinal grey matter, followed by a second injection of ET-1 (1.14 nmols) an hour later. KB-R7943 (30 mg/kg, i.v.) or vehicle was administered one hour before the first intraspinal injection of ET-1. Axonal conduction and the infarction area in the dorsal columns were assessed three days after ET-1 using electrophysiological and histological methods, respectively.
Results: Systemic pre-treatment with KB-R7943 significantly increased the area under the compound action potential (CAP; $76 \pm 12 \%, \mathrm{n}=8)$, compared with vehicle treatment $(51 \pm 19 \%, \mathrm{n}=8 ; \mathrm{P}<0.01)$. KB-R7943 also significantly reduced the infarction area in the dorsal columns $(56 \pm 15 \%$ for KB-R7943 vs. $78 \pm 9 \%$ for vehicle; $\mathrm{P}<0.01$ ). A strong correlation was found between the area under the CAP and the infarction area $(\mathrm{P}<0.01 ; \mathrm{r}=-0.820)$.

Conclusions: This new recovery model of IICWM may be useful for studying IICWM in vivo. Treatment with KB-7943, a blocker of reverse sodium-calcium exchange, may provide a therapeutic approach to protecting the central white matter from ischaemic injury.

\section{Experimental studies}

\section{THE ROLE OF TRYPTOPHAN CATABOLISM ALONG THE KYNURENINE} PATHWAY IN ACUTE ISCHEMIC STROKE

R. Brouns ${ }^{1}$, R. Verkerk ${ }^{2}$, T. Aerts ${ }^{3}$, D. De Surgeloose ${ }^{4}$, A. Wauters ${ }^{5}$, R. Sheorajpanday ${ }^{6}$, S. Scharpé ${ }^{2}$, P.P. De Deyn ${ }^{3}$

${ }^{1}$ University Hospital Brussels, Brussels, Belgium; ${ }^{2}$ Laboratory for Medical Biochemistry, University of Antwerp, Antwerp, Belgium; ${ }^{3}$ Laboratory for Neurochemistry and Behaviour, Institute Born-Bunge, Department of Biomedical Sciences, University of Antwerp, Antwerp, Belgium; ${ }^{4}$ Dept. of Radiology, ZNA Middelheim Hospital, Antwerp, Belgium; ${ }^{5}$ Dept. of Clinical Biology, ZNA Middelheim Hospital, Antwerp, Belgium; ${ }^{6}$ Dept. of Neurology and Memory Clinic, ZNA Middelheim Hospital, Antwerp, Belgium

Background: As part of the ischemic cascade, post-stroke inflammation contributes to cerebral damage. This partially may be mediated through upregulation of the kynurenine (KYN) pathway for tryptophan (TRP) oxidation, resulting in neuroprotective (kynurenic acid, KA) and neurotoxic metabolites, among which 3-hydroxyanthranillic acid (3-HAA). In this study, we investigated whether activity of the kynurenine pathway in acute ischemic stroke is related to initial stroke severity, long-term stroke outcome and the ischemia-induced inflammatory response, as evaluated by concentrations of C-reactive protein (CRP) in plasma.

Methods: Plasma concentrations of TRP, KYN, KA, 3-HAA and CRP were assessed in 149 stroke patients at admission ( $6.9 \mathrm{~h}$ after stroke onset), $24 \mathrm{~h}, 72$ $\mathrm{h}$ and day 7 after stroke onset. We evaluated the relation between the KYN/TRP ratio (index for TRP breakdown via the kynurenine pathway), the KA/3-HAA ratio (index for balance between neurotoxic and neuroprotective kynurenine metabolites), the KYN/LNAA ratio (index for influx of peripherally produced KYN into the brain) and (a) stroke severity (NIHSS score at admission, infarct volume), (b) stroke outcome (mRS score at month 3), (c) plasma CRP levels.

Results: KYN/TRP and KYN/LNAA, but not KA/3-HAA correlated with the NIHSS score $(\mathrm{r}=0.36 ; \mathrm{P}<.001$ and $\mathrm{r}=0.27 ; \mathrm{P}=.001)$ and with the infarct volume $(\mathrm{r}=0.26 ; \mathrm{P}=.010$ and $\mathrm{r}=0.18 ; \mathrm{P}=.048)$. Patients with poor outcome (mRS 4-6) had higher mean KYN/TRP and KYN/LNAA than patients with more favourable outcome (mRS 0-3) $(0.075 \pm 0.030$ vs. $0.050 \pm 0.015 ; \mathrm{P}<.001)$. The KYN/TRP ratio at admission correlated with $\mathrm{CRP}$ levels at admission $(\mathrm{r}=0.26 ; \mathrm{P}=.002), 24 \mathrm{~h}$ $(\mathrm{r}=0.25 ; \mathrm{P}=.005), 72 \mathrm{~h}(\mathrm{r}=0.35 ; \mathrm{P}<.001)$ and day $7(\mathrm{r}=0.34 ; \mathrm{P}<.001)$.

Conclusions: Activation of the kynurenine pathway occurs in acute ischemic stroke and correlates with more severe stroke, with poor long-term outcome and with the ischemia-induced inflammatory response.

\section{Experimental studies}

GLUTAMATE AND INFLAMMATORY CYTOKINES (IL-6, TNF-ALPHA) IN FOCAL CEREBRAL ISCHEMIA: A TRANSLATIONAL STUDY P. Martinez-Sanchez ${ }^{1}$, B. Fuentes ${ }^{1}$, M. Gutiérrez ${ }^{1}$, J. Masjuan ${ }^{2}$, M.E. Novillo ${ }^{2}$, M. Alonso de Leciñana ${ }^{2}$, J. Álvarez-Grech ${ }^{1}$, M.A. Ortega-Casarrubios ${ }^{1}$, I. Sanz-Gallego ${ }^{1}$, J. Fernández-Dominguez ${ }^{1}$, E. Diez-Tejedor ${ }^{1}$ ${ }^{1}$ Cerebrovascular Research Lab., Stroke Unit, Deptment of Neurology, La Paz University Hospital, Autonoma de Madrid University, Madrid, Spain; ${ }^{2}$ Stroke Unit, Department of Neurology, Ramon y Cajal University Hospital, Alcala de Madrid University, Madrid, Spain

Introduction: Glutamate and cytokines are involved in the pathophysiology of cerebral ischemic damage. It is not clear if glutamate is a specific biomarker of brain damage or can be increased in other pathological conditions.

Aim: to evaluate the glutamate and cytokines (IL-6,TNFa) plasma levels in 3 groups: 1) Acute cerebral infarct (CI) humans, 2) rat model of focal cerebral ischemia, 3) rat model of tissular stress.

Methods: Groups: Humans (G1): Prospective case-control study. Cases (n=58):acute CI patients;Controls $(n=19)$ :acute non-neurological diseases. Variables: glutamate, TNF receptor 1 (TNF-R1) and IL- 6 within first $12 \mathrm{~h}$ after symptoms onset and at 72h; stroke severity (NIHSS), disease severity (APACHE); CI volume; 3-months 
functional status (mRS). Embolic stroke model (G2): Long-Evans (LE) rats. Subjects $(n=5)$ : internal carotid artery embolization with autologous clot. Tissular stress model (G3): LE rats $(n=5)$ subjected to muscle compression in back paw during $180 \mathrm{~m}$. Variables (rats): glutamate, TNFa and IL-6 at 3 and $72 \mathrm{~h}$ (G2 and G3); volume of ischemic lesion (H\&E) and neuronal death (TUNEL) (G2).

Results: Human: Glutamate and cytokines were increased over time. However, they did not correlate with infarction size and tended to be higher in non-neurologica damage. A positive correlation was found between IL-6 at $72 \mathrm{~h}$ and stroke severity $(\mathrm{r}=0.543, \mathrm{P}<0.0001)$ as well as 3 -months $\mathrm{mRS}(\mathrm{r}=0.401 ; \mathrm{P}=0.004)$ in cases; and between IL-6 and APACHE score on admission in controls ( $\mathrm{r}=0.497 ; \mathrm{P}=$ 0.036). Glutamate levels did not correlate with disease severity. Rat: Glutamate and cytokines were increased over time, but did not correlate with infarct size and neuronal death. There were no differences between subjects and sham-operated rats. Moreover, G3 showed higher glutamate levels at $3 \mathrm{~h}(\mathrm{P}=0.08)$ and $72 \mathrm{~h}(\mathrm{P}=$ 0.028 ) than $\mathrm{G} 2$

Conclusion: Glutamate and cytokines are elevated in brain infarction. However, they can be even more elevated after damage in other tissues, both in human and animal models.

23 Experimental studies

\section{QUANTITATIVE EEG CHANGES IN ACUTE ISCHEMIC STROKE UNDER CONDITION OF ADDITIONAL ACTIVATION OF EITHER AFFECTED OR INTACT HEMISPHERE}

Y.A. Voronkova, N.V. Borisova, I.V. Kichuk, E.A. Petrova, V.I. Skvortsova

Russian State Medical University, Moscow, Russian Federation

Introduction: Quantitative electroencephalography (EEG) allows to measure degree of functional brain alterations and can be used for impartial estimation of functional recovery in acute stroke patients (AIS).

Purpose: to investigate dynamics of spectral EEG parameters and interhemispheric EEG coherence in patients with AIS under condition of additional activation of either affected or intact hemisphere.

Methods: Examined 45 right-handed AIS patients. 25 - were exposed to additiona activation of either affected or intact hemisphere by special neuropsychological methods. The control group - 20 non-stimulated patients. EEG recorded at days $3,7,28$. The integral EEG power index (IPI) was calculated for each hemisphere as relation of total spectral power of alpha and beta bands.

Results: In the experimental group, the most IPI changes were revealed in the left hemisphere regardless of the side of both lesion and stimulation. In right hemispheric patients, significant increase in IPI was found out under stimulation of intact left hemisphere on day 28. In left hemispheric patients (LHP), trend to IPI increase was revealed under both right and left hemispheric stimulation. In the control group, right hemispheric patients showed no significant IPI dynamics, left hemispheric - showed trend to IPI increase, what correlated with best recovery of left hemispheric functions. On day 3, rate of averaged interhemispheric coherence in alpha and theta bands was higher in LHP. By day 28, control patients with left hemispheric lesion demonstrated decrease in theta and increase in alpha and beta band coherence, whereas in patients with right hemispheric lesion there was increase in theta bands.

Conclusions: All patients showed best IPI dynamics for left hemisphere. According to coherence analysis, structure and dynamics of interhemispheric relations in IS have differences in cases of left and right hemispheric damage, that can reflect differences in cortical-subcortical interactions.

\section{Experimental studies}

EARLY BIOCHEMICAL MARKERS OF COGNITIVE DYSFUNCTION IN PATIENTS WITH ACUTE HEMISPHERIC ISCHEMIC STROKE

I.V. Ostrovtsev, G.E. Ivanova, T.T. Kispaeva, V.I. Skvortsova

Russian State Medical University, Moscow, Russian Federation

Introduction: Determination of early biochemical markers of cognitive dysfunction in acute stroke could allow to develop early preventive and therapeutic correction of. vascular dementia. The

Purpose: to find out early biochemical markers of cognitive dysfunction in patients with acute hemispheric ischemic stroke (HIS) of moderate severity.

Methods: 31 patients with HIS of moderate severity and 30 - with carotid atherosclerosis without stroke (control group). In all included patients there was no impairment of consciousness during hospitalization. Both groups have been comparable on gender, age, initial clinical parameters and on condition of their basic cognitive state. Special neuropsychological (associative, mnestic, attentional) and biochemical tests were carried out on days 7 and 21 after stroke, and twice in the control persons.
Results: Significant decrease in all parameters of attention, visual and verbal memory, and thinking were revealed in all the patients with acute stroke on day 7 ( $p<0.01$ vs. The control) and only in 13 of them (in $41.9 \%$ ) on day 21 ( $p<0.05$ vs. The control). Lasting decrease in cognitive functions until day 21 was accompanied by significant reduction in the serum level of thyroid stimulating hormone (TSH) and dihydroepiandrosterone (DEA) which lasted from the 1st till the 21st day after stroke. On day 21, the degree of cognitive dysfunction (in integral score) was correlated with the level of TSH $(r=0.55 ; \mathrm{p}<0.02)$ and DEA $(\mathrm{r}=0.47 ; \mathrm{p}<0.05)$. In 18 patients without cognitive dysfunction on day 21 after stroke there was revealed only transitory decrease in TSH and DEA levels on day 7.

Conclusions: In comparison with the control group, more than $41 \%$ of patients with primary hemispheric ischemic stroke of moderate severity demonstrated stable significant decrease in basic mental functions - attention and memory that was accompanied by significant decrease in TSH and DEA serum levels during the acute period of stroke until day 21

\section{Experimental studies}

\section{CANNABIONID AGONIST WINN55212-2 REDUCES APOPTOTIS AFTER} HYPOXIC-ISCHEMIC BRAIN INJURY IN FETAL LAMBS

D. Alonso-Alconada ${ }^{1}$, A. Alvarez ${ }^{1}$, F.J. Alvarez ${ }^{2}$, F. Goñi-de-Cerio ${ }^{1}$, M.C. Rey-Santano ${ }^{2}$, A. Caballero ${ }^{3}$, V.E. Mielgo ${ }^{4}$, I. Lara ${ }^{1}$, A. Valls ${ }^{2}$, E. Hilario ${ }^{1}$

${ }^{1}$ Dept. Cell Biology and Histology, School of Medicine, University of the Basque Country, Leioa, Spain; ${ }^{2}$ Research Unit on Pediatrics, Cruces Hospital, Barakaldo, Spain; ${ }^{3}$ Dept. of Neuroscience, School of Medicine, University of the Basque Country, Leioa, Spain; ${ }^{4}$ Research Unit on Pediatrics, Cruces Hospital., Barakaldo, Spain

Background: Perinatal cerebral hypoxia-ischemia is a major cause of neonata morbidity and mortality. Cannabinoids have shown therapeutic potential in cerebral ischemic disordes. To determine the neuroprotective capacity of the cannabinoid agonist WIN55212-2 after hypoxic-ischemic brain injury.

Methods: 22 fetal lambs at $86-92 \%$ gestation were randomly assigned to five experimental groups: one healthy group and four hypoxic-ischemic (HI) groups (three of them received WIN5521-2 at doses of 100,1 or 0.01 micrograms $/ \mathrm{kg}$ ), after injury induced by Partial Cord Occlusion (PCO) during 60min. All lambs were maintained with mechanical ventilation for 3 hours and then sacrified. Brains were perfusion-fixed and divided into different cerebral regions which were paraffin-embedded for the quantification of apoptotic cells by TUNEL method or disaggregated and immunolabelled for S-100 protein analysis by flow citometry.

Results: The histological density of TUNEL positive-cells was lower in all groups which received WIN5521-2 in comparison with HI group, showing a significant decrease in cortex, brain stem, cerebellum and basal nuclei. Moreover, animals treated with the cannabionid agonist showed higher values in the percentage of $\mathrm{S}-100$ positive cells $(\mathrm{p}<0.05 \mathrm{vs}$ HI group) in all regions studied, except in cortex. In both studies we obtained similar data between healthy and WIN55212-2 groups. Conclusion: Our results suggest that the administration of the cannabinoid agonis WIN55212-2 after hypoxic-ischemic brain injury in preterm lambs triggers a neuroprotective effect reducing the number of apoptotic cells.

Grants: UPV/EHU06/99, Basque-Government (IT-287-07/GIC07/779 and BFI07.288) and Ministry of Health (FIS06/0908).

\section{Experimental studies}

\section{ROLE OF NOGO-A IN NEURONAL SURVIVAL IN THE REPERFUSED} ISCHEMIC BRAIN

A. ElAli ${ }^{1}$, E. Kilic $^{2}$, Ü. Kilic ${ }^{2}$, Z. Guo ${ }^{2}$, C.L. Bassetti ${ }^{2}$, M.E. Schwab ${ }^{3}$

${ }^{1}$ Department of Neurology, University Hospital Essen, Germany; Department of Neurology, University Hospital Zurich, Switzerland, Essen, Germany; ${ }^{2}$ University Hospital Zurich, Zurich, Switzerland; ${ }^{3}$ Brain Research Institute, University of Zurich, Zurich, Switzerland

Background: Nogo-A is a neurite growth inhibitor, the deactivation of which enhances brain plasticity in animal models of stroke and spinal cord trauma. A clinical trial with Nogo-A antibodies is in progress. As previous studies were carried out in permanent stroke models, we recently studied the effects of Nogo-A deactivation after transient focal cerebral ischemia. Strikingly, Nogo-A-/- mice submitted to $30 \mathrm{~min}$ middle cerebral artery (MCA) occlusion revealed an increased mortality, associated with exacerbated neurological deficits.

Methods: We now evaluated the effect of an acute Nogo-A deactivation in the reperfused brain via (a) Nogo-A-/- and (b) neutralizing Nogo-A antibodies (11C7) that we delivered i.c.v. or intrastriatally immediately after or 24 hours prior to 30 min MCA occlusion. 
Results: In mice, in which Nogo-A was constitutively deleted or inhibited with $11 \mathrm{C} 7$ prior to stroke, we show that Nogo-A deactivation goes along with decreased neuronal survival. No effect of Nogo-A blockade was seen when the antibody was delivered after the stroke. Using protein expression and interaction studies we demonstrate that in the presence of Nogo-A the small GTPase RhoA is active, whereas Rac1 and RhoB are deactivated. As a consequence, stress kinase p38 and JNK1/2 activities are low and the phosphatase PTEN is inactive, resulting in the inhibition of p53-dependent death pathways. Deactivation of Nogo-A, on the other hand, deactivates RhoA, at the same time overactivating Rac1 and RhoB, the former of which activates $\mathrm{p} 38$ and JNK1/2 via direct interaction. RhoA deactivation in turn stimulates PTEN via its downstream kinase Rock2, thus inhibiting Akt and ERK1/2, initiating p53-dependent cell death.

Conclusions: In view of Nogo-A's role in promoting neuronal survival by controlling Rac1/RhoA balance, clinical trials should be aware of potential injurious effects of axonal growth-promoting therapies. Thus, Nogo-A antibodies should not be used in the very acute stroke phase.

\section{Experimental studies}

CHRONIC EXPOSURE OF ENGINEERED NANOPARTICLES FROM METALS WORSEN HEAT STROKE INDUCED BLOOD-BRAIN BARRIER DISRUPTION, BRAIN EDEMA FORMATION AND BRAIN PATHOLOGY IN THE RAT

H.S. Sharma ${ }^{1}$, A. Sharma ${ }^{2}$, D.F. Muresanu ${ }^{3}$

${ }^{1}$ University Hospital, Uppsala University, Uppsala, Sweden; ${ }^{2}$ Uppsala University Hospital, Uppsala, Sweden; ${ }^{3}$ University of Medicine and Pharmacy, Cluj-Napoca, Romania

The possibility that chronic exposure of nanoparticles may alter biological responses to heat stroke following hyperthermia and brain pathology was examined in a rat model. Engineered nanoparticles from $\mathrm{Ag}, \mathrm{Al}$ or $\mathrm{Cu}(\sim 50-60 \mathrm{~nm})$ were administered $(30 \mathrm{mg} / \mathrm{kg}$, i.p.) once daily for 1 week in young male rats. On the 8 th day these animals were subjected to experimental heat stroke. This was achieved by subjecting the animals to $4 \mathrm{~h}$ heat stress at $380 \mathrm{C}$ in a BOD incubator. In these animals stress reaction, blood-brain barrier (BBB) permeability and brain pathology were examined. Subjection of nanoparticle treated rats to heat stress showed exacerbation stress symptoms i.e., hyperthermia, salivation and prostration and exhibited greater BBB disruption, brain edema formation and brain damage compared to normal animals. This effect of enhanced brain pathology in heat stress was most marked in animals that received $\mathrm{Ag}$ nanoparticles followed by $\mathrm{Cu}$ and $\mathrm{Al}$ treatment. These nanoparticles treated animals exhibited profound cognitive and motor function deficits as seen on rota-rod performances and grid walking tests. Treatment with antioxidant compound H-290/51 either $30 \mathrm{~min}$ or $60 \mathrm{~min}$ after heat stress did not significantly attenuate hyperthermia induce brain pathology in nanoparticle treated heat stresses rats. Whereas, administration of nanowirded-H290/51 after $30 \mathrm{~min}$ or $60 \mathrm{~min}$ heat stress markedly attenuated BBB disruption and brain pathology in these groups. Our results show that chronic nanoparticles treatment exacerbates heat stroke induced brain pathology. Furthermore our observations indicate that antioxidants if delivered properly, i.e., using nano-wired drug delivery of H-290/51 could be a new promising approach to induce neuroprotection in heat stroke or related clinical situations.

\section{Experimental studies}

\section{CEREBRAL MMP EXPRESSION IS ALTERED BY ACUTE STATIN} POST-ISCHEMIC TREATMENT IN AN ANIMAL STROKE MODEL

\section{F. Vollmar, A. Alonso, K. Zohsel, M.G. Hennerici, M. Fatar}

Dept. of Neurology; Universitaetsmedizin Mannheim, University of Heidelberg, Mannheim, Germany

Background: In addition to the cholesterol-lowering effect, statins exert vascular protective effects. Amongst others long-term statin treatment is believed to reduce the expression of matrix metalloproteinases (MMPs), e.g. MMP-9. This is known to become effective in the early post-stroke reorganization and post-ischemic recovery. We studied if and how acute statin post-stroke treatment can be used to modify MMP expression in an animal stroke model.

Methods: Stroke was induced in 10 Wistar rats by permanent MCA occlusion. Group A received no treatment, group B i.p. injection of simvastatin (40 mg/daily) on day 1 to 5 post stroke. Infarcted areas were quantified by histology on day 5. MMP-2, MMP-3 and MMP-9 immunhistochemistry was performed and MMP positive cells were counted by two blinded examiners. Cell count (per microscopic field) in statin treated vs. non-treated animals and infarcted vs. non-infarcted hemispheres was compared.

Results: The 5-day statin treatment had no effect on serum levels of cholestero and lipids. Triglycerid levels in the treatment group did drop, but not significantly.
However, the infarcted area was reduced by $33 \%$ in treatment group B $(68.9 \% \pm 19 \%$ versus $46.0 \% \pm 22 \% ; \mathrm{p}<0.05)$. No change was found for MMP-2 between groups and hemispheres. MMP-3 was significantly elevated in statin treated animals in both hemispheres compared to untreated stroke controls $(2.2 \pm 0.9$ cells vs. $1.2 \pm 1.2$ in infarcted hemisphere $(\mathrm{IH})$ and $3.3 \pm 2.7$ vs. $0.5 \pm 0.5$ in non-infarcted hemisphere $(\mathrm{nIH}) ; \mathrm{p}<0.05)$. MMP-9 was significantly elevated in IH in both groups and further increased in statin treated group $(2.5 \pm 1.4$ vs. $1.0 \pm 1.0$ in $\mathrm{IH}$ and $1.6 \pm 2.2$ vs. $0.3 \pm 0.3$ in $\mathrm{nIH} ; \mathrm{p}<0.05)$

Conclusions: Five days statin treatment after stroke had a significant effect on MMP-9 expression, which was elevated in particular in the infarcted area. Additionally, statins increased MMP-3 expression in $\mathrm{IH}$ and $\mathrm{nIH}$.

\section{Experimental studies}

\section{IRON OVERLOAD INCREASES EARLY DEATH OF NEURONS DURING HYPOXIA AND LOWERS THE OPPORTUNITY TO SAVE NEURONS AFTER REPERFUSION}

N. Degregorio-Rocasolano ${ }^{1}$, J. Ponce ${ }^{1}$, V. Guirao ${ }^{1}$, N. Pérez de la Ossa ${ }^{2}$, A. Dávalos ${ }^{2}$, T. Gasull ${ }^{1}$

${ }^{1}$ Fundació Institut d'Investigació en Ciències de la Salut Germans Trias i Pujol, Badalona, Spain; ${ }^{2}$ Hospital Germans Trias i Pujol, Badalona, Spain

Background: Iron catalyzes the generation of the free radical hydroxyl which has been suggested as a possible mediator of the tissue injury in ischemia/reperfusion. High body iron stores have been associated with neurologic worsening and poor functional outcome after acute ischemic stroke in humans. In experimental stroke models iron overload increases infarct volume and edema, while iron chelators protect the brain. However, controversy exists since increased levels of total brain iron are not found in iron overloaded animals. The purpose of the present investigation is to study the effect of iron overload on the susceptibility to damage by hypoxia and reperfusion in isolated neurons.

Methods: Primary cultured neurons were incubated with iron 48 h before a 90 min period of oxygen glucose deprivation (OGD). After OGD cells were returned to normoxia in their conditioned control medium. Cell death was determined at the end of the OGD period (hypoxia period) and $24 \mathrm{~h}$ after returning to normoxia (hypoxia-reperfusion).

Results: Pretreatment with 20 and $50 \mu \mathrm{M}$ iron increased early neuronal cell death induced by OGD during the hypoxia period compared to non treated cultures. The net difference in the percentage of cell death immediately after hypoxia or $24 \mathrm{~h}$ after return to normoxia (hypoxia-reperfusion) was found to be maximum in controls, and decreased in iron pre-treated cells in a concentration-dependent manner.

Conclusion: In our model in vitro, iron overload increases early cell death during the hypoxia period and decreases the percentage of cells alive at the end of hypoxia than can be saved after reperfusion. This effect might explain the poor response to thrombolytic therapy in stroke patients with increased body iron stores.

\section{Experimental studies}

\section{HYPERACUTE EFFECTS OF STATINS ON TRANSIENT CEREBRAL} ISCHEMIA IN THE ISOLATED GUINEA PIG BRAIN

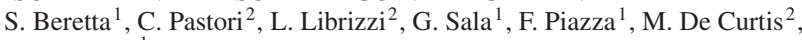
C. Ferrarese ${ }^{1}$

${ }^{1}$ Department of Neuroscience and Biomedical Technologies, University of Milano Bicocca, Monza, Italy; ${ }^{2}$ Department of Experimental Neurophysiology, Fondazione Istituto Neurologico "Carlo Besta", Milano, Italy

Background: Statins have an established role in ischemic stroke prevention and have been reported to be neuroprotective in experimental models of cerebral ischemia, possibly via Akt signalling and increased production of nitric oxide. We tested the effects of clinically meaningful doses of two chemically different statins, simvastatin (hydrophobic $100 \mathrm{nM}$ ) and pravastatin (hydrophilic $100 \mathrm{nM}$ ) on transient focal ischemia and reperfusion in the isolated guinea pig brain

Methods: This new model allows the functional preservation of both vascular and neuronal compartments, included the arteries of the circle of Willis which are perfused with a complex saline solution. Middle cerebral artery was transiently tied for $30 \mathrm{~min}$, followed by $90 \mathrm{~min}$ of reperfusion. Statins were infused continuously from 60 minutes before ischemia. Brain activity was recorded with different electrodes in brain areas within and outside MCA territory. Cerebral vascular tone was measured by changes in resistance to perfusion pressure under constant flow. The Griess assay for nitrite production was used as a marker of nitric oxide production in the venous outflow. At the end of reperfusion, tissue samples were assayed for phosphorylated Akt and Erk protein, total Akt and Erk protein and lipid peroxidation, as a marker of oxidative tissue damage.

Results: Anoxic depression in ischemic areas, vascular tone and nitrite levels in 
venous outflow were unchanged by statin treatment. Nonetheless, an activation of Akt signalling and decreased tissue oxidative damage was observed for both statins. Conclusion: Our findings suggest that statins are unlikely to exert an hyperacute effect on cerebrovascular vessels, but they may acutely protect brain tissue by counteracting oxidative damage and enhancing survival signalling.

\section{Experimental studies}

\section{SYSTEMIC MRNA EXPRESSION OF CHEMOKINE RECEPTOR 5 AND ITS LIGANDS IN ACUTE ISCHEMIC STROKE - A PILOT STUDY \\ I. Markaki, N. Kostulas \\ Division of Neurology, Neuro-Angiological Research Center, Karolinska Institutet, Karolinska University Hospital, Huddinge, Sweden, Stockholm, Sweden}

Background: Inflammation plays an important role in the pathogenesis of atherosclerosis and is an independent risk factor for stroke. Chemokines and their receptors are key mediators of inflammation. Among those, RANTES and MIP-1 alpha, ligands of chemokine receptor 5 (CCR5) are involved in inflammatory and tissue injury responses, in experimental brain ischemia. We investigated systemic CCR5 mRNA and its ligands up to one month after acute ischemic stroke (AIS).

Methods: 16 patients with AIS confirmed by computed tomography, withou earlier inflammatory conditions, were investigated. Total RNA was extracted from peripheral blood samples drawn at 1, 7 and 30 days after symptom onset. CCR5 mRNA expression was measured with real-time PCR

Results: Blood samples from nine men and seven women of a mean age $72 \pm 9$ years were analyzed. According to the Trial of Org 10172 in Acute Stroke Treatment -criteria, the distribution of the etiological subtypes was as follows: four patients with large artery disease, three with cardioembolism, four with small vessel disease and five with AIS of unknown etiology. Preliminary data showed similar CCR5 mRNA expression at all investigated time points, with a mean of $0.84 \pm 0.7$, $0.88 \pm 0.6$ and $0.82 \pm 0.6$ at day 1,7 and 30 , respectively.

Conclusion: CCR5 mRNA expression in peripheral blood cells remains unaltered within one month post AIS. The previously reported association of $\mathrm{CC}$ chemokines in AIS might represent a local inflammatory response rather than a systemic response. However, further studies are ongoing in a larger number of patients, and during an extended observational period.

\section{Experimental studies}

BRAIN AND PLASMA BDNF PROTEIN LEVELS AFTER STROKE IN RATS Y. Béjot ${ }^{1}$, C. Mossiat ${ }^{1}$, M. Giroud ${ }^{2}$, C. Marie ${ }^{1}$

${ }^{1}$ Laboratoire INSERM U887 Motricité-Plasticité, Université de Bourgogne,

Dijon, France; ${ }^{2}$ Department of Neurology, University Hospital, Dijon, France

Background: Brain-derived neurotrophic factor (BDNF) appears to be a promising target for therapeutic interventions in stroke. We aim to investigate whether stroke severity influences BDNF levels in the brain and whether a correlation exists between brain and plasma BDNF levels after stroke.

Methods: Ischemic stroke was induced in rats by embolization of the lef hemisphere with calibrated microspheres. The whole left hemisphere, differen structures of the right hemisphere as well as plasma were collected in control $(n=7)$, sham-operated $(n=5)$, and ischemic rats $(n=30)$ that were euthanized $4 h(n=10)$, $24 \mathrm{~h}(\mathrm{n}=10)$ or $8 \mathrm{~d}(\mathrm{n}=10)$ after brain embolization. BDNF levels were measured by the ELISA method.

Results: Brain BDNF levels were similar between control and sham-operated rats for the whole hemisphere (Mean of pooled values $32.5 \pm 2.3 \mathrm{pg} / \mathrm{mg}$ of proteins). The embolized hemisphere exhibited high BDNF levels from $4 \mathrm{~h}(+62 \%)$ to $8 \mathrm{~d}$ after embolization $(+25 \%)$ with a peak values at $24 \mathrm{~h}(+170 \%)$ as compared to baseline values. In the unlesioned hemisphere, modest and time-dependent changes in BDNF levels (decrease or increase) were observed in the thalamus, the striatum and the cortex, though not in the hippocampus. Pre-ischemic BDNF levels in plasma showed high inter-individual variability $(24.9 \pm 28.3 \mathrm{pg} / \mathrm{ml}, \mathrm{n}=41)$ and ischemia led to inconsistent effects on plasma BDNF levels. Finally, neither brain nor plasma BDNF levels correlated with the degree of embolization, and plasma BDNF did not correlate with brain BDNF.

Conclusion: In our stroke rat model, stroke-induced high BDNF levels in the lesioned hemisphere are independent of stroke severity, and plasma BDNF levels do not reflect brain BDNF levels after stroke.
NEUROPROTECTIVE EFFECTS OF LIPOIC ACID AND SUPEROXIDE DISMUTASE IN THE RAT MODEL OF CEREBRAL ISCHEMIA

E.V. Melnikova ${ }^{1}$, A.A. Shmonin ${ }^{2}$, N.M. Paramonova ${ }^{1}$, I.V. Churilova ${ }^{1}$ T.D. Vlasov ${ }^{1}$, E.V. Protsenko ${ }^{1}$, D.V. Gerasimenko ${ }^{1}$

${ }^{1}$ Saint-Petersburg I.P. Pavlov State Medical University, Saint-Petersburg, Russian Federation; ${ }^{2}$ V.A. Almazov Federal Centre of Heart, Blood and Endocrinology, Saint-Petersburg, Russian Federation

Background: the present study was aimed at investigation of neuroprotective effects of alpha-lipoic acid (LA) and superoxide dismutase (SOD) in brain ischemia in rats.

Methods: three models were used to produce brain ischemia: focal ischemia (permanent left middle cerebral artery occlusion), forebrain ischemia (180-min occlusion of both common carotid arteries - CCA) and permanent ligation of both CCA without subsequent reperfusion. $\mathrm{Cu} / \mathrm{Zn}-\mathrm{SOD}$ at a dose $40 \mathrm{mg} / \mathrm{kg}$, i.v. and LA at a dose of $20 \mathrm{mg} / \mathrm{kg}$, i.p. were injected 30 minutes prior or 5 minutes after onset of ischemia. The end-points of the study were histochemically determined infarct size, ultrastructural changes in the cerebral tissue, and survival rate.

Results: LA administration 30 minutes prior to ischemia dramatically reduced infarct size $(\mathrm{p}<0,001)$. Injection of LA 5 minutes after beginning of ischemia did not affect infarct size. Besides, infarct size was unchanged after injection of SOD 30 minutes prior to ischemia. On electron microscopy, increased number of tight and gap junctions was observed in ischemic area in comparison to intact brain tissue. The use of LA and SOD did not influence the number of tight and gap junctions. Electron microscopy showed that treatment with LA was associated with improved morphological appearance of blood-brain barrier as evidenced by better integrity of endothelium and basal membrane, proliferation of astroglia, and preservation of the structure of some neurons. In group where SOD was used, greater extent of injury to brain tissue was found as compared to controls. Survival rate after CCA ligation was increased in the group of LA-treated animals; in contrast, it was decreased after SOD administration.

Conclusion: the LA treatment regimen used in this study resulted in significant cerebral protection against ischemia. In contrast, SOD did not show any protective effects in focal and forebrain ischemia.

\section{Experimental studies}

THE IMPACT OF 60KHZ ULTRASOUND WITH DIFFERENT DUTY CYCLES ON ISCHEMIC BRAIN TISSUE AND THE AUDITORY SYSTEM H. Kuntze ${ }^{1}$, J. Masomi ${ }^{2}$, P. Reuter ${ }^{2}$, I. Fischer ${ }^{3}$, J.J. Marx ${ }^{1}$, K. Helling ${ }^{3}$, O. Kempski ${ }^{2}$, M. Nedelmann ${ }^{4}$

${ }^{1}$ Department of Neurology, Johannes Gutenberg-University of Mainz, Mainz, Germany; ${ }^{2}$ Institute of Neurosurgical Pathophysiology, Johannes Gutenberg-University of Mainz, Mainz, Germany; ${ }^{3}$ Department of Otolaryngology, Head and Neck Surgery, Johannes Gutenberg-University of Mainz, Mainz, Germany; ${ }^{4}$ Department of Neurology, Justus Liebig-University of Giessen, Giessen, Germany

Object: Both the efficacy and safety of therapeutic ultrasound depends on a variety of technical parameters. A recent study could demonstrate an enhanced thrombolytic activity of low frequency ultrasound in dependency of long lasting duty cycles in vitro (Meunier et al., 2007). Therefore we sought to determine the effects of varied duty cycle settings on ischemic brain tissue in a middle cerebral artery occlusion model and on the auditory system in vivo.

Methods: Male Wistar rats were assigned to undergo cerebral ischemia by the suture model for middle cerebral artery occlusion or sham operation following transcranial treatment with $60 \mathrm{kHz}$ ultrasound. Duty cycles were set at $20 \%$ or $80 \%$ while keeping time average intensity constant. Outcome assessment consisted of histology (TTC staining after 24hours) and clinical evaluation. In an additional setting healthy rats were submitted to the same ultrasound treatment in order to quantify auditory impairment by acoustically evoked potentials

Results: Ischemic lesion volume was significantly increased in animals insonated with the short $20 \%$ duty cycle setting compared to the control group whereas these side effects could not be displayed in prolonged $80 \%$ on-phase pulsation. Beyond ultrasound treatment resulted in hearing loss with a decline of the auditory threshold level up to $40 \mathrm{~dB}$.

Conclusions: Beneath the known positive impact of prolonged duty cycles on thrombolysis this study indicates a significant reduction of collateral side effects. Furthermore we could quantify for the first time ultrasound induced auditory impairment which may be a limiting factor for the application of therapeutic ultrasound. Our results may help to find a therapeutic window for future prototype ultrasound devices. 
S100BB PROTEIN SERUM LEVEL AS A MARKER OF INFLAMMATORY PROCESS DURING ACUTE ISCHEMIC STROKE

J. Bielewicz ${ }^{1}$, J. Kurzepa ${ }^{2}$, H. Bartosik-Psujek ${ }^{1}$, Z. Stelmasiak ${ }^{1}$

${ }^{1}$ Department of Neurology, Medical University of Lublin, Lublin, Poland;

${ }^{2}$ Department of Biochemistry and Molecular Biology, Medical University of

Lublin, Lublin, Poland

Background: The marker of glial cells damage, S100BB protein, have been implicated in a variety of intracellular and extracellular functions. Many research have investigated S100BB protein only as a marker of cell damage. We assumed that due to the pleiotropic functions, S100BB protein can be considered also as a marker of inflammatory response occurred within an ischemic focus. The purpose of our study was the evaluation of relationship between S100BB and well-known inflammatory marker, c-reactive protein (CRP), during acute ischemic stroke (IS). Methods: The final study group consisted of 53 patients (mean age: $72.3 \mathrm{y} \pm 11.7$; female: 29, etiology of IS, cryptogenic: 26 , atherogenic: 14, cardiogenic: 13) with CT-scan confirmed IS. Blood samples were obtained during the first $24 \mathrm{~h}$ of stroke and on days 3, 5 and 10 after symptoms onset. Commercially available ELISA kits were applied to evaluate S100BB and CRP levels.

Results: Serum S100BB level decreased gradually from day 3 till day 10 of stroke (median values: $52.9 ; 54.8 ; 52.4 ; 35.9$ on days $1,3,5$ and 10 respectively, $\mathrm{p}<0.05$ between 3 and 10 day). The median values of CRP was increasing during all evaluated time-points $(8,6 ; 17,2 ; 20,3 ; 20,4$ on days $1,3,5$ and 10 respectively, $p<0.05$ between 1 and 5 day). Statistically significant correlations between S100BB and CRP serum levels were observed at all evaluated days but the highest correlation coefficient was noticed between average of S100BB and CRP serum levels $(r=0.59$, $\mathrm{p}<0.001)$.

Discussion: S100BB protein derives from astroglial and can be detectable in blood as a result of activation or destruction of glial cells caused by ischemia. The augmentation of S100BB and CRP levels in the blood during IS can be an independent process, but the increase of S100BB serum level prior to CRP suggests that $\mathrm{S} 100 \mathrm{BB}$ contributes to the intensity of inflammatory processes and results in CRP enhancement.

\section{Experimental studies}

\section{THE IMPACT OF PETHIDINE ON THE NEUROPROTECTIVE EFFECTS OF} HYPOTHERMIA

E.S. Sena ${ }^{1}$, A.L. Jeffreys ${ }^{2}$, S.F. Cox ${ }^{2}$, L. Churilov ${ }^{3}$, S.A. Sastra ${ }^{2}$, M.R. Macleod ${ }^{4}$ D.W. Howells ${ }^{2}$

${ }^{1}$ University of Edinburgh, Edinburgh, United Kingdom; ${ }^{2}$ University of Melbourne, Melbourne, Australia; ${ }^{3}$ National Stroke Research Institute, Melbourne, Australia; ${ }^{4}$ University of Edinburgh, Edinburgh, Australia

Background: Hypothermia is a promising treatment for acute stroke. Shivering patients may be given pethidine, but any modulating effects of pethidine on hypothermia are not known.

Methods: Male spontaneously hypertensive rats were anaesthetised (isoflurane) and randomly assigned to normothermia $\left(37.4^{\circ} \mathrm{C}\right.$ rectal temperature); hypothermia $\left(33^{\circ} \mathrm{C}\right)$; pethidine $(2.5 \mathrm{mg} / \mathrm{kg})$; or hypothermia plus pethidine. Physiological parameters were recorded. Temporary (1.5hrs) filament MCAO was induced blinded to treatment allocation and was confirmed with laser doppler flowmetry (LDF). Pethidine and cooling were given at vessel occlusion. After 130 minutes animals were actively warmed. Animals in the normothermia group had active temperature management using heating lamp and fan. Animals with abnormal physiological variables or insufficient LDF drop were excluded. Animals were sacrificed at 24 hours and infarct size $\left(\mathrm{mm}^{2}\right.$, TTC staining) measured blinded to treatment group allocation. To give $80 \%$ power to detect a $40 \%$ difference using 2 way ANOVA at $\mathrm{p}<0.05,15$ animals per group were required. An independent statistician conducted a blinded interim analysis after 72 animals.

Results: Of 72 rats randomised 13 were excluded. Hypothermia reduced infarct size from $51.6(95 \% \mathrm{CI} 10.5-52.2)$ to $31.3(10.5-52.2)(\mathrm{p}=0.001)$. Pethidine increased infarct size to $90.9(70.7-111.0)(\mathrm{p}=0.029)$. In the presence of pethidine, hypothermia reduced infarct size to $37.4(17.3-57.6)(\mathrm{p}<0.05 \mathrm{v}$ normothermia, ns v hypothermia alone). The interim analysis suggested that to confirm that pethidine reduced the protective effect of hypothermia by $20 \%$ would require a further 312 animals.

Discussion: While there was no significant impact of pethidine on the protective effect of hypothermia, these data are consistent with a substantial blunting of that protection.
37 Experimental studies

INFLUENCE OF PRESSURE AND FLOW VELOCITY ON THROMBOLYSIS IN-VITRO

F.C. Roessler ${ }^{1}$, M. Ohlrich ${ }^{1}$, M. Schmieger ${ }^{2}$, P.-K. Weber ${ }^{2}$, G. Seidel ${ }^{1}$

${ }^{1}$ Clinic for Neurology, University Hospital of Schleswig-Holstein, Campus Lübeck, Lübeck, Germany; ${ }^{2}$ Fraunhofer Institute for Biomedical Engineering IBMT, Ultrasound Systems Development, St. Ingbert, Germany

Background: To examine the influence of flow, pressure, ultrasound and thrombolytic drugs on sonothrombolysis we developed a computer assisted in-vitro model, which unlike to all so far known experimental setups, is able to generate defined, pulsatile flow conditions. In this series of experiments we examined the influence of pressure and flow velocity on clot dissolution.

Method: A computer-controlled pump excites pulsatile flows in a tube-system filled with blood substitute ensuring continuously variable pulse frequencies and flow amplitudes. A blood clot is tied in a custom-build clot carrier within the tube-system. To adjust pressure and flow velocity continuously needle valves are used. The measured pressure decline at the clot carrier is directly proportional to the flow resistance and therefore to the site of the clot. Three hemodynamic adjustments $(\mathrm{A}-\mathrm{C}$ ) were compared (each $\mathrm{n}=5$ ) to investigate their impact on clot decomposition. A: Constant flow velocity $\mathrm{V}=29 \mathrm{~cm} / \mathrm{s}$, pressure before the clot $\mathrm{P} 1=30 \mathrm{mmHg}$. B: Constant V $=64 \mathrm{~cm} / \mathrm{s}, \mathrm{P} 1=130 \mathrm{mmHg}$. C: Physiological, pulsatile V, mean value of $\mathrm{P} 1 \mathrm{mP} 1=130 \mathrm{mmHg}$.

Results: After 4 hours the mean change of pressure decline at the clot varied considerably between the adjustments. A: $0.5 \pm 0.33 \mathrm{mmHg}$. B: $-0.76 \pm 0.58 \mathrm{mmHg}$ $\mathrm{C}:-4.41 \pm 0.65 \mathrm{mmHg}$. The statistical analysis of the continuously recorded data revealed significant effects between the adjustments $A / B(p=0.01), C / B(p=0.01)$ and $\mathrm{A} / \mathrm{C}(\mathrm{p}=0.1)$ at different periods.

Conclusion: The in-vitro model allows the defined adjustability of all hemodynamic parameters affecting sonothrombolysis under physiological flow conditions and permits non-contact and continuous measurements. This first experiments display the dependency of thrombolysis on the chosen flow pattern and pressure values.

38 Experimental studies

CEREBRAL MMP EXPRESSION IS NOT ALTERED BY TRANSCRANIAL ULTRASOUND AND MICROBUBBLES TREATMENT IN A HEMORRHAGIC STROKE MODEL

M. Fatar, F. Vollmar, M. Stroick, A. Alonso, K. Zohsel, M.G. Hennerici, S. Meairs

Dept. of Neurology, Universitaetsmedizin Mannheim, University of Heidelberg, Mannheim, Germany

Background: MMP expression in ischemic stroke is associated with secondary hemorrhage and brain edema formation. To examine safety of transcranial ultrasound (US) with microbbubbles (MB) we studied MMP expression in a hemorrhagic stroke rat model under US+MB treatment.

Methods: Intracranial hemorrhage (ICH) was induced in anesthetized rats by intracerebral collagenase injection. Transcranial ultrasound $(2 \mathrm{MHz}$, mechanical index $1.3,1051 \mathrm{kPa}$ ) was applied $3 \mathrm{~h}$ after ICH induction to rat brains for 30 min during a continuous IV infusion of sulfur hexafluoride MB (SonoVue ${ }^{\mathrm{TM}}$ ). $24 \mathrm{~h}$ after stroke the size of cerebral hemorrhage, the extent of brain edema, the amount of apoptosis and the number of cells (per microscopic field) stained positive for MMP-2, MMP-3 and MMP-9 were compared with those from control rats with ICH but without US+MB treatment.

Results: We found no significant effect of US+MB on hemorrhage size (US+MB $20.3 \pm 5.8 \mathrm{~mm}^{3}$ vs. control $23.3 \pm 10.7 \mathrm{~mm}^{3}$ ), on the extent of brain edema (US+MB $3.5 \pm 1.9 \%$ vs. control $3.3 \pm 2.0 \%$ ) or on the rate of apoptosis (US+MB $5.2 \pm 1.0 \%$ vs. control $5.2 \pm 1.5 \%$ ). Concerning MMP expression, no significant differences between US/MB treated group vs. control group were observed for MMP-2 (US+MB $1.8 \pm 1.5$ vs. control $3.5 \pm 4.2$ ) and for MMP-9 (4.0 \pm 5.6 vs. $2.6 \pm 3.8)$. A tendency towards higher MMP-3 expression was found in US+MB treated animals $(3.0 \pm 2.5$ vs. $0.5 \pm 0.8 \mathrm{p}=0.07)$.

Conclusion: US/MB treatment did not influence MMP expression significantly in hemorrhagic stroke and might therefore be applicable in ICH without increasing risk of brain edema or hemorrhage volume. 
INITIAL EXPLORATION ON THE CONSTRUCTION AND EVALUATION OF A RHESUS MODEL OF MIDDLE CEREBRAL ARTERY ISCHEMIA AND REPERFUSION

J. Yang ${ }^{1}$, H.B. Zheng ${ }^{1}$, J. Guo ${ }^{1}$, L. He ${ }^{1}$, W.Y. Cao ${ }^{1}$, Q.Y. Gong ${ }^{2}$, H.X. Li $^{2}$, J.Q. Chen ${ }^{2}$

${ }^{1}$ Department of Neurology, West China Hospital of Sichuan University, Chengdu, China; ${ }^{2}$ Huaxi MR Research Center (HMRRC), Department of Radiology, West China Hospital of Sichuan University, Chengdu, China

Background: Animal models of cerebral ischemia form the basis of stroke study. Nonhuman primates are the closest to humans in terms of lineage and their middle cerebral artery (MCA) ischemia-reperfusion responses are the most similar to the human ischemic stroke. Previous nonhuman primate stroke models had limited behavioral testing and imaging, and focused on the short-term outcome.

The purpose of this study was to construct and evaluate a rhesus model of MCA ischemia-reperfusion injury using the microcatheter embolization method.

Methods: Adult male rhesus monkeys $(n=3)$ were selected for this study. After the anesthesia, the microcatheter was inserted into one side of the MCA via the femora artery to block the blood flow, thereby resulting in middle cerebral artery occlusion (MCAO). Subsequently, the microcatheter was withdrawn after $2 \mathrm{~h}$ to restore the MCA blood flow and generate the ischemic reperfusion. During the study process, the animal model was evaluated using cerebral angiography, magnetic resonance imaging (MRI), and neurological examinations.

Results: The results of the angiography and magnetic resonance angiography (MRA) confirmed the occlusion and reopening of the MCA. The results of MRI suggested the existence of certain ischemic brain lesions and the neurological exam showed sustained functional deficits after the surgery.

Conclusion: The construction of the rhesus MCA ischemia-reperfusion model using the microcatheter embolization method has a series of advantages including the ease of the operation, good reproducibility, and high animal survival rates. In addition, the scope and degree of ischemic damage are easily controlled. Furthermore, this nonhuman primate cerebral ischemia-reperfusion model is especially appropriate for MRI studies of ischemia in its ultra-early stages.

\section{Experimental studies}

SONICATED AND EMULSIONED ALBUMIN MICROBUBBLES: EXTENDING THE DIAGNOSTIC UTILITY OF SONOGRAPHY

M. Spinelli ${ }^{1}$, C. Demitri ${ }^{2}$, M. Bacigaluppi ${ }^{3}$, C. Foglieni ${ }^{4}$, M. Piscopiello ${ }^{5}$, G. Giacalone ${ }^{1}$, A. Sannino ${ }^{2}$, S. Pluchino ${ }^{6}$, G. Martino ${ }^{6}$, F. Minicucci ${ }^{1}$,

M. Sessa ${ }^{1}$, G. Comi ${ }^{1}$, F. Corea $^{1}$

${ }^{1}$ Dept. of Neurology, Institute of Experimental Neurology IRCCS San Raffaele, Milan, Italy; ${ }^{2}$ University of Lecce, Dept. of Engineering for Innovation, Lecce, Italy; ${ }^{3}$ Neuroimmunology Unit, Institute of Experimental Neurology and Dept. of Neurology IRCCS San Raffaele, Milan, Italy; ${ }^{4}$ Dept. of

Cardio-Thoracic-Vascular Medicine and Surgery, CCVB Research Center, San Raffaele Scientific Institute, Milan, Italy; ${ }^{5}$ Dept. of Neurology, Institute of Experimental Neurology and Dept. of Cardio-Thoracic-Vascular Medicine and Surgery, CCVB, Milan, Italy; ${ }^{6}$ Neuroimmunology Unit, Institute of Experimental Neurology IRCCS San Raffaele, Milan, Italy

Background: Ultrasound Doppler sonography represents a quick and efficient method to identify atherosclerotic pathology of the supraaortic trunks. Despite major technological improvements of sonography over the last 20 years, up to now atherosclerotic plaque vulnerability and the risk of intraplaque haemorrhage cannot be properly studied with this technique. Ultrasound contrast agents (UCA) consist of small, less than $10 \mu \mathrm{m}$ in diameter, bubbles made of shell of albumin, surfactants or phospholipids and filled by gas, usually air or a perfluoro gas. By their physicochemical properties UCA improve the visualization of the near-wall carotid IMT. Also in the transcranial doppler UCA increase the chance to diagnose an intracranial stenosis in the absence of an optimal acoustic window. UCA also consent to visualize the neovascularization within the carotid plaque potentially overcoming current diagnostic limitations of sonography.

Aim of our study is to obtain a ready available and inexpensive UCA to improve current limitations of diagnostic sonography.

Methods: A ready-to-use UCA was obtained following the description of Feinstein et al. $20 \%$ human albumin is sonicated at $100 \mathrm{~W}$ for 1 minute and emulsionated with a high-speed homogenizer operating at $15.000 \mathrm{rpm}$ after addition of a solvent. Results: Examination at bright field microscopy $(400 \times$ magnification) and at scanning electron microscopy (SEM) revealed the presence of a large quantity of 3 $4 \mu \mathrm{m}$ microbubbles (MB) that were compared with a commercial available UCA, Optison $^{\circledR}$, Bracco, Italy. The small sizes facilitate transpulmonary passage of the $\mathrm{MB}$

Poster Session Red

Cerebrovasc Dis 2009;27(suppl 6):1-241
Conclusions: the application of this home-made UCA in the daily clinical routine may improve sonographic imaging of atherosclerotic plaques. Further analysis to evaluate the stability of the $\mathrm{MB}$ and the qualitative improvement of the echographic image with the home-made UCA within a phantom reproducing the acoustic proprieties of human carotid plaques will be performed.

\section{Experimental studies}

\section{THERAPEUTIC EFFECT OF POST-ISCHEMIC HYPOTHERMIA INITIATION} ON CEREBRAL ISCHEMIC INJURY

H. Zhang ${ }^{1}$, M. Zhou ${ }^{2}$, E.-T. Tong ${ }^{3}$

${ }^{1}$ Department of Neurology, Zhongnan Hospital of Wuhan University, Wuhan, China; ${ }^{2}$ Department of Food Science and Technology, School of Agriculture and Biology, Shanghai Jiaotong University, Shanghai, China; ${ }^{3}$ Department of Neurology, Union Hospital of Tongji Medical College, Huazhong University of Science \& Technolog, Wuhan, China

Objective: To study the efficacy of mild brain hypothermia beginning at different time intervals on cerebral ischemic injury.

Method: Male Sprague-Dawley rats were divided into a sham-operated group, normothermia $\left(37-38^{\circ} \mathrm{C}\right)$ ischemia and mild hypothermia $\left(31-32^{\circ} \mathrm{C}\right)$ groups. The last group was subdivided into four groups: 240 minute hypothermia, 30 minute normothermia plus 210 minute hypothermia, 60 minute normothermia plus 180 minute hypothermia, and 90 minute normothermia plus 150 minute hypothermia $(n=8)$. Global cerebral ischemia was established using the Pulsinelli four-vessel occlusion model for 20 minute. Mild hypothermia was apllied after 20 minute cerebral ischemia. Brain tissue were collected following a 20 minute cerebral ischemia and 240 minute reperfusion, and used to measure the levels of superoxide dismutase (SOD), glutathione peroxidase (GSH-Px), glutathione $(\mathrm{GSH})$, glutathione $(\mathrm{GSH})$, adenosine triphosphate (ATP).

Results: Mild hypothermia beginning at 0-60 minute delayed the consumption of superoxide dismutase, glutathione peroxidase, glutathione, glutathione, adenosine triphosphate $(\mathrm{p}<0.05$ or $\mathrm{p}<0.01)$ in ischemic brain tissue. Mild hypothermia beginning at 90 minute had little effect on the levels of targeted molecules in ischemic brain tissue as compared to the normothemia ischemia group ( $p>0.05)$. Discussion: Post-ischemic mild brain hypothermia can significantly delay consumption of endogenous antioxidant enzyme and energy metabolism, which is involved in the mechanism of cerebral protection by mild hypothermia. These results showed that mild hypothermia attenuated ischemic injury beginning within 60 minute, but lost ist neuroprotection effects when beginning at 90 minute following cerebral ischemia.

\section{Experimental studies}

CORRELATION BETWEEN BRAINSTEM AUDITORY EVOKED POTENTIALS, ELECTRONYSTAGMOGRAPHY AND TRANSCRANIAL DOPPLER IN EVALUATION OF PATIENTS WITH VERTIGO

T.A. Rageh, E.S. Mohamed, N.F. Kamel, A.M. Elattar

Assiut University Hospital, Assiut, Egypt

Introduction: Vertigo entails many adverse effects on social, emotional and occupational aspects of life. In a subset of patients vertigo may be a harbinger of vertebra-basilar stroke. This study was done to investigate the correlation of brainstem auditory evoked potentials (BAEP), electronystagmography (ENG) studies and results of transcranial Doppler (TCD) in patients with vertigo.

Methods: Twenty five patients suffering from vertigo aged 40-65 years and ten control persons with no history of vertigo were studied. The patients were subjected to clinical neuropsychiatric evaluation, basic audiological assessment, BAEP, ENG and TCD. Patients were divided into 2 groups depending on history, neurological examination and laboratory investigations. Group A includes 11 patients with vertebra-basilar insufficiency (VBI) and group B includes 14 patients with no manifestation of vertebra-basilar insufficiency (non-VBI)

Results: Our results revealed that ENG was abnormal in $81.8 \%$,TCD in $72.7 \%$ and BAEP in $63.6 \%$ of patients of VBI group. While in non-VBI group, the ENG was abnormal in $64.2 \%$, TCD in $21.4 \%$ and BAEP in $14.2 \%$ of patients. In comparing both groups, it was found that all three tests were abnormal in $63 \%$ of patients with VBI. While in the non-VBI group, all three tests were abnormal in $0 \%$ of patients. A significant correlation was found between TCD abnormalities and BAEP and ENG findings respectively in VBI group, and this correlation wasn't found in the other group.

Conclusion: Transcranial Doppler combined with BAEP and ENG can lead to early recognition of patients at risk of vertebrobasilar stroke. Hence, appropriate investigation and treatment could be done to prevent this outcome. 
EMULATING MULTICENTRE CLINICAL STROKE TRIALS: A NEW PARADIGM FOR STUDYING NOVEL INTERVENTIONS IN EXPERIMENTAL MODELS OF STROKE

P.M.W. Bath ${ }^{1}$, M. Macleod ${ }^{2}$, A.R. Green ${ }^{1}$

${ }^{1}$ University of Nottingham, Nottingham, United Kingdom; ${ }^{2}$ University of

Edinburgh, Edinburgh, United Kingdom

Background: Meta-analyses of NXY-059 in experimental stroke indicated neuroprotective activity. However, the failure of translation of it and other compounds suggests that new approaches to drug discovery in stroke are required.

Methods: We suggest that a fundamental paradigm shift is needed so that studies using animal models of stroke are conducted with the same degree of rigour, organisation and audit as human clinical trials. Individual laboratories should no longer perform preclinical studies independently; rather, coordinated groups of laboratories should work together in a manner similar to multicentre clinical trials. A central coordinating centre would manage study design, supply drug and placebo, and provide randomisation, central adjudication of outcomes (central blinded reading of lesion volume, histology, imaging, video of function), analysis and interpretation. The internal validity of studies would be ensured by using randomisation, concealment of allocation, and blinded surgery and outcome assessment; publication bias would be avoided by prior central registration of studies and a requirement to publish all completed data. A commitment to sample size calculations and the probable increased heterogeneity between centres makes it likely that studies will need to include larger numbers of animals. However, as with clinical trials, the regular monitoring of accruing data would allow studies to be discontinued early if there was clear evidence of efficacy, harm or futility. The additional costs involved should be seen as reasonable when compared to the resources that would have been expended in running large clinical trials that shows an intervention to be ineffective.

Conclusion: A major paradigm shift is needed in the design and delivery of preclinical stroke studies of novel interventions. Future studies should be performed in an analogous way to that used with multicentre clinical trials, with multiple laboratories collaborating through a central coordinating centre. Major sources of bias should be avoided at all cost. An ongoing multicentre study is testing this hypothesis.

\section{Experimental studies}

"STROKE IN SILICO": A COMPUTATIONAL APPROACH OF ACUTE STRO CLUES TO THE DIFFERENCE IN EFFICACY OF TREATMENTS BETWEEN ACUTE ISCHEMIC STROKE IN RODENTS AND HUMANS

M. Hommel ${ }^{1}$, M.A. Dronne ${ }^{2}$, E. Grenier ${ }^{3}$, G. Chapuisat $^{3}$, T. Dumont ${ }^{2}$,

A. Jaillard ${ }^{4}$, J.P. Boissel ${ }^{2}$

${ }^{1}$ INSERM CIC 003, Grenoble, France; ${ }^{2}$ IM Th UMR CNRS, Lyon, France;

${ }^{3}$ ENS UMR CNRS, Lyon, France; ${ }^{4}$ IFR1 INSERM, Grenoble, France

Background: Numeric modelling is a multidimentional and multiscales approach of complex phenomena and of their connections. Results can be expressed either in term of quantities or as a global description of a phenomenon. Numeric modelling can be applied in biology at different levels from cell receptors to populations and in different time frames from acute stroke to society outcomes. Moreover it can be useful to test new hypothesis. In therapy, one of its main applications is a faster and cheaper evaluation of therapies as compared to real world experimentations in animals or in human clinical trials. Our goal was to study differences in efficacy of neuroprotective agents between rodents and humans.

Methods: We used a description at the cellular and ionic levels of all the reported phenomena supposed to explain the difference in efficacy between human and rodents such as severity of ischemia and astrocytes/neurons ratio. Judgement criteria were imaging results (ADC) and energy metabolism (proportion of residual ATP). We used a series of simple differential equations for this approach.

We used a "global" approach (phenomenological) to study the effect of brain architecture (gyrocephalia). The end point was the development of spreading depression waves. For that characterisation we used diffusion equations. Computations were obtained using Matlab.

Results: We found differences in efficacy for various neuroprotective agents according to the difference in the ratio astrocytes/neurones and according to severity of ischemia.

Moreover, the spreading depression waves, "spontaneously developing" in ischemic brains were limited in their extension in gyrocephalic brains.

Conclusions: Our results may contribute to the explanation of a difference in efficacy of neuroprotective agents between rodents and humans, which are linked to differences in cells types" ratio and brain anatomy.
45 Experimental studies

THE PLASMA PROTEOME IN ISCHAEMIC STROKE: A CASE CONTROL STUDY

W.N. Whiteley ${ }^{1}$, Y.V. Berezovskaya ${ }^{2}$, D.J. Clarke ${ }^{2}$, P.A.G. Sandercock ${ }^{1}$, N. Anderson ${ }^{3}$, C.L. Mackay ${ }^{2}$

${ }^{1}$ Division of Clinical Neurosciences, Western General Hospital, University of Edinburgh, Edinburgh, United Kingdom; ${ }^{2}$ SIRCAMS, School of Chemistry, University of Edinburgh, Edinburgh, United Kingdom; ${ }^{3}$ Division of Community Health Sciences, University of Edinburgh, Edinburgh, United Kingdom

Background: There is no single blood marker that is diagnostic of ischaemic stroke. A candidate approach to selection of stroke markers has yet to be successful. We hypothesised that comparing the plasma proteomes of patients with and without ischaemic stroke might discover new diagnostic proteins.

Methods: The plasma of 8 patients ( 4 ischaemic stroke and 4 age, sex and severity matched ischaemic stroke mimics) was drawn within 24 hours of symptom onset and frozen at $-800^{\circ} \mathrm{C}$. Each sample was purified with a multiple affinity column to remove albumin, IgG, IgA, transferrin and antitrypsin. We investigated each sample blinded to stroke status with: (a) 500 micrometre monolithic liquid chromatography (LC) columns, coupled to a 12 Tesla Fourier-transform ion cyclotron resonance mass spectrometer (MS) 3 times (for quantitative measurement of individual protein species) and (b) tryptic digestion was made of each sample processed through LC columns and a high capacity trap MS 5 times (allowing more accurate identification of individual proteins). Data was processed with MS-Xelerator software. All analysis was blind to ischaemic stroke status.

Results: Proteins from previously published proteomic studies (apoprotein $\mathrm{C} 1$ and CIII) did not discriminate between stroke and non stroke samples. Across the 8 samples we identified 143 proteins. There was no statistically significant difference in levels of 86 proteins founding more than one sample between patients with ischaemic stroke and ischaemic stroke mimics (Welch modified t-test, level for significant difference $\mathrm{p}=0.0028$ ). Principle component analysis did not discriminate between stroke and non stroke samples.

Conclusions: The methods of mass spectrometry and statistical analysis of plasma samples for the discovery of diagnostic moieties need to be refined. They have yet to discover new diagnostic proteins. A larger sample size, and therefore multicentre collaboration, is needed to draw robust conclusions.

\section{Experimental studies}

\section{HEMISPHERE-SPECIFIC EFFECTS OF NOOTROPIC/VASOACTIVE DRUGS ON EMOTIONAL-MNESTIC FUNCTIONS AND CEREBRAL} HEMODYNAMICS IN STROKE PATIENTS

S.M. Kuznetsova, V.V. Kuznetsov, D.V. Shulzhenko, M.I. Tourta

Gerontology Institute AMS of Ukraine, Kiev, Ukraina

Background: Effects of nootropic/vasoactive drugs on emotional-mnestic processes and cerebral hemodynamics in ischemic stroke patients in early rehabilitation.

Methods: 215 elderly patients with atherothrombotic ischemic stroke in the carotic vessels ( 6 months after stroke), aged $67.3 \pm 2.8$ years, were examined before and after course treatment with the following drugs: GABA derivatives (Noofen, one tablet 3 times daily during one month; Piracetam, $200 \mathrm{mg}$, 3 times daily during one month); Glycin, $0.2 \mathrm{~g}, 3$ times daily during one month; Cortexin, $10 \mathrm{mg}$ intramuscularly, once a day during 10 days; Mexidol, $0.125 \mathrm{~g} 3$ times daily during one month; Instenon, $2.0 \mathrm{ml}$ intramuscularly, once a day, during 10 days, then 1 tablet 3 times daily during two months. Depressivity level was assessed by the gerontological scale, memory by memorization of 10 words, using the scale MMSE (Mini-Mental State Examination). Cerebral hemodynamics was determined by ultrasound duplex scanning on EN VISOR (Philips).

Results: With the right-hemispheric stroke, there was statistically reduced depressivity level under effects of noofen, piracetam, cortexin, glycin, and instenon; memory was activized under effects of glycin, cortexin, mexidol and instenon. Improvement of hemodynamics in the damaged hemisphere occurred owing to noofen and tyocetam action, in the intact hemisphere owing to instenon action. With the left-sided stroke, there was an activation of mnestic functions under effects of noofen, piracetam, cortexin, mexidol and instenon. The type of changes in cerebral hemodynamics was characterized by increase of velocity indices and decrease of peripheral resistance in the carotid and vertebro-basillar vessels under effects of glycin in the damaged hemisphere and under effects of noofen and piracetam in the intact hemisphere.

Conclusion: In the prescription of vasoactive and nootropic drugs to the patients with an ischemic stroke, it is recommended to consider hemispheric specificities of action of these drugs, thereby making more effective the process of recovery of emotional-mnestic activity of the brain and cerebral circulation. 
PERFUSION COMPUTED TOMOGRAPHY FOR ACUTE STROKE - A MODEL FOR EXPERIMENTAL VALIDATION

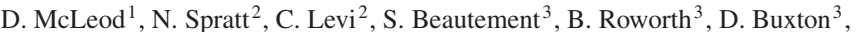
C. $\mathrm{Abel}^{3}, \mathrm{M}$. Calford ${ }^{3}, \mathrm{M}$. Parsons ${ }^{4}$

${ }^{1}$ School of Biomedical Sciences, University of Newcastle, Hunter Medical Research Institute, Newcastle, Australia; ${ }^{2}$ School of Biomedical Sciences, University of Newcastle, Hunter Medical Research Institute, Hunter New England Area Health Service, Newcastle, Australia; ${ }^{3}$ Hunter Health Imaging, Newcastle, NSW, Newcastle, Australia; ${ }^{4}$ Hunter Medical Research Institute, Hunter New England Area Health Service, Newcastle, Australia

Background: Computed Tomography Perfusion (CTP) imaging of brain poststroke may allow differentiation of potentially salvageable ("penumbra") and irreversibly injured ("infarct core") tissue. The technique is currently being widely applied in clinical stroke imaging. Validation of CTP parameters in the most commonly used experimental stroke model has not previously been undertaken. Experimental models, in which accurate timing of blood vessel occlusion and reperfusion are known, will allow detailed correlation of imaging parameters with final histology. The aim of these initial studies was to perform CTP imaging in rats with experimental stroke, to determine whether images of sufficient spatial resolution could be obtained to correlate with 24 hour histology.

Methods: Stroke was induced in male Wistar rats $(n=13)$ by the middle cerebral artery thread-occlusion method and a jugular venous line was inserted. CT scans were obtained on a 64-slice helical CT using a dynamic cerebral perfusion scanning method. Scans were obtained immediately pre- and post-occlusion and hourly for 3 hours. Histological changes of infarction were assessed after 24 hours.

Results: After optimisation of the perfusion and scanning parameters, colour maps were generated for cerebral perfusion (TTP, MTT), flow (CBF) and blood volume $(\mathrm{CBV})$ at multiple coronal planes. The ischaemic region was clearly demarcated on cerebral perfusion maps, and progressive reduction in the CBV within the centre of this region was seen over time. Imaging immediately after stroke onset allowed demonstration that $\mathrm{CBV}$ changes were biphasic in nature, with an initial decline, subsequent rise, and secondary decline.

Conclusions: This study has demonstrated for the first time, the feasibility of performing CTP in the most commonly used animal model of stroke. Definitive studies to determine optimal thresholds and reliability of CTP measures for infarct core and penumbra are in progress.

\section{Genetic disorders}

\section{Genetic disorders}

ABERRANT SPLICING DUE TO A SILENT NUCLEOTIDE CHANGE IN CCM2 GENE IN A FAMILY WITH CEREBRAL CAVERNOUS MALFORMATION S. Lanfranconi ${ }^{1}$, A. Bersano ${ }^{1}$, A. Tonelli ${ }^{2}$, S. Corti ${ }^{1}$, M.T. Bassi ${ }^{2}$, N. Bresolin ${ }^{1}$ ${ }^{1}$ Fondazione IRCCS Ospedale Maggiore Policlinico, Mangiagalli e Regina Elena, Milan, Italy; ${ }^{2}$ Istituto Scientifico E Medea, Bosisio Parini, Italy

Background: Cerebral cavernous malformations (CCMs), which account for 10 to $20 \%$ of the cerebral vascular lesions, can present either as sporadic or familial forms. Causal mutations have been identified in three genes: CCM1 (KRT1), CCM2 (MGC4607) or CCM3 (PDCD10). Here we describe a two-generations Italian family with four members showing multiple CCMs with variable locations and recurrent brainstem involvement.

Methods: A detailed familiar history was recorded. The family included 4 members showing CCMs: the proband, a 58 y.o male patient, his 24 y.o daughter and the proband's 64 y.o. brother with his 36 y.o son. After obtaining written informed consent we purified DNA from blood of all affected individuals and some healthy relatives. Mutation analysis was performed by direct sequencing of the PCR products. Genomic DNA from 130 unrelated healthy white Italian individuals was available as a control group.

Results: All 4 patients presented multiple supra and infratentorial cerebral angiomas, three showed also spinal cavernous angiomas and two presented cutaneous angiomas. Only two patients had a symptomatic presentation with neurological deficits due to hemorrhagic transformation of CCMs. Genetic analysis revealed a novel heterozygous CCM2 silent mutation (c.288G $>$ A) in exon 3 affecting the splicing and leading to the production of two different transcripts, one with skipping of exon 3 and the other of exons 3 and 4.

Conclusions: We report a two-generations family with four members showing CCMs lesions. The incomplete clinical penetrance in our family can be partly be explained by younger age of asymptomatic individuals. The putative protein products generated shows deletion of partly overlapping portion of the functional domain of CCM2 protein product, malcavernin. In addition, the out of frame deletion of both exons 3 and 4 lead to a premature protein truncation confirming that the loss-of-function is as a common mechanism underlying CCM2 mutations.

\section{Genetic disorders}

\section{NO EVIDENCE FOR A POTENTIAL ROLE OF MUTATIONS IN THE ACTA2} GENE IN THE ETIOLOGY OF CERVICAL ARTERY DISSECTIONS AND THORACIC AORTIC ANEURYSMS

T. Wiest ${ }^{1}$, C. Grond-Ginsbach ${ }^{1}$, I. Werner ${ }^{1}$, D. Kotelis ${ }^{2}$, P. Geisbuesch ${ }^{2}$, D. Boeckler ${ }^{2}$

${ }^{1}$ Department of Neurology, University of Heidelberg, Heidelberg, Germany; ${ }^{2}$ Department of Vascular and Endovascular Surgery, University of Heidelberg, Heidelberg, Germany

Background: A recent study suggested that missense mutations in the actin gene ACTA2 are responsible for $14 \%$ of inherited ascending thoracic aortic aneurysms and dissections. In the present study we performed a mutational analysis of the ACTA2 gene in a series of patients with spontaneous cervical artery dissections (sCAD) as well as in patients with thoracic aortic aneurysms (TAA).

Methods: 71 MRI-confirmed SCAD patients ( 22 women and 49 men, mean age $43,3 \pm 9,8$ years) and 37 TAA patients (treated by surgery; 9 women and 28 men, mean age $60,0 \pm 12,7$ years) were studied. From all patients genomic DNA fragments were amplified with intron-based, exon-specific primers of the exons 2 to 9 of the ACTA2. We searched for mutations in the coding region of the ACTA2 gene by single strand conformational polymorphism analysis and nucleotide sequencing analysis.

Results: Sequencing analysis revealed only one not yet described missense mutation in exon 9 in one sCAD patient (I371T). In 6/71 sCAD patients and 3/37 TAA patients we found a known single-nucleotide polymorphism (SNP) in the non-coding intron sequence of exon 5. In 1TAA patient we detected a heterozygous missense mutation in the intron sequence of exon 6 and in an nother TAA patient a heterozygous mutation in exon 8 . We did not find any mutation in exons 2, 3, 4 and 7 . Conclusion: In our series of SCAD patients and TAA patients we found one missense mutation in ACTA2. Our data suggest that mutations in the ACTA2 gene do not play an important role in the etiology of sCAD and TAA.

\section{Genetic disorders}

THE CONNECTIVE TISSUE PHENOTYPE OF PATIENTS WITH CERVICAL ARTERY DISSECTION (CAD) - GENETIC ANALYSIS OF LOCUS 15Q2 C. Grond-Ginsbach ${ }^{1}$, P. Lyrer ${ }^{2}$, T. Wiest ${ }^{1}$, R. Pjontek ${ }^{1}$, F. Fluri ${ }^{2}$, B. Chen ${ }^{3}$, B. Burwinkel ${ }^{3}$, T. Brandt ${ }^{4}$, I. Hausser ${ }^{5}$, S. Engelter ${ }^{2}$

${ }^{1}$ Department of Neurology, University of Heidelberg, Heidelberg, Germany; ${ }^{2}$ Department of Neurology, University of Basel, Basel, Switzerland; ${ }^{3}$ German Cancer Research Center, Heidelberg, Germany; ${ }^{4}$ Kliniken Schmieder, Heidelberg, Germany; ${ }^{5}$ Department of Dermatology, University of Heidelberg, Heidelberg, Germany

Background: Many patients with cervical artery dissection (CAD) show inherited abnormalities in their dermal connective tissue that can be identified by electron microscopy. Linkage analysis revealed a putative candidate locus for this connective tissue phenotype on chromosome $15 \mathrm{q} 2$. In this study we performed a high resolution genetic association study of this candidate locus.

Methods: 50 DNA samples from patients with CAD with electron-microscopyconfirmed connective tissue alterations were analyzed with Affymetrix hybrid arrays (6.0). The results were compared with findings in 25 CAD patients without electron microscopic connective tissue alterations as well as with findings from 500 healthy German control subjects. We evaluated SNPs that are located in the candidate locus (chromosome 15: 68-77 Mb).

Results: After filtering (SNPs with missing genotypes, with low information content, or with genotype distribution out of Hardy Weinberg equilibrium were not analyzed) we studied 1436 SNPs within the candidate locus and compared genotypes in patients and controls. Significant assocations $(p=0.0026)$ were observed for four SNPs within the gene encoding thrombospondin type I domain containing 4 (THSD4).

Conclusion: The connective tissue phenotype associated with CAD might be related to genetic variation in the THBS4 gene. This gene is located within the candidate region on chromosome $15 \mathrm{q}$ and showed strongest association with the connective tissue phenotype. We speculate that copy number variants $(\mathrm{CNV}$, small deletions and duplications) within the THBS4 gene might be in tight linkage disequilibrium with the associated SNP alleles and aim at further CNV analyses with the aid of sophisticated biometrical and statistical software. 
SUGGESTIVE CLINICAL FEATURES AND NOTCH3 GENE VARIANTS: CADASIL OR CADASIL-LIKE?

A. Mendes ${ }^{1}$, S. Ferreira ${ }^{2}$, R. Fonseca ${ }^{3}$, M. Carvalho ${ }^{1}$, C. Garrett ${ }^{1}$, E. Azevedo ${ }^{1}$ ${ }^{1}$ Neurology Department of Hospital de S. João and Faculty of Medicine of Porto, Porto, Portugal $;{ }^{2}$ Genetics Department of Faculty of Medicine of Porto, Porto, Portugal; ${ }^{3}$ Neurology Department of Hospital de S. João, Porto, Portugal

Background: Hereditary forms of cerebrovascular disease are relatively rare. CADASIL is the most frequently reported. The genetic diagnosis is confirmed when NOTCH3 gene mutations affecting cystein residues are found. We present a family case with a CADASIL-like syndrome, where genetics were not conclusive. Our aim is to discuss if our patients have CADASIL or a non-characterized hereditary small vessel brain disease as reported before. Familiar case report Index 1- 45-year-old woman, smoker, history of visual aura episodes, mnesic complaints, executive dysfunction and an episode of left hemiparesis. Sister 247-year-old woman, controlled arterial hypertension, progressive cognitive decline in last 3 years and a middle cerebral artery stroke 2 years ago. 3 - Their mother had also a dementia, but died meanwhile, not finishing investigation. Both sisters did investigation for young adult causes of stroke, including immunologic and prothrombotic studies, that were negative. Cervical and transcranial ultrasound and echocardiography were normal. Brain MRI in all 3 women revelead ischaemic lesions in fronto-parietal white matter, temporal region, basal ganglia and pons. First patient also had external capsule lesions and a negative skin biopsy regarding osmiophilic deposits. Sequencing analyses of exons 2-23 of the NOTCH3 gene in both sisters presented the following sequence variants: p.A1020P in homozigoty (exon 19), p.S497L (exon 9) and p.V1183M (exon 22).

Discussion: Clinical picture and neuroimaging are suggestive of CADASIL. However, genetic study did not find a classical pathological mutation. We point out if the p.A1020P, already considered a polimorfism in portuguese population, can be pathogenic in homozigoty or if the referred 3 sequence variants together produce an abnormal protein causative of the disease. Excluding these possibilities, this family could be affected by another small vessel disease yet to be defined.

\section{Genetic disorders}

\section{POLYGENIC JUVENILE STROKE}

A. Bersano $^{1}$, E. Ballabio ${ }^{1}$, A. Pezzini ${ }^{2}$, A. Padovani ${ }^{2}$, N. Bresolin ${ }^{1}$, P. Baron ${ }^{1}$, L. Candelise ${ }^{1}$

${ }^{1}$ Fondazione IRCCS Ospedale Maggiore Policlinico, Mangiagalli e Regina Elena, Milan, Italy; ${ }^{2}$ Neurologia Vascolare, Spedali Civili di Brescia, Brescia, Italy

Background: The prevalence of ischemic stroke affecting patients younger than 50 y.o ranges from 3 to $12.3 \%$. Epidemiological and familial studies provided evidences that genetic factors have a role in stroke occurrence particularly among individuals presenting with stroke at young age. Monogenic disorders explain less than $1 \%$ of all young strokes. Stroke is a complex disease in which genetic factors have been supposed to act in additive co-effect. However, despite family history studies demonstrated that genetic factors are much more important in young individuals, most of studies conducted so far did not include age stratification analyses. The aim of this paper is to identify, thought an updated literature review, the role of several candidate genes in young adult stroke.

Methods: Paper search was undertaken in electronic databases from 1960 to May 2008 for case control studies, prospective cohort studies, reviews and meta-analyses in English-language investigating the role of several candidate genes (F5, F2, FGA, FGB, F7, F12, F13A1, vWF, SERPINE1, ITGB3, ITGA2B, ITGA2, GP1BA, ACE, AGT, NOS3, MTHFR, CBS, MTR, APOE, LPL, PON1, PDE4D, ALOX5AP) in ischemic stroke in patients aged from 0 to 50 years. The results of association studies were reported as odds ratio (OR) and $95 \%$ confidence intervals (95\% CI) as calculated by the authors.

Results: we found that only three polymorphisms (c.1691G $>$ A, c.20210G $>$ A, c. $677 \mathrm{C}>\mathrm{T}$ ) of three candidate genes (F5, F2, MTHFR) are possibly associated with increased stroke risk in young subjects.

Discussion: Although we did not identify significant associations between juvenile stroke and specific genes, we indicate a list of genes possibly generating a susceptibility to stroke, that could be a starting point for future research. The hope is that understanding the genetic basis of juvenile stroke will lead to better management of these patients and will facilitate the understanding of pathogenetic mechanisms of adult stroke.
6 Genetic disorders

ISCHEMIC STROKE AND PATENT FORAMEN OVALE, RISKFACTORS AND GENETIC PROFILE

M. Lantz, C. Sjöstrand, K. Kostulas

Dept. Neurology, R52, Karolinska University Hospital, Karolinska Institutet, Stockholm, Sweden

Background: Patent foramen ovale (PFO) is considered being a risk factor for ischemic cerebrovascular disease (ICVD), especially in young people. However its roll is controverial and needs further investigation. The aim of this report was to study konventional risk factors as well as the distribution of 100 polymorphisms in 47 suspected susceptibility genes for ICVD in stokepatients with or without a PFO. Methods: In the South Stockholm Ischemic Stroke Study (SSISS) 928 ICVD patients and 602 controls were genotyped for 100 different gene polymorphisms. The strokepatients also underwent relevant investigation and standardized blood tests. Patients that, as part of their investigation, underwent a transeosophageal echocardiography were divided into having a PFO or not.

Results: There were no significant differences in the two groups when looking at konventional risk factors or blood analysis. Three different polymorphisms located in the pro-thrombin (F2) and apolipoprotein-C III (APO-CIII) genes were significantly associated with ICVD and PFO. The strongest association being for F2 ( $\mathrm{p}=0.0049$, OR 26.429)

Conclusions: We found that F2, which previously has been described as being a possible link between PFO and ICVD, were significantly associated with ICVD and PFO. Two other polymorphisms in the APO-CIII gene were weakly associated to PFO and ICVD.

\section{Genetic disorders}

\section{LEUKOCYTOSIS IN PATIENTS WITH ACUTE CERVICAL ARTERY} DISSECTION IS ASSOCIATED WITH VARIATION IN THE SELENOPROTEIN S GENE (SEPS1)

S.S. Aksay ${ }^{1}$, S. Rose ${ }^{2}$, F. Fluri ${ }^{3}$, M. Kloss ${ }^{1}$, A. Pezzini ${ }^{4}$, E. del Zotto ${ }^{4}$, A. Padovani ${ }^{4}$, M.-L. Arnold ${ }^{1}$, I. Werner ${ }^{1}$, P. Lyrer ${ }^{3}$, C. Lichy ${ }^{1}$, S. Engelter ${ }^{3}$, A.J. Grau ${ }^{2}$, C. Grond-Ginsbach ${ }^{1}$

${ }^{1}$ Department of Neurology, University of Heidelberg, Heidelberg, Germany; ${ }^{2}$ Deparment of Neurology, Klinikum der Stadt Ludwigshafen a.Rh., Ludwigshafen, Germany; ${ }^{3}$ Department of Neurology, University of Basel, Basel, Switzerland; ${ }^{4}$ Department of Neurology, University of Brescia, Brescia, Italy

Background: Patients with cerebrovascular events might develop a reactive leukocytosis as part of the acute peripheral inflammatory response. In patients with acute cervical artery dissection (CAD) we sought to identify whether pro-inflammatory gene variants contribute to this reactive leukocytosis.

Methods: Leukocyte number and common pro-inflammatory genetic variants of interleukin 1 receptor antagonist (IL1RN), interleukin 6 (IL6), tumor necrosis factor alpha (TNF) and selenoprotein S (SEPS1) were analyzed in 158 patients with acute CAD from the Upper Rhine Valley region.

Results: Leukocyte counts $>11.000 / \mu 1$ were found in 47 patients $(30 \%)$ during acute CAD. Leukocyte counts were positively correlated with stroke severity (NIHSS at admission) and negatively correlated with the latency between onset of CAD symptoms and blood testing. Elevated leukocyte counts were associated $(\mathrm{p}=0.013)$ with a pro-inflammatory variant of SEPS1 (A-allele of rs28665122), but not with variants of other inflammatory genes. In multiple regression analysis the pro-inflammatory SEPS1 variant remained an independent determinant of leukocyte count $(\mathrm{p}=0.005)$.

Conclusions: These findings demonstrate that the pro-inflammatory promoter variant of SEPS1 is associated with leukocytosis during acute CAD. A similar association between SEPS1 and leukocytosis was found in an independent series of 64 patients with acute CAD from Northern Italy and in a cohort of common stroke patients from Ludwigshafen (Germany).This latter observation suggests that the association between SEPS1 and leukocytosis is also found in a mixed stroke population. 